\title{
Generation of balanced viscosity solutions to rate-independent systems via variational convergence
}

\author{
Giovanni A. Bonaschi ${ }^{1,2} \cdot$ Riccarda Rossi $^{3}$ (D) \\ Received: 15 October 2018 / Accepted: 4 May 2019 / Published online: 28 May 2019 \\ (c) Fondazione Annali di Matematica Pura ed Applicata and Springer-Verlag GmbH Germany, part of Springer \\ Nature 2019
}

\begin{abstract}
In this paper, we investigate the origin of the balanced viscosity solution concept for rateindependent evolution, in the setting of a finite-dimensional space. Namely, given a family of dissipation potentials $\left(\Psi_{n}\right)_{n \in \mathbb{N}}$ with superlinear growth at infinity and suitably converging to a 1-positively homogeneous potential $\Psi_{0}$, and a smooth energy functional $\mathcal{E}$, we provide sufficient conditions on them ensuring that the solutions of the associated (generalized) gradient systems $\left(\Psi_{n}, \mathcal{E}\right)$ converge as $n \rightarrow \infty$ to a Balanced Viscosity solution of the rate-independent system driven by $\Psi_{0}$ and $\mathcal{E}$. In specific cases, we also obtain results on the reverse approximation of balanced viscosity solutions by means of solutions to gradient systems. Our approach is based on the key observation that solutions to gradient systems/that Balanced Viscosity solutions to rate-independent systems can be characterized as (null-)minimizers of suitable trajectory functionals, for which we indeed prove MosCO-convergence. As particular cases, our analysis encompasses both the vanishing-viscosity approximation of rate-independent systems from Mielke et al. (ESAIM Control Optim Calc Var 18(1):36-80, 2012, J Eur Math Soc 18(9):2107-2165, 2016), and their stochastic derivation developed in Bonaschi and Peletier (Contin Mech Thermodyn 28:1191-1219, 2016).
\end{abstract}

Keywords Gradient systems · Rate-independent systems · Balanced viscosity solutions · Vanishing viscosity $\cdot$ Large deviations $\cdot$ Variational convergence

Mathematics Subject Classification Primary 35A15 · Secondary 34A60

G.A.B. kindly acknowledges support from the Nederlandse Organisatie voor Wetenschappelijk Onderzoek (NWO), VICI Grant 639.033.008.

R.R. has been partially supported by GNAMPA (Gruppo Nazionale per l'Analisi Matematica, la Probabilità e le loro Applicazioni) of INdAM (Istituto Nazionale di Alta Matematica).

$\bowtie$ Riccarda Rossi

riccarda.rossi@unibs.it

Giovanni A. Bonaschi

giovanni.bonaschi@gmail.com

1 Technische Universiteit Eindhoven, 5600 MB Eindhoven, The Netherlands

2 AVIVA, Milan, Italy

3 DIMI, Università di Brescia, via Branze 38, 25133 Brescia, Italy 


\section{Introduction}

Over the last years, rate-independent systems have been the object of intensive mathematical investigations. This is undoubtedly due to their vast range of applicability. Indeed, this kind of processes seems to be ubiquitous in continuum mechanics, ranging from shape-memory alloys to crack propagation, from elastoplasticity to damage and delamination. They also occur in fields such as ferromagnetism and ferroelectricity. We refer to [32,39] for a thorough survey of all these problems.

Besides its applicative relevance, though, rate-independent evolution has an own, intrinsic, mathematical interest. This is apparent already in the context of a finite-dimensional rateindependent system, to which we shall confine the analysis developed within this paper. In general, such a system is driven by a dissipation potential $\Psi_{0}: \mathbb{R}^{d} \rightarrow[0,+\infty$ ) (nondegenerate), convex, and positively homogeneous of degree 1 , and an energy functional $\mathcal{E}:[0, T] \times \mathbb{R}^{d} \rightarrow \mathbb{R}$; in particular, throughout the paper, we will consider a smooth energy $\mathcal{E}$ such that the power function $\partial_{t} \mathcal{E}$ is controlled by $\mathcal{E}$ itself, namely

$$
\begin{aligned}
& \mathcal{E} \in \mathrm{C}^{1}\left([0, T] \times \mathbb{R}^{d}\right) \quad \text { and } \\
& \exists C_{1}, C_{2}>0 \forall(t, u) \in[0, T] \times \mathbb{R}^{d}: \quad\left|\partial_{t} \mathcal{E}(t, u)\right| \leq C_{1} \mathcal{E}(t, u)+C_{2} .
\end{aligned}
$$

The pair $\left(\Psi_{0}, \mathcal{E}\right)$ gives rise to the simplest example of rate-independent evolution, namely the gradient system

$$
\partial \Psi_{0}\left(u^{\prime}(t)\right)+\mathrm{D} \mathcal{E}(t, u(t)) \ni 0 \quad \text { for a.a. } t \in(0, T),
$$

where $\partial \Psi_{0}: \mathbb{R}^{d} \rightrightarrows \mathbb{R}^{d}$ is the subdifferential of $\Psi_{0}$ in the sense of convex analysis, whereas $\mathrm{D} \mathcal{E}$ is the differential of the map $u \mapsto \mathcal{E}(t, u)$. Due to the 0 -homogeneity of $\partial \Psi_{0},(1.1)$ is invariant for time rescalings, i.e., it is rate-independent. Now, it is well known that, even in the case of a smooth energy $\mathcal{E}$, if $u \mapsto \mathcal{E}(t, u)$ fails to be strictly convex, then absolutely continuous solutions to (1.1) need not exist. In the last two decades, this has motivated the development of various weak solvability concepts for (1.1) and, in general, for rate-independent systems in infinite-dimensional Banach spaces, or even topological spaces.

While referring to $[33,39]$ for a survey of all weak notions of rate-independent evolution, in the following lines we shall focus on the concepts of energetic and balanced viscosity solutions. The study of these notions poses several interesting problems already in the finitedimensional context and motivates the analysis developed in this paper.

Energetic and Balanced Viscosity solutions to rate-independent systems. The concept of energetic solution was first proposed in [43] and fully analyzed in [44]; an analogous notion, referred to as quasistatic evolution, was in parallel developed within the realm of brittle fracture, cf. [19,22].

It consists of the global stability condition, holding at every $t \in[0, T]$,

$$
\mathcal{E}(t, u(t)) \leq \mathcal{E}(t, z)+\Psi_{0}(z-u(t)) \quad \text { for every } z \in \mathbb{R}^{d},
$$

and of the $\left(\Psi_{0}, \mathcal{E}\right)$-energy-dissipation balance

$$
\mathcal{E}(t, u(t))+\operatorname{Var}_{\Psi_{0}}(u ;[0, t])=\mathcal{E}(0, u(0))+\int_{0}^{t} \partial_{t} \mathcal{E}(s, u(s)) \mathrm{d} s \quad \text { for every } t \in[0, T] .
$$


Indeed, $\left(\mathrm{E}_{\Psi_{0}, \mathcal{E}}\right)$ involves the dissipated energy $\operatorname{Var}_{\Psi_{0}}(u ;[0, t])$ (where $\operatorname{Var}_{\Psi_{0}}$ denotes the notion of total variation induced by $\left.\Psi_{0}\right)$, the stored energy $\mathcal{E}(t, u(t))$ at the process time $t$, the initial energy $\mathcal{E}(0, u(0))$, and the work of the external forces. Since the energetic formulation $(\mathrm{S})-\left(\mathrm{E}_{\Psi_{0}, \mathcal{E}}\right)$ only features the (assumedly smooth) power of the external forces $\partial_{t} \mathcal{E}$, and no other derivatives, it is particularly suited to solutions with discontinuities in time. It is also considerably flexible and can be indeed given for rate-independent processes in general topological spaces, cf. [35]. That is why, it has been exploited in a great variety of applicative contexts, ranging from fracture, damage, and delamination to plasticity, shape-memory alloys, ferroelectricity, to name a few; we refer to [39] for a comprehensive survey.

Nonetheless, over the years it has become apparent that, in the very case of a nonconvex dependence $u \mapsto \mathcal{E}(t, u)$, the global stability (S) fails provide a truthful description of the system behavior at jumps, leading to solutions jumping 'too early' and 'too long' (i.e., into very far-apart energetic configurations), as shown for instance by the example [31, Ex.6.1], and by the characterization of energetic solutions to (one-dimensional) rate-independent systems in [48].

This circumstance has led to the introduction of alternative weak solvability concepts for (1.1) and its generalizations. In particular, [20] first set forth the vanishing-viscosity regularization of the rate-independent system as a selection criterion for mechanically feasible weak solutions. The vanishing-viscosity approach has in fact proved to be a robust method in diverse applications, ranging from plasticity (cf., e.g., [11,17,18]), to fracture (cf., e.g., [26,30,52]), and to damage (cf., e.g., [14,28]) models. The solution concept obtained through this approach has been codified under the name of balanced viscosity solution in [41,42]. We also refer to [46] for an alternative derivation of balanced viscosity solutions via time discretization, and to [36], where the notion was recovered with the so-called epsilon-neighborhood method.

Let us briefly illustrate the vanishing-viscosity approach: We "augment by viscosity" the dissipation potential $\Psi_{0}$ and thus introduce

$$
\Psi_{\varepsilon}(v):=\Psi_{0}(v)+\frac{\varepsilon}{2}\|v\|^{2},
$$

with $\|\cdot\|$ a second norm on $\mathbb{R}^{d}$, which may or may not coincide with the norm associated with $\Psi_{0}$. The corresponding (generalized) gradient system $\left(\Psi_{\varepsilon}, \mathcal{E}\right)$, namely the doubly nonlinear differential inclusion

$$
\partial \Psi_{\varepsilon}\left(u^{\prime}(t)\right)+\mathrm{D} \mathcal{E}(t, u(t)) \ni 0 \quad \text { for a.a. } t \in(0, T),
$$

thus provides an approximation of the rate-independent system (1.1). Since $\Psi_{\varepsilon}$ has superlinear growth at infinity, (1.3) does admit absolutely continuous solutions. It is to be expected that, as the viscosity parameter $\varepsilon$ vanishes, solutions $\left(u_{\varepsilon}\right)_{\varepsilon}$ to (1.3) will converge to a suitable weak solution to the rate-independent system (1.1). In [41], it was indeed shown that any limit curve $u \in \mathrm{BV}\left([0, T] ; \mathbb{R}^{d}\right)$ of the functions $\left(u_{\varepsilon}\right)_{\varepsilon}$ complies with the stability condition

$$
-\mathrm{DE}(t, u(t)) \in K^{*}:=\partial \Psi_{0}(0) \text { for a.a. } t \in(0, T),
$$

and with the energy-dissipation balance

$$
\operatorname{Var}_{\Psi_{0}, \mathfrak{p}, \mathcal{E}}(u ;[0, t])+\mathcal{E}(t, u(t))=\mathcal{E}(0, u(0))+\int_{0}^{t} \partial_{t} \mathcal{E}(s, u(s)) \mathrm{d} s \quad \text { for all } t \in[0, T],
$$


which individuate the notion of balanced viscosity solution to the rate-independent system $(\mathrm{S})$. Although $\left(\mathrm{S}_{\mathrm{loc}}\right)$ and $\left(\mathrm{E}_{\Psi_{0}, \mathfrak{p}, \mathcal{E}}\right)$ look similar to $(\mathrm{S})$ and $\left(\mathrm{E}_{\Psi_{0}, \mathcal{E}}\right)$, they are in fact significantly different. First of all, $\left(\mathrm{S}_{\mathrm{loc}}\right)$ is in fact a local version of the global stability (S). Secondly, $\left(\mathrm{E}_{\Psi_{0}, \mathfrak{p}, \mathcal{E}}\right)$ shares the same structure with the energy-dissipation balance $\left(\mathrm{E}_{\Psi_{0}, \mathcal{E}}\right)$, but it features

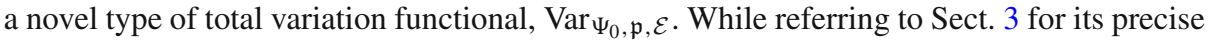
definition (cf. (3.23) ahead), we may mention here that, in addition to the dissipation potential $\Psi_{0}, \operatorname{Var}_{\Psi_{0}, \mathfrak{p}, \mathcal{E}}$ involves the vanishing-viscosity contact potential

$$
\mathfrak{p}(v, \xi):=\inf _{\varepsilon>0}\left(\Psi_{\varepsilon}(v)+\Psi_{\varepsilon}^{*}(\xi)\right)=\Psi_{0}(v)+\|v\| \min _{\zeta \in K^{*}}\|\xi-\zeta\|_{*},
$$

which indeed lies at the core of the balanced viscosity concept. The second equality in (1.4) ensues from a direct calculation; $K^{*}$ is the stable set from $\left(\mathrm{S}_{\mathrm{loc}}\right)$ and $\|\cdot\|_{*}$ the dual norm of the "viscosity-related" norm $\|\cdot\|$. As we will see in Sec. 3 with more detail, the functional $\mathfrak{p}$ thus encodes how viscosity, neglected in the vanishing-viscosity limit, pops back into the description of the solution behavior at jumps, whereas in the continuous ('sliding') regime, the system is only governed by the 1-homogeneous dissipation $\Psi_{0}$.

A characterization of balanced viscosity solutions, again for one-dimensional systems, has been provided in [48], showing that they model jumps more accurately than energetic solutions. On the other hand, as evident from (1.4), this notion seems to be strongly reminiscent of the vanishing-viscosity approximation (1.3).

It is thus natural to wonder whether there are ways, alternative to the vanishing viscosity $[41,42]$ and to the time-discretization [46] (cf. also [36]) approaches, to generate balanced viscosity solutions. The present paper aims to contribute in this direction.

The stochastic origin of balanced viscosity solutions. Recently, this question has been answered affirmatively in [12], investigating the role of stochasticity in the origin of rate independence, in the context of one-dimensional rate-independent systems (i.e., with (1.1) set in the ambient space $\mathbb{R}$ ). The motivation for the analysis carried out in [12] stems from the argument that rate independence may arise through the interplay between thermal noise and a rough energy landscape, cf. $[5,9,25,47]$. The approach to rate-independent evolution developed in [12] is akin to that in [1,2], where a connection has been established between the evolution of a class of many-particle systems and Wasserstein gradient flows, through a large-deviations principle. We also refer to [29,38], where deeper insight has been gained into the principles underlying this connection within the more general context of (generalized) gradient structures; in this mainstream, [12] has, however, been the first paper investigating rate-independent, in place of rate-dependent, evolution.

More specifically, [12] has focused on a continuous-time Markov jump process $t \mapsto X_{t}^{h}$ on a one-dimensional lattice, with lattice spacing $\frac{1}{h}, h \in \mathbb{N}$. While referring to Sect. 2 for more details, we may mention here that this process models the evolution of a Brownian particle in a wiggly energy landscape, involving the energy $\mathcal{E}$, in the following way. If the particle is at the position $x$ at time $t$, then it jumps in continuous time to its neighbors $x \pm \frac{1}{h}$ with rates $h r^{ \pm}(x)$, where $r^{ \pm}(x)=\alpha \exp (\mp \beta \mathrm{DE}(t, x))$. Here, $\alpha$ and $\beta$ are positive parameters, the former characterizing the rate of jumps, and thus the global timescale of the process, and the latter related to noise.

First of all, in [12] it was shown that the deterministic limit, in a 'large-deviations' sense, as $h \rightarrow \infty$ and for $\alpha$ and $\beta$ fixed, of this stochastic process solves

$$
u^{\prime}(t)=2 \alpha \sinh (-\beta \mathrm{DE}(t, u(t))) \quad \text { for a.a. } t \in(0, T) .
$$


Observe that the latter equation is a reformulation of the (generalized) gradient system

$$
\partial \Psi_{\alpha, \beta}\left(u^{\prime}(t)\right)+\mathrm{DE}(t, u(t)) \ni 0 \quad \text { for a.a. } t \in(0, T),
$$

where the convex dissipation potential $\Psi_{\alpha, \beta}$ is such that its Fenchel-Moreau convex conjugate fulfills $\partial \Psi_{\alpha, \beta}^{-1}(\xi)=\partial \Psi_{\alpha, \beta}^{*}(\xi)=\left\{\mathrm{D} \Psi_{\alpha, \beta}^{*}(\xi)\right\}=\{2 \alpha \sinh (\beta \xi)\}$. More precisely, in [12] it was proved that the process $X^{h}$ satisfies a large-deviations principle (cf. Sec. 2), with rate function given by the functional of trajectories $\tilde{J}_{\Psi_{\alpha, \beta}, \mathcal{E}}: \mathrm{BV}\left([0, T] ; \mathbb{R}^{d}\right) \rightarrow[0,+\infty]$ defined by

$$
\begin{aligned}
\tilde{\mathscr{J}}_{\Psi_{\alpha, \beta}, \mathcal{E}}(u):=\beta( & \int_{0}^{T}\left(\Psi_{\alpha, \beta}\left(u^{\prime}(t)\right)+\Psi_{\alpha, \beta}^{*}(-\mathrm{D} \mathcal{E}(t, u(t)))\right) \mathrm{d} t \\
& \left.+\mathcal{E}(T, u(T))-\mathcal{E}(0, u(0))-\int_{0}^{T} \partial_{t} \mathcal{E}(t, u(t)) \mathrm{d} t\right)
\end{aligned}
$$

if $u \in \mathrm{AC}\left([0, T] ; \mathbb{R}^{d}\right)$, and $+\infty$ else. In fact, the null-minimizers of $\tilde{\mathscr{J}}_{\Psi_{\alpha, \beta}, \mathcal{E}}$ are solutions to the gradient system (1.5), as shown in Lemma 3.1 ahead. Let us mention that this kind of argument, relating solutions of evolutionary equations to the (null-)minimization of functionals of entire trajectories, can be traced back to the seminal BRÉZIS- EKELAND- NAYROLES principle [7,8,45], cf. also [51], and the monograph [23] for a comprehensive overview.

Secondly, the variational limits of the functionals $\tilde{\mathscr{J}}_{\Psi_{\alpha, \beta}, \mathcal{E}}$ have been addressed in [12] under different scalings of the parameters $\alpha$ and $\beta$, leading to gradient flow or rateindependent evolution. To illustrate the result in the latter case, here and throughout the paper we will confine the discussion to the following choice of parameters: $\alpha=\alpha_{n}:=\frac{e^{-n A}}{2}$ and $\beta:=\beta_{n}=n$, with $n \in \mathbb{N}$. In this case, the associated dissipation potentials are given by

$$
\Psi_{n}(v):=\Psi_{\alpha_{n}, \beta_{n}}(v)=\frac{v}{n} \log \left(\frac{v+\sqrt{v^{2}+e^{-2 n A}}}{e^{-n A}}\right)-\frac{1}{n} \sqrt{v^{2}+e^{-2 n A}}+\frac{e^{-n A}}{n} .
$$

In [12, Thm. 4.2], it was proved that that the functionals of trajectories $\mathscr{J}_{\Psi_{n}, \mathcal{E}}:=\frac{1}{n} \tilde{\mathscr{J}}_{\Psi_{n}, \mathcal{E}}$ converge in the sense of MosCO with respect to the weak-strict topology in $\mathrm{BV}\left([0, T] ; \mathbb{R}^{d}\right)$, to the functional $\mathscr{J}_{\Psi_{0}, \mathfrak{p}, \mathcal{E}}: \mathrm{BV}\left([0, T] ; \mathbb{R}^{d}\right) \rightarrow[0,+\infty]$ defined by

$$
\begin{aligned}
\mathscr{J}_{\Psi_{0}, \mathfrak{p}, \mathcal{E}}(u):= & \operatorname{Var}_{\Psi_{0}, \mathfrak{p}, \mathcal{E}}(u ;[0, T])+\int_{0}^{T} I_{K^{*}}(-\mathrm{D} \mathcal{E}(t, u(t))) \mathrm{d} t \\
& +\mathcal{E}(T, u(T))-\mathcal{E}(0, u(0))-\int_{0}^{T} \partial_{t} \mathcal{E}(s, u(s)) \mathrm{d} s,
\end{aligned}
$$

with $\Psi_{0}(v)=A|v|, \mathfrak{p}$ given by (1.4) and the associated total variation functional $\operatorname{Var}_{\Psi_{0}, \mathfrak{p}, \mathcal{E}}$ defined in (3.23) ahead, and with $I_{K^{*}}$ denoting the indicator function of the set stable set $K^{*}=\partial \Psi_{0}(0)=[-A, A]$. While postponing the precise definition of MosCO-convergence in $\mathrm{BV}\left([0, T] ; \mathbb{R}^{d}\right)$ to $(3.17)$ ahead, let us only mention that $\mathrm{BV}\left([0, T] ; \mathbb{R}^{d}\right)$ is the appropriate space for the solutions to rate-independent systems, liable to jump as functions of time.

The Mosco-convergence result in [12, Thm. 4.2] establishes a deep connection between the generalized gradient system (1.5) and the one-dimensional rate-independent system driven by $\Psi_{0}(v)=A|v|$ and $\mathcal{E}$. In fact, (null-)minimizers of $\mathscr{J}_{\Psi_{0}, \mathfrak{p}, \mathcal{E}}$ are balanced viscosity solutions of the rate-independent system driven by $\Psi_{0}$ and $\mathcal{E}$ (cf. Proposition 3.7 ahead). Therefore, on the one hand the $\Gamma$-lim inf-estimate verified within Mosco-convergence, joint with the observation that (null-)minimizers of $\mathscr{J}_{\Psi_{n}, \mathcal{E}}$, are solutions to the gradient system (1.5), ensures the convergence of the latter to balanced viscosity solutions of the rate-independent system. On the other hand, the $\Gamma$-lim sup-estimate yields a reverse approximation result. 
Therefore, [12, Thm. 4.2] ultimately reveals the connection between the jump process $X^{h}$ and the (one-dimensional) rate-independent system (1.1), understood in a balanced viscosity sense. Furthermore, observe that the functionals $\Psi_{n}$ from (1.6) are not of form (1.2). Hence, this result provides a way, alternative to the vanishing viscosity approach, to generate balanced viscosity solutions.

\subsection{Our results}

Our first motivation for this work was to extend the 'stochastic generation' of balanced viscosity solutions unraveled

in [12] to the multi-dimensional rate-independent system (1.1), where now

$$
\Psi_{0}(v):=A\|v\|_{1} \quad \text { for all } v \in \mathbb{R}^{d}, \quad \text { with }\|v\|_{1}:=\sum_{i=1}^{d}\left|v_{i}\right| .
$$

The first obvious difference between the one- and the multi-dimensional contexts is that, even conjecturing that the viscosity contact potential defining the limiting Balanced Viscosity solution notion is of form (1.4), in this multi-dimensional context it is no longer obvious which choice of the viscous norm $\|\cdot\|$ should enter into (1.4). With our main results, Theorem 5.2 (lim inf-estimate) and Theorem 5.9 (lim sup-estimate), we have shown that the

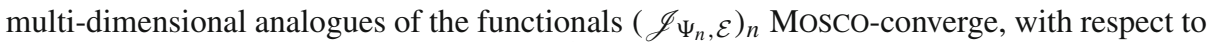
the weak-strict topology of $\mathrm{BV}\left([0, T] ; \mathbb{R}^{d}\right)$, to the functional $\mathscr{J}_{\Psi_{0}, \mathfrak{p}, \mathcal{E}}$ featuring the total variation functional associated with the contact potential

$$
\mathfrak{p}(v, \xi):=\|v\|_{1}\left(A \vee\|\xi\|_{\infty}\right) \quad \text { with }\|\xi\|_{\infty}:=\max _{i=1, \ldots, d}\left|\xi_{i}\right| .
$$

It can be checked that $\mathfrak{p}$ from (1.9) is indeed of form (1.4). In this case, however, the 'viscous' norm $\|\cdot\|$ in fact coincides with that associated with $\Psi_{0}$ from (1.8), i.e., $\|v\|=\|v\|_{1}$. Therefore, the notion of balanced viscosity solution arising from the stochastic approximation coincides with the one in which the 1-homogeneous dissipation potential $\Psi_{0}$ is perturbed by a (superlinear) function of $\Psi_{0}$ itself. Referring to this case, we shall speak of vanishing $\Psi_{0}$-viscosity, cf. Example 5.5 ahead.

Our $\Gamma$-lim inf Theorem 5.2 has in fact a broader and deeper scope and indeed contributes to understanding the origin of rate-independent evolution in a Balanced Viscosity sense. More precisely, in this paper

(1) We will introduce an 'extended' notion of Balanced Viscosity solution, induced by a class of viscosity contact potential $\mathrm{p}:[0,+\infty) \times \mathbb{R}^{d} \times \mathbb{R}^{d} \rightarrow[0+\infty], \mathrm{p}=\mathrm{p}(\tau, v, \xi)$, cf. Def. 3.2 ahead, in place of the vanishing-viscosity contact potential $\mathfrak{p}$ from, e.g., (1.4). We may understand the functional $p$ as obtained by augmenting $\mathfrak{p}$ with the time variable, in that the contact potentials $\mathfrak{p}(v, \xi)$ from (1.4), for instance, stem from setting $\tau=0$ in (specific choices of) $\mathrm{p}$, i.e., $\mathrm{p}(0, v, \xi)=\mathfrak{p}(v, \xi)$.

(2) We will provide a series of conditions under which a sequence $\left(\Psi_{n}\right)_{n}$ of general dissipation potentials with superlinear growth at infinity, not necessarily of form (1.2) (vanishing viscosity) or (1.6) (stochastic approximation), give rise to a viscosity contact potential. Such conditions will amount to requiring that the bipotentials $b_{\Psi_{n}}$ : $[0,+\infty) \times \mathbb{R}^{d} \times \mathbb{R}^{d} \rightarrow[0+\infty]$, associated with the functionals $\Psi_{n}$, and defined by

$$
\mathrm{b}_{\Psi_{n}}(\tau, v, \xi):=\tau \Psi_{n}\left(\frac{v}{\tau}\right)+\tau \Psi_{n}^{*}(\xi) \quad \text { for } \tau>0
$$


(cf. (4.1) ahead), converge in a suitable variational sense to $p$, as $n \rightarrow \infty$. As we will see, such conditions are for instance verified in the case of the vanishing viscosity and of the stochastic approximations.

(3) It will turn out (cf. Theorem 5.2) that under this condition on the bipotentials $b_{\Psi_{n}}$, jointly with a suitable uniform coercivity requirement for the functionals $\left(\Psi_{n}\right)_{n}$, a $\Gamma$-lim inf

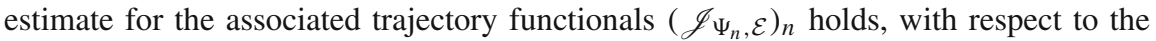
weak topology in $\mathrm{BV}\left([0, T] ; \mathbb{R}^{d}\right)$.

Like previously illustrated for the $\Gamma$-lim inf result in [12], our Theorem 5.2 has the following outcome in terms of evolutionary systems: it implies that limit curves $u \in \mathrm{BV}\left([0, T] ; \mathbb{R}^{d}\right)$ of sequences of solutions $\left(u_{n}\right)_{n}$ to the gradient systems $\left(\Psi_{n}, \mathcal{E}\right)$ (where $\left(\Psi_{n}\right)_{n}$ are dissipation potentials with superlinear growth at infinity that give rise to a viscosity contact potential $p$ in the aforementioned sense) are balanced viscosity solutions to the rate-independent system $\left(\Psi_{0}, \mathrm{p}, \mathcal{E}\right)$, cf. Theorem 5.3 ahead for a precise statement. In other words, we conclude that the gradient systems $\left(\Psi_{n}, \mathcal{E}\right)_{n}$ Evolutionary $\Gamma$-converge to the rate-independent system $\left(\Psi_{0}, p, \mathcal{E}\right)$, using a terminology recently popularized in [34] (cf. also, e.g., [13,40,49-51,54] for limit passages in gradient systems driven by $\Gamma$-converging energy functionals and dissipation potentials).

The proof of the lim sup-estimate in Theorem 5.9 focuses on the specific cases of the vanishing-viscosity and the stochastic approximations. It could be generalized in some directions, cf. Remarks 5.10 and 6.2 ahead. Nonetheless, we have preferred to confine the discussion to the vanishing-viscosity and the stochastic cases, in order to make the calculations more explicit.

Let us, however, mention that in [12] the $\Gamma$-lim sup result was obtained in a much larger generality, for a broader class of dissipation potentials $\Psi_{n}$ that encompass those considered here, with calculations tailored to the one-dimensional context therein. It is not clear to us how to fully extend such calculations in the frame of the approach we have adopted in our multidimensional setup, which, differently from [12], is based on careful computations involving the bipotentials $b_{\Psi_{n}}$ from (1.10). All the more, the proof of the lim sup-estimate in the fully general case, i.e., under the sole condition that the bipotentials $b_{\Psi_{n}}$ from (1.10) variationally converge to $\mathrm{p}$, remains an open problem.

Still, we believe Theorem 5.9 to be relevant. Again, the key observation is that (null)minimizers of the involved functionals of trajectories are solutions of the associated evolutionary problems. Hence, Theorem 5.9 yields a reverse approximation result, Theorem 5.12 ahead, of balanced viscosity solutions of the limiting rate-independent system, by means of solutions of the approximating gradient systems. Such a result $(i)$ extends what was proved in [12] to the multi-dimensional case; (ii) is, to our knowledge, new in the case of the vanishing-viscosity approximation.

Plan of the paper. In Sect. 2, we present the multi-dimensional analogue of the stochastic model considered in [12] and (formally) derive the associated dissipation potential $\Psi_{n}$ and the induced trajectory functional $\mathscr{J}_{\Psi_{n}, \mathcal{E}}$.

Section 3 revolves around an extended notion of balanced viscosity solution to a rateindependent system, based on the concept of viscosity contact potential $p$ introduced in Definition 3.2. This concept of solution is defined in Definition 3.5; its properties are illustrated thereafter.

In Sect. 4, we address the generation of a viscosity contact potential $\mathrm{p}$ starting from a family $\left(\Psi_{n}\right)_{n}$ of dissipation potentials with superlinear growth at infinity, such that the associated bipotentials $\mathrm{b}_{\Psi_{n}}$ from (1.10) variationally converge to $\mathrm{p}$. 


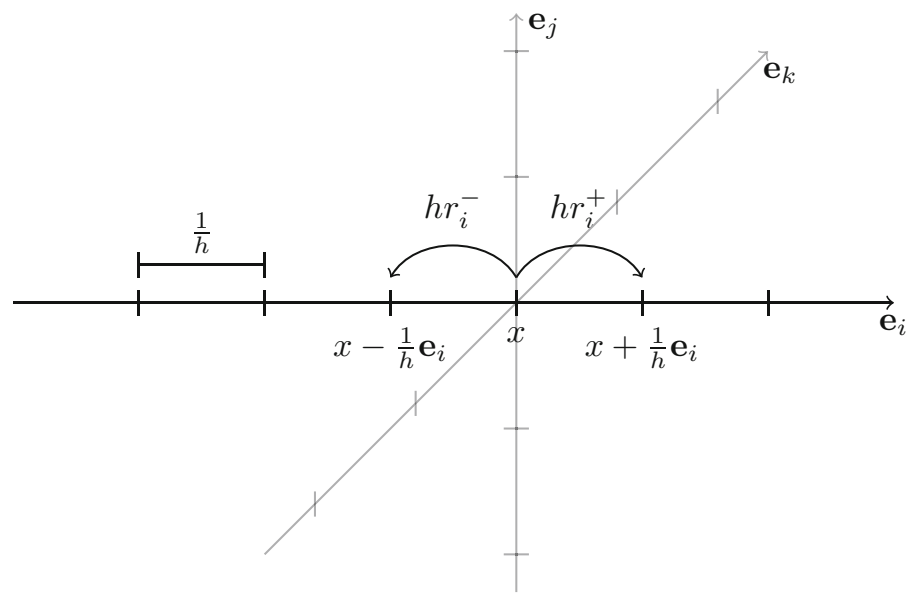
$x \in \frac{1}{h} \mathbb{Z}^{d}$

Fig. 1 A sketch of the jump process on the lattice

Our main results, Theorems 5.2 and 5.9 , along with their consequences Theorems 5.3 and 5.12 , are stated in Sect. 5 and proved throughout Sect. 6.

\section{The stochastic origin of rate-independent systems}

In this section, we briefly describe the multi-dimensional extension of the one-dimensional stochastic model for rate-independent evolution considered in [12].

We consider a jump process $t \mapsto X^{h}(t)$ on a $d$-dimensional lattice, with lattice spacing $\frac{1}{h}$. The evolution of the process can be described as follows: Fix the origin as initial point. If the process is at the position $x$ at time $t$, then it jumps in continuous time to its neighbors $\left(x \pm \frac{1}{m} \mathbf{e}_{i}\right)$ with rate $m r_{i}^{ \pm}$, for $i=1, \ldots, d$, where $\left(\mathbf{e}_{1}, \ldots, \mathbf{e}_{d}\right)$ is the basis of $\mathbb{R}^{d}$, cf. Fig. 1 . The jump rates depend on two parameters $\alpha$ and $\beta$, and on the partial derivatives $\mathrm{D}_{i} \mathcal{E}:=\mathrm{D}_{x_{i}} \mathcal{E}$ of a smooth energy functional $\mathcal{E}:[0, T] \times \mathbb{R}^{d} \rightarrow \mathbb{R}$, namely

$$
r_{i}^{+}(x, t)=\alpha e^{\left.-\beta \mathrm{D}_{i} \mathcal{E}(x, t)\right)}, \quad r_{i}^{-}(x, t)=\alpha e^{\left.\beta \mathrm{D}_{i} \mathcal{E}(x, t)\right)} \quad \text { for } i=1, \ldots, d .
$$

The choice of the stochastic process (and thus of the jump rates $r_{i}^{ \pm}$) reflects Kramers' formula $[10,12,27]$. Given a particle evolving in a wiggly energy landscape with noise, this formula provides an estimate of the rate of jumps from one energy well to the next one.

We are interested in the continuum limit as $h \rightarrow \infty$. With this aim, we apply the method developed by FENG \& KURTZ, cf. [21], to prove large-deviations principles for Markov processes.

As in [12, Sec. 2.5], we will provisionally assume that the jump rates $r^{ \pm}$are constant in space and time and thus derive the expression of the rate function, and then formally substitute (2.1) into it. Following [21], we consider the generator

$$
\Omega_{h} f(x):=\sum_{i=1}^{d}\left[h r_{i}^{+}\left(f\left(x+\frac{1}{h} \mathbf{e}_{i}\right)-f(x)\right)+h r_{i}^{-}\left(f\left(x-\frac{1}{h} \mathbf{e}_{i}\right)-f(x)\right)\right]
$$


of the continuous-time Markov process $X^{h}$ and the nonlinear generator

$$
\begin{aligned}
\left(\mathrm{H}_{h} f\right)(x):= & \frac{1}{h} e^{-h f(x)}\left(\Omega_{h} e^{h f}\right)(x) \\
=\sum_{i=1}^{d}\left[r_{i}^{+}\left(\exp \left(h\left(f\left(x+\frac{1}{h} \mathbf{e}_{i}\right)-f(x)\right)\right)-1\right)\right. & \left.\quad+r_{i}^{-}\left(\exp \left(h\left(f\left(x-\frac{1}{h} \mathbf{e}_{i}\right)-f(x)\right)\right)-1\right)\right] .
\end{aligned}
$$

According to the FENG-KURTZ method, if $\mathrm{H}_{h}$ converges to some $\mathrm{H}$ in a suitable sense, and if the limiting operator $\mathrm{H} f$ depends locally on $\mathrm{D} f$, we can then define the Hamiltonian $H=H(x, \xi)$ through

$$
(\mathrm{H} f)(x)=: H(x, \mathrm{D} f(x)),
$$

and the Lagrangian as the Legendre transform of $H$, namely

$$
L(x, v):=\sup _{\xi \in \mathbb{R}^{d}}(\langle\xi, v\rangle-H(x, \xi)) .
$$

Then, the Markov process satisfies a large-deviations principle, with rate function

$$
\mathscr{J}(u):= \begin{cases}\int_{0}^{T} L\left(u(t), u^{\prime}(t)\right) \mathrm{d} t & \text { if } u \in \mathrm{AC}\left([0, T] ; \mathbb{R}^{d}\right), \\ +\infty & \text { otherwise. }\end{cases}
$$

We may roughly state this principle in the following form:

$$
\operatorname{Prob}\left(\left\{X^{h} \approx x\right\}\right) \approx \exp (-h \mathscr{J}(x)) \quad \text { as } h \rightarrow \infty .
$$

Namely, the probability of finding $X^{h}$ close to some $x$ is close, in a suitable sense, to $\exp (-h \mathscr{J}(x))$ as $h \rightarrow \infty$; in particular, large values of $\mathscr{J}(x)$ imply small probability. The purposefully vague notations $\approx$ and $\approx$ featuring in (2.3) are made precise in [12, Definition 2]. Let us also mention that (2.3) has to be understood as a relation holding in the Skorokhod space $D([0, T])$, namely the space of cadlag functions (right continuous and with limit from left). The FENG-KURTZ method applied in [12] to prove the large-deviations principle for the one-dimensional stochastic model extends to the present multi-dimensional setting.

In our context, it can be seen that

$$
H(x, \xi)=\sum_{i=1}^{d} r_{i}^{+}\left(e^{\xi_{i}}-1\right)+r_{i}^{-}\left(e^{-\xi_{i}}-1\right) .
$$

Then, $L$ is given by

$$
L(x, v)=\sum_{i=1}^{d}\left[v_{i} \log \left(\frac{v_{i}+\sqrt{v_{i}^{2}+4 r_{i}^{+} r_{i}^{-}}}{2 r_{i}^{+}}\right)-\sqrt{v_{i}^{2}+4 r_{i}^{+} r_{i}^{-}}+r_{i}^{+}+r_{i}^{-}\right]
$$

Substituting in (2.4) expression (2.1) for the jump rates, and choosing the parameters

$$
\alpha=\frac{e^{-n A}}{2} \text { and } \beta=n, \quad n \in \mathbb{N},
$$

we obtain

$$
L(x, v)=n\left(\Psi_{n}(v)+\Psi_{n}^{*}(-\mathrm{D} \mathcal{E}(t, x))-\langle-\mathrm{D} \mathcal{E}(t, x), v\rangle\right)
$$


where $\langle\cdot, \cdot\rangle$ denotes the inner product in $\mathbb{R}^{d}$, and $\Psi_{n}: \mathbb{R}^{d} \rightarrow[0,+\infty)$ is given by

$$
\Psi_{n}(v)=\sum_{i=1}^{d} \psi_{n}\left(v_{i}\right)=\sum_{i=1}^{d} \frac{v_{i}}{n} \log \left(\frac{v_{i}+\sqrt{v_{i}^{2}+e^{-2 n A}}}{e^{-n A}}\right)-\frac{1}{n} \sqrt{v_{i}^{2}+e^{-2 n A}}+\frac{e^{-n A}}{n},
$$

and $\Psi_{n}^{*}$ the Legendre transform of $\Psi_{n}$. It can be easily checked that the structure $\Psi_{n}(v)=$ $\sum_{i=1}^{d} \psi_{n}\left(v_{i}\right)$ transfers to the conjugate, hence

$$
\Psi_{n}^{*}(\xi)=\sum_{i=1}^{d} \psi_{n}^{*}\left(\xi_{i}\right)=\sum_{i=1}^{d} \frac{e^{-n A}}{n}\left(\cosh \left(n \xi_{i}\right)-1\right) .
$$

In view of Lemma 3.1 ahead, we can see that, with choice (2.5) for $L$, the (positive) functional $\mathscr{J}$ from (2.2) is minimized by the solutions of the generalized gradient system

$$
\mathrm{D} \Psi_{n}\left(u^{\prime}(t)\right)+\mathrm{D} \mathcal{E}(t, u(t))=0 \quad \text { for a.a. } t \in(0, T) .
$$

Taking into account that $\mathrm{D} \Psi_{n}^{*}=\left(\mathrm{D} \Psi_{n}\right)^{-1}$, the latter rewrites as the ODE system

$$
u_{i}^{\prime}(t)=-\mathrm{D} \psi_{n}^{*}\left(-\mathrm{D}_{i} \mathcal{E}\left(t, u_{i}(t)\right)\right) \quad \text { for a.a. } t \in(0, T), \text { for all } i=1, \ldots, d \text {. }
$$

\section{Viscosity contact potentials and balanced viscosity solutions to rate-independent systems}

The central objective of this section is to introduce and illustrate the main properties of the notion of balanced viscosity solution to a rate-independent system induced by a general viscosity potential. Therefore, Definition 3.5 ahead shall (slightly) extend the definition given in [41], which was based on the concept of vanishing-viscosity contact potential.

\subsection{Preliminaries}

Before introducing the notion of balanced viscosity solution to a rate-independent system and fixing its main properties, we recall some properties of dissipation potentials and provide some basics of the theory of functions of bounded variation; for a more comprehensive tractation, the reader is referred to, e.g., [3,37].

Dissipation potentials. Hereafter, we will call dissipation potential any function

$$
\Psi: \mathbb{R}^{d} \rightarrow[0,+\infty) \text { convex and such that } \Psi(0)=0 .
$$

It follows from the above conditions that the Fenchel-Moreau conjugate $\Psi^{*}$ then fulfills $\Psi^{*}(0)=0 \leq \Psi^{*}(\xi)$ for all $\xi \in \mathbb{R}^{d}$. We will distinguish two cases:

Dissipation potentials with superlinear growth at infinity: Namely, $\Psi$ fulfills

$$
\lim _{\|v\| \rightarrow+\infty} \frac{\Psi(v)}{\|v\|}=+\infty
$$

for some norm $\|\cdot\|$ on $\mathbb{R}^{d}$. For later use, we point out here that, as a consequence of (3.2) one has

$$
\lim _{\tau \downarrow 0} \tau \Psi\left(\frac{v}{\tau}\right)=+\infty \quad \text { for all } v \in \mathbb{R}^{d} \backslash\{0\} .
$$


1-homogeneous dissipation potentials: In what follows, we will denote by $\Psi_{0}$ a dissipation potential

$$
\begin{gathered}
\Psi_{0}: \mathbb{R}^{d} \rightarrow[0,+\infty) \text { convex, 1-positively homogenous }, \\
\text { and non-degenerate, viz. } \Psi_{0}(v)>0 \text { if } v \neq 0 .
\end{gathered}
$$

Thus, for any norm $\|\cdot\|$ on $\mathbb{R}^{d}$

$$
\exists \eta>0 \forall v \in \mathbb{R}^{d}: \eta^{-1}\|v\| \leq \Psi_{0}(v) \leq \eta\|v\| .
$$

Its convex-analysis subdifferential $\partial \Psi_{0}: \mathbb{R}^{d} \rightrightarrows \mathbb{R}^{d}$ at $v \in \mathbb{R}^{d}$ can be characterized by

$$
\zeta \in \partial \Psi_{0}(v) \Leftrightarrow\left\{\begin{array}{l}
\langle\zeta, w\rangle \leq \Psi_{0}(w) \text { for all } w \in \mathbb{R}^{d} \\
\langle\zeta, v\rangle=\Psi_{0}(v) .
\end{array}\right.
$$

Throughout, we will use the notation

$$
K^{*} \text { for the stable set } \partial \Psi_{0}(0) \text {. }
$$

Recall that $\partial \Psi_{0}(v) \subset K^{*}$ for all $v \in \mathbb{R}^{d}$ and that, indeed, $\Psi_{0}$ is the support function of $K^{*}$, namely

$$
\Psi_{0}(v)=\sup _{\zeta \in K^{*}}\langle\zeta, v\rangle, \quad \text { whence } \quad \Psi_{0}^{*}(\xi)=I_{K^{*}}(\xi) .
$$

We conclude with the following Lemma 3.1 that fixes the observation that, in the case of a dissipation potential $\Psi$ with superlinear growth, the solutions of the generalized gradient system $(\Psi, \mathcal{E})$, i.e.,

$$
\partial \Psi\left(u^{\prime}(t)\right)+\mathrm{D} \mathcal{E}(t, u(t)) \ni 0 \quad \text { for a.a. } t \in(0, T),
$$

can be characterized as the (null-)minimizers of the functional of trajectories $\mathcal{J}_{\Psi, \mathcal{E}}$ : $\mathrm{AC}\left([0, T] ; \mathbb{R}^{d}\right) \rightarrow[0,+\infty)$ given by

$$
\begin{aligned}
\mathcal{J}_{\Psi, \mathcal{E}}(u):= & \int_{0}^{T}\left(\Psi(\dot{u}(s))+\Psi^{*}(-\mathrm{D} \mathcal{E}(s, u(s)))\right) \mathrm{d} s+\mathcal{E}(T, u(T))-\mathcal{E}(0, u(0)) \\
& -\int_{0}^{T} \partial_{t} \mathcal{E}(s, u(s)) \mathrm{d} s .
\end{aligned}
$$

Lemma 3.1 Let $\Psi$ have superlinear growth at infinity. Then, a curve $u \in \operatorname{AC}\left([0, T] ; \mathbb{R}^{d}\right)$ is a solution to (3.9) if and only if

$$
0=\mathcal{J}_{\Psi, \mathcal{E}}(u) \leq \mathcal{J}_{\Psi, \mathcal{E}}(v) \quad \text { for all } v \in \mathrm{AC}\left([0, T] ; \mathbb{R}^{d}\right) .
$$

Proof First of all, the positivity of $\mathcal{J}_{\Psi, \mathcal{E}}$ is due to

$$
\begin{aligned}
\int_{0}^{T}\left(\Psi(\dot{v}(s))+\Psi^{*}(-\mathrm{D} \mathcal{E}(s, v(s)))\right) \mathrm{d} s & \geq-\int_{0}^{T}\langle\mathrm{D} \mathcal{E}(s, v(s)), \dot{v}(s)\rangle \mathrm{d} s \\
& =\mathcal{E}(0, v(0))-\mathcal{E}(T, v(T))+\int_{0}^{T} \partial_{t} \mathcal{E}(s, v(s)) \mathrm{d} s,
\end{aligned}
$$

where the first inequality follows from the elementary convex analysis inequality $\Psi(v)+$ $\Psi^{*}(\xi) \geq\langle\xi, v\rangle$, and the last equality by the chain rule. Suppose now that $\mathcal{J}_{\Psi, \mathcal{E}}(u)=0$. Then, again using the chain rule we conclude that

$$
\int_{0}^{T}\left(\Psi(\dot{u}(s))+\Psi^{*}(-\mathrm{D} \mathcal{E}(s, u(s)))-\langle-\mathrm{D} \mathcal{E}(s, u(s)), \dot{u}(s)\rangle\right) \mathrm{d} s=0 .
$$


Since the integrand is positive, we infer that

$$
\Psi(\dot{u}(t))+\Psi^{*}(-\mathrm{D} \mathcal{E}(t, u(t)))-\langle-\mathrm{D} \mathcal{E}(t, u(t)), \dot{u}(t)\rangle=0 \quad \text { for a.a. } t \in(0, T),
$$

which ultimately yields, again by convex analysis, that $u$ solves (3.9). The very same arguments yield that a solution $u$ of (3.9) fulfills $\mathcal{J}_{\Psi, \mathcal{E}}(u)=0$.

BVfunctions Throughout, we will work with functions of bounded variation pointwise defined at every point $t \in[0, T]$. We recall that a function $u$ in $\mathrm{BV}\left([0, T] ; \mathbb{R}^{d}\right)$ admits left and right limits at every $t \in[0, T]$ :

$u\left(t_{-}\right):=\lim _{s \uparrow t} u(s), u\left(t_{+}\right):=\lim _{s \downarrow t} u(s)$, with the convention $u\left(0_{-}\right):=u(0), u\left(T_{+}\right):=u(T)$,

and its pointwise jump set $\mathrm{J}_{u}$ is the at most countable set defined by

$$
\mathrm{J}_{u}:=\left\{t \in[0, T]: u\left(t_{-}\right) \neq u(t) \text { or } u(t) \neq u\left(t_{+}\right)\right\} \supset \operatorname{ess}_{-} \mathrm{J}_{u}:=\left\{t \in[0, T]: u\left(t_{-}\right) \neq u\left(t_{+}\right)\right\} .
$$

We also recall that the distributional derivative $u^{\prime}$ of $u$ is a Radon vector measure that can be decomposed (cf. [3]) into the sum of the three mutually singular measures

$$
u^{\prime}=u_{\mathscr{L}}^{\prime}+u_{\mathrm{C}}^{\prime}+u_{\mathrm{J}}^{\prime}, \quad u_{\mathscr{L}}^{\prime}=\dot{u} \mathscr{L}^{1}, \quad u_{\mathrm{co}}^{\prime}:=u_{\mathscr{L}}^{\prime}+u_{\mathrm{C}}^{\prime} .
$$

Here, $u_{\mathscr{L}}^{\prime}$ is the absolutely continuous part with respect to the Lebesgue measure $\mathscr{L}^{1}$, whose Lebesgue density $\dot{u}$ is the pointwise (and $\mathscr{L}^{1}$-a.e. defined) derivative of $u, u_{\mathrm{J}}^{\prime}$ is a discrete measure concentrated on ess- $\mathrm{J}_{u} \subset \mathrm{J}_{u}$, and $u_{\mathrm{C}}^{\prime}$ is the so-called Cantor part. We will use the notation $u_{\text {co }}^{\prime}:=u_{\mathscr{L}}^{\prime}+u_{\mathrm{C}}^{\prime}$ for the diffuse part of the measure, which does not charge $\mathrm{J}_{u}$.

Given a (non-degenerate) 1-homogeneous dissipation potential $\Psi_{0}$, it induces a notion of (pointwise) total variation for a curve $u \in \mathrm{BV}\left([0, T] ; \mathbb{R}^{d}\right)$ via

$$
\operatorname{Var}_{\Psi_{0}}(u ;[a, b]):=\sup \left\{\sum_{m=1}^{M} \Psi_{0}\left(u\left(t_{m}\right)-u\left(t_{m-1}\right)\right): a=t_{0}<t_{1}<\cdots<t_{M-1}<t_{M}=b\right\}
$$

for any $[a, b] \subset[0, T]$. Therefore, with any $u \in \mathrm{BV}\left([0, T] ; \mathbb{R}^{d}\right)$ we can associate the non-decreasing function $V_{\Psi_{0}}: \mathbb{R} \rightarrow[0,+\infty)$ defined by

$$
V_{\Psi_{0}}(t):= \begin{cases}0 & \text { if } t \leq 0, \\ \operatorname{Var}_{\Psi_{0}}(u ;[0, t]) & \text { if } t \in(0, T), \\ \operatorname{Var}_{\Psi_{0}}(u ;[0, T]) & \text { if } t \geq T .\end{cases}
$$

Its distributional derivative $\mu_{\Psi_{0}}$ is in turn a Radon measure that can be decomposed into a jump part $\mu_{\Psi_{0}, \mathrm{~J}}$, concentrated on $\mathrm{J}_{u}$ and given by

$$
\mu_{\Psi_{0}, \mathrm{~J}}(\{t\})=\Psi_{0}\left(u(t)-u\left(t_{-}\right)\right)+\Psi_{0}\left(u\left(t_{+}\right)-u(t)\right),
$$

and a diffuse part

$$
\mu_{\Psi_{0}, \mathrm{co}}=\mu_{\Psi_{0}, \mathscr{L}}+\mu_{\Psi_{0}, \mathrm{C}} \quad \text { with } \quad \mu_{\Psi_{0}, \mathscr{L}}=\Psi_{0}(\dot{u}) \mathscr{L}^{1}
$$

and $\mathscr{L}^{1}$ the 1 -dimensional Lebesgue measure. There holds

$$
\begin{aligned}
\operatorname{Var}_{\Psi_{0}}(u ;[a, b]) & =\mu \Psi_{0}, \text { co } \\
& =\int_{a}^{b} \Psi_{0}(\dot{u}) \mathrm{d} t+\mu_{\Psi_{0}, \mathrm{C}}([a, b])+\operatorname{Jmp}_{\Psi_{\Psi_{0}}}(u ;[a, b]),
\end{aligned}
$$


with the jump contribution $\operatorname{Jmp}_{\Psi_{0}}(u ;[a, b])$ given by

$$
\begin{aligned}
& \mathrm{Jmp}_{\Psi_{0}}(u ;[a, b]):=\Psi_{0}\left(u\left(a_{+}\right)-u(a)\right)+\mu \Psi_{0}, \mathrm{~J}((a, b))+\Psi_{0}\left(u\left(b_{+}\right)-u(b)\right) \\
& =\Psi_{0}\left(u\left(a_{+}\right)-u(a)\right) \\
& \quad+\sum_{t \in \mathrm{J}_{u} \cap(a, b)}\left(\Psi_{0}\left(u(t)-u\left(t_{-}\right)\right)+\Psi_{0}\left(u\left(t_{+}\right)-u(t)\right)\right)+\Psi_{0}\left(u\left(b_{+}\right)-u(b)\right) .
\end{aligned}
$$

Finally, for later use we recall that a sequence $\left(u_{n}\right)_{n}$ weakly converges in $\mathrm{BV}\left([0, T] ; \mathbb{R}^{d}\right)$ to a curve $u$ (we will write $u_{n} \rightarrow u$ ) if $u_{n}(t) \rightarrow u(t)$ as $n \rightarrow \infty$ for every $t \in[0, T]$ and $\sup _{n} \operatorname{Var}\left(u_{n} ;[0, T]\right) \leq C<\infty$ (in what follows, we shall denote by $\operatorname{Var}(u ;[0, T])$ the total variation of a curve $u$ induced by a generic norm $\|\cdot\|$ on $\left.\mathbb{R}^{d}\right)$, whereas $\left(u_{n}\right)_{n}$ strictly converges in $\mathrm{BV}\left([0, T] ; \mathbb{R}^{d}\right)$ to $u\left(u_{n} \rightarrow u\right)$ if $u_{n} \rightarrow u$ and $\operatorname{Var}\left(u_{n} ;[0, T]\right) \rightarrow \operatorname{Var}(u ;[0, T])$. Finally, a sequence of functionals $\mathscr{G}_{n}: \mathrm{BV}\left([0, T] ; \mathbb{R}^{d}\right) \rightarrow \mathbb{R}$ MosCO-converge to a functional $\mathscr{G}: \mathrm{BV}\left([0, T] ; \mathbb{R}^{d}\right) \rightarrow \mathbb{R}$ with respect to the weak-strict topology in $\mathrm{BV}\left([0, T] ; \mathbb{R}^{d}\right)$ if

(i) $u_{n} \rightarrow u$ weakly in $\mathrm{BV}\left([0, T] ; \mathbb{R}^{d}\right) \Rightarrow \liminf _{n \rightarrow \infty} \mathscr{G}_{n}\left(u_{n}\right) \geq \mathscr{G}(u)$,

(ii) $\forall u \in \mathrm{BV}\left([0, T] ; \mathbb{R}^{d}\right) \exists\left(u_{n}\right)_{n} \subset \mathrm{BV}\left([0, T] ; \mathbb{R}^{d}\right)$ s.t.

$$
\left\{\begin{array}{l}
u_{n} \rightarrow u \text { strictly in } \mathrm{BV}\left([0, T] ; \mathbb{R}^{d}\right), \\
\lim \sup _{n \rightarrow \infty} \mathscr{G}_{n}\left(u_{n}\right) \leq \mathscr{G}(u) .
\end{array}\right.
$$

We refer to [4] for more details on MosCO-convergence.

Viscosity contact potentials. The notion we are going to introduce now lies at the core of the definition of balanced viscosity solution to a rate-independent system, driven by an energy functional $\mathcal{E}$ complying with (E). Indeed, the concept of viscosity contact potential encodes how viscosity enters into the description of the solution behavior at jumps, cf. (3.26) ahead. It is an extension of the notion of vanishing-viscosity contact potential introduced in [41], in that we are augmenting the contact potential defined therein by the time variable. That is why, we have used two different notations, $\mathfrak{p}$ and $p$, respectively, to distinguish the contact potential from [41] from that introduced here. Furthermore, in referring to the contact potential $p$, we will drop the word 'vanishing' in order to highlight that balanced viscosity solutions do not necessarily arise from a vanishing-viscosity approximation, cf. Sect. 5.2.

Definition 3.2 We call a lower semicontinuous function $\mathrm{p}:[0,+\infty) \times \mathbb{R}^{d} \times \mathbb{R}^{d} \rightarrow[0,+\infty]$ a (viscosity) contact potential if it satisfies the following properties:

(1) for every $\tau \geq 0$ there holds $\mathrm{p}(\tau, v, \xi) \geq\langle v$, $\xi\rangle$ for all $(v, \xi) \in \mathbb{R}^{d} \times \mathbb{R}^{d}$;

(2) for every $\xi \in \mathbb{R}^{d}$ the map $(\tau, v) \mapsto \mathrm{p}(\tau, v, \xi)$ is convex and positively 1-homogeneous.

(3) for every $\tau>0$ and $v \in \mathbb{R}^{d}$, the map $\xi \mapsto \mathrm{p}(\tau, v, \xi)$ is convex.

Moreover, we say that $\mathrm{p}$ is non-degenerate if

(4) for every $\tau \geq 0$ there holds $\mathrm{p}(\tau, v, \xi)>0$ if $v \neq 0$.

Finally, given a (non-degenerate) 1-homogeneous dissipation potential $\Psi_{0}$ as in (3.4), we say that $\mathrm{p}$ is $\Psi_{0}$-non-degenerate if

(5) for all $(v, \xi) \in \mathbb{R}^{d} \times \mathbb{R}^{d}$ there holds $\mathrm{p}(0, v, \xi) \geq \Psi_{0}(v)$.

A crucial object related to a (viscosity) contact potential $p$ is the set where the inequality in (1) holds as an equality. We will call it contact set and denote it by

$$
\Lambda_{\mathrm{p}}:=\left\{(\tau, v, \xi) \in[0,+\infty) \times \mathbb{R}^{d} \times \mathbb{R}^{d}: \mathrm{p}(\tau, v, \xi)=\langle v, \xi\rangle\right\},
$$


whereas we will use the notation

$$
\Lambda_{\mathrm{p}, 0}:=\Lambda_{\mathrm{p}} \cap\{0\} \times \mathbb{R}^{d} \times \mathbb{R}^{d}=\left\{(v, \xi) \in \mathbb{R}^{d} \times \mathbb{R}^{d}: \mathrm{p}(0, v, \xi)=\langle v, \xi\rangle\right\} .
$$

Let us point out a first important consequence of the properties defining a contact potential:

Lemma 3.3 For fixed $(\tau, \xi) \in[0,+\infty) \times \mathbb{R}^{d}$, denote by $\partial_{v} p(\tau, \cdot, \xi)(v)$ the (convex analysis) subdifferential at $v$ of the functional $v \mapsto p(\tau, v, \xi)$. Then,

$$
(\tau, v, \xi) \in \Lambda_{p} \Leftrightarrow \xi \in \partial_{v} p(\tau, \cdot, \xi)(v)
$$

Proof Since $v \mapsto \mathrm{p}(\tau, v, \xi)$ is convex and positively homogeneous of degree 1 , we have (cf. (3.6)),

$$
\xi \in \partial_{v} \mathrm{p}(\tau, \cdot, \xi)(v) \text { iff }\left\{\begin{array}{l}
\langle\xi, \tilde{v}\rangle \leq \mathrm{p}(\tau, \tilde{v}, \xi) \text { for all } \tilde{v} \in \mathbb{R}^{d}, \\
\langle\xi, v\rangle=\mathrm{p}(\tau, v, \xi)
\end{array}\right.
$$

and the thesis follows.

Remark 3.4 Observe that, for fixed $\tau \in[0,+\infty)$, the function $\mathrm{p}(\tau, \cdot, \cdot): \mathbb{R}^{d} \times \mathbb{R}^{d} \rightarrow$ $[0,+\infty$ ) enjoys some of the properties of the notion of bipotential (cf., e.g., [6]), which is by definition a functional $\mathrm{b}: \mathbb{R}^{d} \times \mathbb{R}^{d} \rightarrow[0,+\infty]$ convex and lower semicontinuous w.r.t. both variables, separately, and fulfilling $\mathrm{b}(v, \xi) \geq\langle v, \xi\rangle$ for all $(v, \xi) \in \mathbb{R}^{d} \times \mathbb{R}^{d}$, as well as a stronger version of (3.20), namely

$$
(v, \xi) \in \Lambda_{\mathrm{b}} \Leftrightarrow \xi \in \partial_{v} \mathrm{~b}(\cdot, \xi)(v) \Leftrightarrow v \in \partial_{\xi} \mathrm{b}(v, \cdot)(\xi),
$$

where the contact set $\Lambda_{\mathrm{b}}$ is defined similarly as in (3.18).

As discussed in [41], the conditions defining the notion of bipotential, however, seem to be too restrictive for the contact potentials arising in the vanishing-viscosity limit of viscous systems approximating rate-independent evolution. Nonetheless, in Sec. 4 we will see how viscosity contact potentials can in fact be generated, via $\Gamma$-convergence, by bipotentials (in the sense of [6]) associated with families of dissipation potentials.

\subsection{BV solutions to rate-independent systems}

We are now in a position to recall the preliminary definitions at the basis of the concept of balanced viscosity solution; notice that all of them involve the reduced contact potential $\mathrm{p}(0, \cdot, \cdot)$ and the energy functional $\mathcal{E} \in \mathrm{C}^{1}\left([0, T] \times \mathbb{R}^{d}\right)$.

First of all, we introduce the (possibly asymmetric) Finsler distance coming into play in the description of the energetic behavior of a rate-independent system at a jump time: For a fixed $t \in[0, T]$, the Finsler distance induced by $\mathrm{p}$ and $\mathcal{E}$ at the time $t$ is defined for every $u_{0}, u_{1} \in \mathbb{R}^{d}$ by

$$
\begin{aligned}
\Delta_{\mathrm{p}, \mathcal{E}}\left(t ; u_{0}, u_{1}\right):=\inf \left\{\int_{r_{0}}^{r_{1}} \mathrm{p}(0, \dot{\theta}(r),-\mathrm{D} \mathcal{E}(t, \theta(r))) \mathrm{d} r:\right. \\
\left.\quad \theta \in \operatorname{AC}\left(\left[r_{0}, r_{1}\right] ; \mathbb{R}^{d}\right), \theta\left(r_{0}\right)=u_{0}, \theta\left(r_{1}\right)=u_{1}\right\} .
\end{aligned}
$$

Observe that, if $\mathrm{p}$ is a $\Psi_{0}$-non-degenerate contact potential for some 1-positively homogeneous potential $\Psi_{0}$, we clearly have $\Delta_{\mathrm{p}, \mathcal{E}}\left(t ; u_{0}, u_{1}\right) \geq \Delta_{\Psi_{0}}\left(u_{0}, u_{1}\right):=\Psi_{0}\left(u_{1}-u_{0}\right)$. Mimicking notion (3.13) of $\Psi_{0}$-total variation, moving from (3.21) we introduce a notion of total variation that measures the dissipation of a $\mathrm{BV}$-curve at its jump points. Indeed, along the 
footsteps of [41, Def. 3.4] and in analogy with (3.16), for a given curve $u \in \operatorname{BV}\left([0, T] ; \mathbb{R}^{d}\right.$ ) with jump set $\mathrm{J}_{u}$, we define the jump variation of $u$ induced by $(\mathrm{p}, \mathcal{E})$ on an interval $[a, b] \subset[0, T]$ by

$$
\begin{aligned}
\operatorname{Jmp}_{\mathrm{p}, \mathcal{E}}(u ;[a, b]):= & \Delta_{\mathrm{p}, \mathcal{E}}\left(a ; u(a), u\left(a_{+}\right)\right) \\
& +\sum_{t \in \mathrm{J}_{u} \cap(a, b)}\left(\Delta_{\mathrm{p}, \mathcal{E}}\left(t ; u\left(t_{-}\right), u(t)\right)+\Delta_{\mathrm{p}, \mathcal{E}}\left(t ; u(t), u\left(t_{+}\right)\right)\right) \\
& +\Delta_{\mathrm{p}, \mathcal{E}}\left(b ; u\left(b_{-}\right), u(b)\right) .
\end{aligned}
$$

Finally, given a (non-degenerate) 1-positively homogeneous dissipation potential $\Psi_{0}$ and a contact viscosity potential $\mathrm{p}$, the (pseudo-)total variation of a curve $u \in \mathrm{BV}\left([0, T] ; \mathbb{R}^{d}\right.$ ) induced by $\left(\Psi_{0}, \mathrm{p}, \mathcal{E}\right)$ is defined by (cf. (3.15))

$$
\operatorname{Var}_{\Psi_{0}, \mathrm{p}, \mathcal{E}}(u ;[a, b]):=\mu_{\Psi_{0}, \mathrm{co}}([a, b])+\operatorname{Jmp}_{\mathrm{p}, \mathcal{E}}(u ;[a, b]) \text { for any }[a, b] \subset[0, T],
$$

with $\mu_{\Psi_{0} \text {,co }}$ from (3.14) the diffuse part of the total variation measure of the map $t \mapsto$ $\operatorname{Var}_{\Psi_{0}}(u ;[0, t])$. Let us mention that the notation $\operatorname{Var}_{\Psi_{0}, \mathrm{p}, \mathcal{E}}$ is used here with slight abuse, since $\operatorname{Var}_{\Psi_{0}, \mathrm{p}, \mathcal{E}}$ does not enjoy all of the standard properties of total variation functionals, see [41, Rmk. 3.6] for further details. Also observe that, if $\mathrm{p}$ is $\Psi_{0}$-non-degenerate, then we have $\operatorname{Var}_{\Psi_{0}, \mathrm{p}, \mathcal{E}}(u ;[a, b]) \geq \operatorname{Var}_{\Psi_{0}}(u ;[a, b])$.

We are finally in a position to introduce the concept of balanced viscosity solution to the rate-independent system individuated by the triple $\left(\Psi_{0}, \mathrm{p}, \mathcal{E}\right)$. This definition extends $[41$, Def. 4.1].

Definition 3.5 (Balanced viscosity solution) Given a (non-degenerate) 1-homogeneous dissipation potential $\Psi_{0}$ and a (non-degenerate) viscosity contact potential $\mathrm{p}$, we say that a curve $u \in \mathrm{BV}\left([0, T] ; \mathbb{R}^{d}\right)$ is a balanced viscosity $(\mathrm{BV})$ solution to the rate-independent system $\left(\Psi_{0}, \mathrm{p}, \mathcal{E}\right)$ if it fulfills the local stability $\left(\mathrm{S}_{\mathrm{loc}}\right)$ and the $\left(\mathrm{E}_{\Psi_{0}, \mathrm{p}, \mathcal{E}}\right)$-energy-dissipation balance

$$
-\mathrm{DE}(t, u(t)) \in K^{*} \text { for all } t \in[0, T] \backslash \mathrm{J}_{u},
$$

$$
\operatorname{Var}_{\Psi_{0}, \mathrm{p}, \mathcal{E}}(u ;[0, t])+\mathcal{E}(t, u(t))=\mathcal{E}(0, u(0))+\int_{0}^{t} \partial_{t} \mathcal{E}(s, u(s)) \mathrm{d} s \quad \text { for all } t \in[0, T]
$$

with $K^{*}=\partial \Psi_{0}(0)$.

While referring to [41, Sec. 4] and [42, Sec. 3] for a detailed survey of the properties of BV solutions, let us only mention here a few. Firstly, by the analogue of [41, Prop. 4.2], for a BV solution the energy-dissipation balance $\left(\mathrm{E}_{\Psi_{0}, \mathrm{p}, \mathcal{E}}\right)$ indeed holds on every subinterval $[s, t] \subset[0, T]$, i.e.,

$$
\operatorname{Var}_{\Psi_{0}, \mathrm{p}, \mathcal{E}}(u ;[s, t])+\mathcal{E}(t, u(t))=\mathcal{E}(s, u(s))+\int_{s}^{t} \partial_{t} \mathcal{E}(r, u(r)) \mathrm{d} r \quad \text { for all }[s, t] \subset[0, T] .
$$

$$
\left(\mathrm{E}_{\left.\Psi_{0}, \mathrm{p}, \mathcal{E}\right)}\right.
$$

Furthermore, the concept of BV solution yields a thorough description of the energetic behavior of the solution at jumps through the concept of optimal jump transition. For fixed $t \in[0, T]$ and $u_{-}, u_{+} \in \mathbb{R}^{d}$, we call a curve $\theta \in \mathrm{AC}\left([0,1] ; \mathbb{R}^{d}\right.$ ) (up to a rescaling, we may indeed suppose the curves in (3.21) to be defined on $[0,1])$, with $\theta(0)=u_{-}$and 
$\theta(1)=u_{+}$, a $\left(\mathrm{p}, \mathcal{E}_{t}\right)$-optimal transition between $u_{-}$and $u_{+}$if it has constant Finsler velocity $\mathrm{p}(0, \dot{\theta}(\cdot),-\mathrm{D} \mathcal{E}(t, \theta(\cdot)))$ and there holds

$$
\mathcal{E}\left(t, u_{-}\right)-\mathcal{E}\left(t, u_{+}\right)=\Delta_{\mathrm{p}, \mathcal{E}}\left(t ; u_{-}, u_{+}\right)=\mathrm{p}(0, \dot{\theta}(r),-\mathrm{D} \mathcal{E}(t, \theta(r)))>0 \text { for a.a. } r \in(0,1) .
$$

The following result subsumes [41, Prop. 4.6, Thm. 4.7].

Proposition 3.6 Let $u \in \mathrm{BV}\left([0, T] ; \mathbb{R}^{d}\right)$ be a balanced viscosity solution to the rateindependent system $\left(\Psi_{0}, p, \mathcal{E}\right)$. Then, at every jump time $t \in \mathrm{J}_{u}$ there exists a $\left(p, \mathcal{E}_{t}\right)$-optimal transition $\theta^{t}$ between the left and right limits $u_{-}(t)$ and $u_{+}(t)$, such that $\theta^{t}(r)=u(t)$ for some $r \in[0,1]$. Moreover, any optimal jump transition $\theta^{t}$ between $u_{-}(t)$ and $u_{+}(t)$ complies with the contact contact condition

$$
\left(\dot{\theta}^{t}(r),-\mathrm{DE}\left(t, \theta^{t}(r)\right)\right) \in \Lambda_{p, 0} \quad \text { for a.a. } r \in(0,1),
$$

with the contact set $\Lambda_{p, 0}$ from (3.19).

A crucial consequence of (3.25) and of (3.20) from Lemma 3.3 is that any optimal jump transition $\theta^{t}$ complies with the subdifferential inclusion

$$
-\mathrm{D} \mathcal{E}\left(t, \theta^{t}(r)\right) \in \partial_{v} \mathrm{p}\left(0, \cdot,-\mathrm{D} \mathcal{E}\left(t, \theta^{t}(r)\right)\right)\left(\dot{\theta}^{t}(r)\right) \quad \text { for a.a. } r \in(0,1) .
$$

The validity of this flow rule explicitly shows how the contact potential $p$ enters into the description of the solution behavior at jumps.

With the last result of this section, we reformulate the BV solution concept in terms of the null-minimization of a functional defined on BV-trajectories; this will be crucial for the variational convergence analysis developed in Sec. 5. Namely, given a rate-independent system $\left(\Psi_{0}, \mathrm{p}, \mathcal{E}\right)$, we define the trajectory functional $\mathscr{J}_{\Psi_{0}, \mathrm{p}, \mathcal{E}}: \mathrm{BV}\left([0, T] ; \mathbb{R}^{d}\right) \rightarrow(-\infty,+\infty]$ by

$$
\begin{aligned}
& \mathscr{J}_{\Psi_{0}, \mathrm{p}, \mathcal{E}}(u): \\
& =\operatorname{Var}_{\Psi_{0}, \mathrm{p}, \mathcal{E}}(u ;[0, T])+\int_{0}^{T} \Psi_{0}^{*}(-\mathrm{D} \mathcal{E}(t, u(t))) \mathrm{d} t \\
& \quad+\mathcal{E}(T, u(T))-\mathcal{E}(0, u(0))-\int_{0}^{T} \partial_{t} \mathcal{E}(s, u(s)) \mathrm{d} s \\
& =\int_{0}^{T}\left(\Psi_{0}(\dot{u}(s))+\Psi_{0}^{*}(-\mathrm{D} \mathcal{E}(s, u(s)))\right) \mathrm{d} s+\mu_{\Psi_{0}, \mathrm{C}}([0, T])+\operatorname{Jmp}_{\mathrm{p}, \mathcal{E}}(u ;[0, T]) \\
& \quad+\mathcal{E}(T, u(T))-\mathcal{E}(0, u(0))-\int_{0}^{T} \partial_{t} \mathcal{E}(s, u(s)) \mathrm{d} s
\end{aligned}
$$

where the second identity follows from (3.15), with $\dot{u}$ the density of the absolutely continuous part of $u^{\prime}$ w.r.t. the Lebesgue measure $\mathscr{L}^{1}$. We then have the following

Proposition 3.7 A curve $u \in \mathrm{BV}\left([0, T] ; \mathbb{R}^{d}\right)$ is a balanced viscosity solution to the rateindependent system $\left(\Psi_{0}, p, \mathcal{E}\right)$ if and only if

$$
0=\mathscr{J}_{\Psi_{0}, p, \mathcal{E}}(u) \leq \mathscr{J}_{\Psi_{0}, p, \mathcal{E}}(v) \quad \text { for all } v \in \mathrm{BV}\left([0, T] ; \mathbb{R}^{d}\right)
$$

Proof First of all, observe that conditions $\left(\mathrm{S}_{\mathrm{loc}}\right)-\left(\mathrm{E}_{\Psi_{0}, \mathrm{p}, \mathcal{E}}\right)$ are indeed equivalent to $\left(\mathrm{S}_{\mathrm{loc}}^{\prime}\right)-$ $\left(\mathrm{E}_{\Psi_{0}, \mathrm{p}, \mathcal{E}}\right)$, with

$$
-\mathrm{DE}(t, u(t)) \in K^{*} \text { for } \mathscr{L}^{1}-\text { a.a. } t \in(0, T) .
$$


Indeed, if ( $\left.\mathrm{S}_{\mathrm{loc}}^{\prime}\right)$ holds, with a continuity argument one deduces $-\mathrm{DE}(t, u(t)) \in K^{*}$ at all $t \in[0, T] \backslash \mathbf{J}_{u}$.

Clearly, $\left(\mathrm{S}_{\mathrm{loc}}^{\prime}\right)-\left(\mathrm{E}_{\Psi_{0}, \mathrm{p}, \mathcal{E}}\right)$ are then equivalent to

$$
\mathscr{J}_{\Psi_{0}, \mathrm{p}, \mathcal{E}}(u)=0 .
$$

Now, with an argument based on the chain rule for $\mathcal{E}$, one sees (cf. the proof of [42, Cor. 3.4] and of Lemma 3.1) that along a given curve $v \in \mathrm{BV}\left([0, T] ; \mathbb{R}^{d}\right)$ the map $\mathscr{J}_{\Psi_{0}, \mathrm{p}, \mathcal{E}}(v) \geq 0$, so that (3.29) holds if and only if $\mathscr{J}_{\Psi_{0}, \mathrm{p}, \mathcal{E}}(u) \leq 0$, i.e., $u \in \operatorname{Argmin}_{v \in \mathrm{BV}\left([0, T] ; \mathbb{R}^{d}\right)} \mathscr{J}_{\Psi_{0}, \mathrm{p}, \mathcal{E}}(v)$. This concludes the proof.

\section{Generation of viscosity contact potentials via $\Gamma$-convergence}

In this section, we provide a possible procedure to generate a viscosity contact potential via a $\Gamma$-convergence procedure, starting from a family $\left(\Psi_{n}\right)_{n}$ of dissipation potentials with superlinear growth at infinity (cf. (3.2)).

Preliminarily, given a convex dissipation potential $\Psi$, we define the bipotential $b_{\Psi}$ : $[0,+\infty) \times \mathbb{R}^{d} \times \mathbb{R}^{d} \rightarrow[0,+\infty]$ induced by $\Psi$ via

$$
\mathrm{b}_{\Psi}(\tau, v, \xi):=\left\{\begin{array}{ll}
\tau \Psi\left(\frac{v}{\tau}\right)+\tau \Psi^{*}(\xi) & \text { for } \tau>0, \\
0 & \text { for } \tau=0, v=0, \\
+\infty & \text { for } \tau=0 \text { and } v \neq 0,
\end{array}= \begin{cases}\tau \Psi\left(\frac{v}{\tau}\right)+\tau \Psi^{*}(\xi) & \text { for } \tau>0, \\
I_{\{0\}}(v) & \text { for } \tau=0 .\end{cases}\right.
$$

It is immediate to check that

(1) for every $(v, \xi) \in \mathbb{R}^{d} \times \mathbb{R}^{d}$ the map $\tau \mapsto \mathrm{b}_{\Psi}(\tau, v, \xi)$ is convex;

(2) for every $\tau \geq 0$ the functional $(v, \xi) \mapsto \mathrm{b}_{\Psi}(\tau, v, \xi)$ is a bipotential in the sense of [6] (cf. Remark 3.4);

(3) for every $v \neq 0$ and $\xi \in \mathbb{R}^{d}$ with $\Psi^{*}(\xi) \neq 0$, the set $\operatorname{Argmin}_{\tau>0} \mathrm{~b}_{\Psi}(\tau, v, \xi)$ is non-empty,

where the latter property is due to the fact that $\lim _{\tau \downarrow 0} \mathrm{~b}_{\Psi}(\tau, v, \xi)=+\infty$ due to the superlinear growth of $\Psi$, and $\lim _{\tau \uparrow+\infty} \mathrm{b}_{\Psi}(\tau, v, \xi)=+\infty$.

Let us now be given a sequence $\left(\Psi_{n}\right)_{n}$ of dissipation potentials, and let $\left(\mathrm{b}_{\Psi_{n}}\right)_{n}$ be the associated bipotentials. We assume the following.

Hypothesis 4.1 Let $\mathrm{p}:[0,+\infty) \times \mathbb{R}^{d} \times \mathbb{R}^{d} \rightarrow[0,+\infty]$ be defined by

$$
\begin{array}{r}
\mathrm{p}=\Gamma-\liminf _{n} \mathrm{~b}_{\Psi_{n}} \quad \text { i.e., } \quad \mathrm{p}(\tau, v, \xi):=\inf _{\left\{\liminf _{n \rightarrow \infty} \mathrm{b}_{\Psi_{n}}\left(\tau_{n}, v_{n}, \xi_{n}\right):\right.} \\
\left.\tau_{n} \rightarrow \tau, \quad v_{n} \rightarrow v \quad \xi_{n} \rightarrow \xi\right\} .
\end{array}
$$

Then,

$$
\begin{aligned}
& \text { for every } \xi \in \mathbb{R}^{d} \text { there exists }\left(\xi_{n}\right)_{n} \subset \mathbb{R}^{d} \text { with } \xi_{n} \rightarrow \xi \text { and } \\
& \mathrm{p}(\cdot, \cdot, \xi)=\Gamma-\limsup _{n \rightarrow \infty} \mathrm{b}_{\Psi_{n}}\left(\cdot, \cdot, \xi_{n}\right) \quad \text { i.e., } \\
& \mathrm{p}(\tau, v, \xi)=\inf _{\left(\xi_{n}\right)_{n} \subset \mathbb{R}^{d}, \xi_{n} \rightarrow \xi}\left\{\limsup _{n \rightarrow \infty} \mathrm{b}_{\Psi_{n}}\left(\tau_{n}, v_{n}, \xi_{n}\right): \tau_{n} \rightarrow \tau, \quad v_{n} \rightarrow v\right\} .
\end{aligned}
$$

In Sect. 5.2 ahead, we will exhibit two classes of dissipation potentials $\left(\Psi_{n}\right)_{n}$, with superlinear growth at infinity, and associated functionals $p$, complying with Hypothesis 4.1. 
Observe that with (4.3) we are imposing a stronger condition than $\mathrm{p}=\Gamma-\lim \sup _{n \rightarrow \infty} \mathrm{b}_{\Psi_{n}}$, namely we are asking that

$$
\begin{aligned}
\forall \xi \in \mathbb{R}^{d} \exists\left(\xi_{n}\right)_{n} \subset \mathbb{R}^{d}: \xi_{n} \rightarrow \xi \text { and } \\
\forall(\tau, v) \in[0,+\infty) \times \mathbb{R}^{d} \exists\left(\tau_{n}, v_{n}\right)_{n}:\left\{\begin{array}{l}
\tau_{n} \rightarrow \tau, \\
v_{n} \rightarrow v, \\
\lim \sup _{n \rightarrow \infty} \mathrm{b}_{\Psi_{n}}\left(\tau_{n}, v_{n}, \xi_{n}\right) \leq \mathrm{p}(\tau, v, \xi) .
\end{array}\right.
\end{aligned}
$$

This property will play a key role in the proof of Lemma 4.3 below.

The main result of this section, Theorem 4.2, ensures that the functional $p$ generated via (4.2)-(4.3) is a contact potential in the sense of Definition 3.2.

Theorem 4.2 Let $\left(\Psi_{n}\right)_{n}$ be a sequence of dissipation potentials on $\mathbb{R}^{d}$ complying with Hypothesis 4.1. Then, $p$ is a viscosity contact potential according to Definition 3.2, and there exists a 1-homogeneous dissipation potential $\Psi_{0}$ such that

$$
p(\tau, v, \xi) \geq \Psi_{0}(v) \quad \text { for all }(\tau, v, \xi) \in[0,+\infty) \times \mathbb{R}^{d} \times \mathbb{R}^{d} .
$$

Moreover, if the dissipation potentials $\left(\Psi_{n}\right)_{n}$ fulfill the uniform coercivity condition

$$
\begin{aligned}
& \exists M>0,\left(M_{n}\right)_{n} \subset(0,+\infty) \text { s.t. } M_{n} \rightarrow 0 \text { and } \\
& \forall n \in \mathbb{N} \forall v \in \mathbb{R}^{d} \text { there holds } \Psi_{n}(v) \geq M\|v\|-M_{n},
\end{aligned}
$$

with $\|\cdot\|$ any norm in $\mathbb{R}^{d}$, then $\Psi_{0}$ is non-degenerate and $p$ is $\Psi_{0}$-non-degenerate.

We postpone the proof of Theorem 4.2 to the end of this section, after obtaining a series of preliminary lemmas on the structure that the functional $p$ defined by Hypothesis 4.1 inherits from the potentials $\Psi_{n}$.

Lemma 4.3 Assume Hypothesis 4.1. Then, for every $(\tau, v, \xi) \in[0,+\infty) \times \mathbb{R}^{d} \times \mathbb{R}^{d}$ there holds

(1) $p(\tau, v, \xi) \geq\langle v, \xi\rangle$;

(2) the map $(\tau, v) \mapsto p(\tau, v, \xi)$ is convex and positively homogeneous of degree 1 .

Proof Property (1) is an immediate consequence of (4.2), using that for every $n \in \mathbb{N}$ there holds $\mathrm{b}_{\Psi_{n}}(\tau, v, \xi) \geq\langle v, \xi\rangle$ for every $(\tau, v, \xi) \in[0,+\infty) \times \mathbb{R}^{d} \times \mathbb{R}^{d}$.

In order to show that the mapping $\mathrm{p}(\cdot, \cdot, \xi)$ is convex for fixed $\xi$, let $\left(\xi_{n}\right)_{n}$ with $\xi_{n} \rightarrow \xi$ fulfill (4.3). For fixed $\left(\tau_{0}, v_{0}\right)$ and $\left(\tau_{1}, v_{1}\right)$ let $\left(\tau_{n}^{i}, v_{n}^{i}\right)_{n}, i=1,2$, be two associated recovery sequences for $\mathrm{b}_{\Psi_{n}}\left(\cdot, \cdot, \xi_{n}\right)$ as in (4.4). Then, for every $\lambda \in[0,1]$ there holds

$$
\begin{aligned}
& \mathrm{p}\left((1-\lambda) \tau_{0}+\lambda \tau_{1},(1-\lambda) v_{0}+\lambda v_{1}, \xi\right) \\
& \quad \stackrel{(1)}{\leq} \liminf _{n \rightarrow \infty} \mathrm{b}_{\Psi_{n}}\left((1-\lambda) \tau_{n}^{0}+\lambda \tau_{n}^{1},(1-\lambda) v_{n}^{0}+\lambda v_{n}^{1}, \xi_{n}\right) \\
& \quad \stackrel{(2)}{\leq} \limsup _{n \rightarrow \infty}(1-\lambda) \mathrm{b}_{\Psi_{n}}\left(\tau_{n}^{0}, v_{n}^{0}, \xi_{n}\right)+\lambda \mathrm{b}_{\Psi_{n}}\left(\tau_{n}^{1}, v_{n}^{1}, \xi_{n}\right) \\
& \quad \stackrel{(3)}{\leq}(1-\lambda) \mathrm{p}\left(\tau_{0}, v_{0}, \xi\right)+\lambda \mathrm{p}\left(\tau_{1}, v_{1}, \xi\right),
\end{aligned}
$$

where (1) follows from (4.2), (2) from the convexity of the maps $b_{\Psi_{n}}\left(\cdot, \cdot, \xi_{n}\right)$, and (3) from (4.3).

With an analogous argument, one proves that $\mathrm{p}(\cdot, \cdot, \xi)$ is 1-positively homogeneous. 
We now show that, for $\tau>0$ the functional $\mathrm{p}(\tau, \cdot, \cdot)$ has the same form $(4.1)$ as $\mathrm{b}_{\Psi_{n}}(\tau, \cdot, \cdot)$, cf. (4.8).

Lemma 4.4 Assume Hypothesis 4.1. Let $\Psi_{0}: \mathbb{R}^{d} \rightarrow[0+\infty)$ be defined by

$$
\Psi_{0}(v):=p(1, v, 0) .
$$

Then, $\Psi_{0}$ is a 1-positively homogeneous dissipation potential, the sequence $\left(\Psi_{n}\right)_{n} \Gamma$ converges to $\Psi_{0}$, and thus $\left(\Psi_{n}^{*}\right)_{n} \Gamma$-converges to $\Psi_{0}^{*}$. Furthermore,

$$
p(\tau, v, \xi)=\tau \Psi_{0}\left(\frac{v}{\tau}\right)+\tau \Psi_{0}^{*}(\xi) \text { for all } \tau>0 \text { and all }(v, \xi) \in \mathbb{R}^{d} \times \mathbb{R}^{d} .
$$

Proof Observe that $\Psi_{0}$ from (4.7) is convex and 1-homogeneous thanks to item (2) in the statement Lemma 4.3, which obviously yields the convexity of $v \mapsto \mathrm{p}(1, v, 0)$,

It follows from (4.2), applied with the choices $\tau=1$ and $\xi=0$ and with the sequences $\tau_{n} \equiv 1$ and $\xi_{n} \equiv 1$, that $\Psi_{0} \leq \Gamma$ - $\liminf _{n \rightarrow \infty} \Psi_{n}$. Conversely, applying (4.3), we deduce that $\Gamma$ - $\lim \sup _{n \rightarrow \infty} \Psi_{n} \leq \Psi_{0}$. In fact, we use (4.4) with $\tau=1$ and $\xi=0$ to find a sequence $\xi_{n} \rightarrow 0$ and sequences $\tau_{n} \rightarrow 1$ (we may indeed suppose that $\tau_{n} \uparrow 1$ ) and $v_{n} \rightarrow v$ such that

$$
\limsup _{n \rightarrow \infty}\left(\tau_{n} \Psi_{n}\left(\frac{v_{n}}{\tau_{n}}\right)+\tau_{n} \Psi_{n}^{*}\left(\xi_{n}\right)\right) \leq \mathrm{p}(1, v, 0)
$$

In turn, $\lim \sup _{n \rightarrow \infty}\left(\tau_{n} \Psi_{n}\left(\frac{v_{n}}{\tau_{n}}\right)+\tau_{n} \Psi_{n}^{*}\left(\xi_{n}\right)\right) \geq \lim \sup _{n \rightarrow \infty} \tau_{n} \Psi_{n}\left(\frac{v_{n}}{\tau_{n}}\right) \geq \lim \sup _{n \rightarrow \infty}$ $\Psi_{n}\left(v_{n}\right)$, where the latter inequality ensues from the fact that, for any dissipation potential $\Psi$, the map $\tau \mapsto \Psi\left(\frac{v}{\tau}\right)$ is non-increasing for all $v \in \mathbb{R}^{d}$. Combining these two estimates yields $\Gamma$ - $\lim \sup _{n \rightarrow \infty} \Psi_{n} \leq \Psi_{0}$. All in all, we conclude that $\Psi_{0}=\Gamma-\lim _{n \rightarrow \infty} \Psi_{n}$. Then, $\left(\Psi_{n}^{*}\right)_{n} \Gamma$-converges to $\Psi_{0}^{*}$ by $[4$, Thm. 2.18, p. 495]. As a consequence of these convergences and of (4.1), we have (4.8).

Our next two results address the characterization of $\mathrm{p}$ for $\tau=0$, providing a formula for $\mathrm{p}(0, v, w)$ in the two cases $\Psi_{0}^{*}(\xi)<+\infty$ and $\Psi_{0}^{*}(\xi)=+\infty$.

Lemma 4.5 Assume Hypothesis 4.1. If $\Psi_{0}^{*}(\xi)<+\infty$, then

$$
p(0, v, \xi)=\liminf _{\tau \rightarrow 0} \tau \Psi_{0}\left(\frac{v}{\tau}\right)=\Psi_{0}(v) \quad \text { for all } v \in \mathbb{R}^{d} .
$$

Proof It follows from (4.8) and the fact that $\Psi_{0}^{*}(\xi)<+\infty$ that

$$
\mathrm{p}(0, v, \xi) \leq \liminf _{\tau \rightarrow 0} \mathrm{p}(\tau, v, \xi) \leq \liminf _{\tau \rightarrow 0} \tau \Psi_{0}\left(\frac{v}{\tau}\right) .
$$

To prove the converse inequality, we again use that the map $\tau \mapsto \Psi\left(\frac{v}{\tau}\right)$ is non-increasing for every $v \in \mathbb{R}^{d}$. Therefore, for all $0<\tau<\sigma<1$ we have

$$
\tau \Psi\left(\frac{v}{\tau}\right) \geq \sigma \Psi\left(\frac{v}{\sigma}\right) .
$$

Now, let us fix a sequence $\xi_{n} \rightarrow \xi$ for which (4.3) holds, and accordingly a sequence $\left(\tau_{n}, v_{n}\right) \rightarrow(0, v)$ such that $\mathrm{p}(0, v, \xi)=\liminf _{n \rightarrow \infty}\left(\tau_{n} \Psi_{n}\left(v_{n} / \tau_{n}\right)+\tau_{n} \Psi_{n}^{*}\left(\xi_{n}\right)\right)$. It follows from inequality (4.11) applied to the functionals $\Psi_{n}$ that for every $\sigma \in(0,1)$

$$
\liminf _{n \rightarrow \infty}\left(\tau_{n} \Psi_{n}\left(\frac{v_{n}}{\tau_{n}}\right)+\tau_{n} \Psi_{n}^{*}\left(\xi_{n}\right)\right) \geq \liminf _{n \rightarrow \infty}\left(\sigma \Psi_{n}\left(\frac{v_{n}}{\sigma}\right)\right)=\sigma \Psi_{0}\left(\frac{v}{\sigma}\right),
$$


where we have also exploited the positivity of the functionals $\Psi_{n}^{*}$. Therefore, in view of (4.3) we find

$$
\mathrm{p}(0, v, \xi) \geq \sigma \Psi_{0}\left(\frac{v}{\sigma}\right)
$$

and conclude the converse of (4.10) passing to the limit as $\sigma \rightarrow 0$.

Lemma 4.6 Assume Hypothesis 4.1. If $\Psi_{0}^{*}(\xi)=+\infty$, then

$$
p(0, v, \xi)=\Gamma-\liminf _{n \rightarrow \infty} \inf _{\tau>0} b_{\Psi_{n}}(\tau, v, \xi) \quad \text { for all } v \in \mathbb{R}^{d} .
$$

Proof Inequality $\geq$ follows from the definition of $\mathrm{p}$. To prove the converse one, we may suppose that $v \neq 0$, since $\mathrm{p}(0,0, \xi)=0$. Take $\left(v_{n}, \xi_{n}\right) \rightarrow(v, \xi)$ that attains $\Gamma-\liminf _{n \rightarrow \infty} \inf _{\tau>0} \mathrm{~b}_{\Psi_{n}}(\tau, v, \xi)$, i.e., $\inf _{\tau>0} \mathrm{~b}_{\Psi_{n}}\left(\tau, v_{n}, \xi_{n}\right) \rightarrow \Gamma-\liminf _{n \rightarrow \infty} \inf _{\tau>0}$ $\mathrm{b}_{\Psi_{n}}(\tau, v, \xi)$. In particular, $\lim _{n \rightarrow \infty} \Psi_{n}^{*}\left(\xi_{n}\right)=+\infty$. Therefore, we may choose $\bar{\tau}_{n}$ as

$$
\bar{\tau}_{n} \in \operatorname{Argmin}_{\tau>0}\left(\tau \Psi_{n}\left(\frac{v_{n}}{\tau}\right)+\tau \Psi_{n}^{*}\left(\xi_{n}\right)\right) .
$$

Since $\lim \inf _{n \rightarrow \infty} \Psi_{n}^{*}\left(\xi_{n}\right)=+\infty$, it is clear that $\bar{\tau}_{n} \rightarrow 0$, hence

$$
\Gamma-\liminf _{n \rightarrow \infty} \inf _{\tau>0} \mathrm{~b}_{\Psi_{n}}(\tau, v, \xi)=\lim _{n \rightarrow \infty}\left(\bar{\tau}_{n} \Psi_{n}\left(\frac{v_{n}}{\bar{\tau}_{n}}\right)+\bar{\tau}_{n} \Psi_{n}^{*}\left(\xi_{n}\right)\right) \geq \mathrm{p}(0, v, \xi)
$$

thanks to (4.2).

We now prove a pseudo-monotonicity result for $\mathrm{p}$.

Lemma 4.7 Assume Hypothesis 4.1. Then, for every $\tau, \bar{\tau} \in[0,+\infty), v, \bar{v} \in \mathbb{R}^{d}$ and $\xi, \bar{\xi} \in$ $\mathbb{R}^{d}$ we have that

$$
(p(\tau, v, \xi)-p(\tau, v, \bar{\xi}))(p(\bar{\tau}, \bar{v}, \xi)-p(\bar{\tau}, \bar{v}, \bar{\xi})) \geq 0 .
$$

Proof Observe that (4.13) holds for the bipotentials $b_{\Psi_{n}}$ : indeed, in that case, it reduces to $\tau \bar{\tau}\left(\Psi_{n}^{*}(\xi)-\Psi_{n}^{*}(\bar{\xi})\right)^{2} \geq 0$.

Assume that $\mathrm{p}(\tau, v, \xi)>\mathrm{p}(\tau, v, \bar{\xi})$ and choose $\bar{\xi}_{n}$ as in (4.3) with $\left(\tau_{n}, v_{n}\right)$ such that

$$
\left(\tau_{n}, v_{n}, \bar{\xi}_{n}\right) \rightarrow(\tau, v, \bar{\xi}), \quad \mathrm{b}_{\Psi_{n}}\left(\tau_{n}, v_{n}, \bar{\xi}_{n}\right) \rightarrow \mathrm{p}(\tau, v, \bar{\xi})
$$

It follows from Definition (4.2) of $\mathrm{p}$ that $\mathrm{p}(\tau, v, \xi) \leq \liminf _{n \rightarrow \infty} \mathrm{b}_{\Psi_{n}}\left(\tau_{n}, v_{n}, \xi_{n}\right)$ for every sequence $\xi_{n} \rightarrow \xi$ in $\mathbb{R}^{d}$, and for $\left(\tau_{n}, v_{n}\right)$ as in (4.14). Then,

$$
0<\mathrm{p}(\tau, v, \xi)-\mathrm{p}(\tau, v, \bar{\xi}) \leq \liminf _{n \rightarrow \infty}\left(\mathrm{b}_{\Psi_{n}}\left(\tau_{n}, v_{n}, \xi_{n}\right)-\mathrm{b}_{\Psi_{n}}\left(\tau_{n}, v_{n}, \bar{\xi}_{n}\right)\right) .
$$

Therefore, for sufficiently big $n$, we have that

$$
\mathrm{b}_{\Psi_{n}}\left(\tau_{n}, v_{n}, \xi_{n}\right)-\mathrm{b}_{\Psi_{n}}\left(\tau_{n}, v_{n}, \bar{\xi}_{n}\right) \geq 0 .
$$

Now, again in view of (4.3), choose $\xi_{n} \rightarrow \xi$ (notice that (4.15) holds for any sequence $\xi_{n}$ converging to $\xi$ ) and $\bar{\tau}_{n} \rightarrow \bar{\tau}, \bar{v}_{n} \rightarrow \bar{v}$ such that $\lim _{\sup _{n \rightarrow \infty}} \mathrm{b}_{\Psi_{n}}\left(\bar{\tau}_{n}, \bar{v}_{n}, \xi_{n}\right) \leq \mathrm{p}(\bar{\tau}, \bar{v}, \xi)$. Since $\lim \inf _{n \rightarrow \infty} \mathrm{b}_{\Psi_{n}}\left(\bar{\tau}_{n}, \bar{v}_{n}, \bar{\xi}_{n}\right) \geq \mathrm{p}(\bar{\tau}, \bar{v}, \bar{\xi})$ by (4.2), we conclude that

$$
\mathrm{p}(\bar{\tau}, \bar{v}, \xi)-\mathrm{p}(\bar{\tau}, \bar{v}, \bar{\xi}) \geq \limsup _{n \rightarrow \infty}\left(\mathrm{b}_{\Psi_{n}}\left(\bar{\tau}_{n}, \bar{v}_{n}, \xi_{n}\right)-\mathrm{b}_{\Psi_{n}}\left(\bar{\tau}_{n}, \bar{v}_{n}, \bar{\xi}_{n}\right)\right) \geq 0,
$$

taking into account that $\mathrm{b}_{\Psi_{n}}\left(\bar{\tau}_{n}, \bar{v}_{n}, \xi_{n}\right)-\mathrm{b}_{\Psi_{n}}\left(\bar{\tau}_{n}, \bar{v}_{n}, \bar{\xi}_{n}\right) \geq 0$ for sufficiently big $n$ thanks to (4.16) and the previously observed monotonicity property (4.13) for $b_{\Psi_{n}}$. Thus, (4.13) follows. 
Finally, let us consider contact sets associated with the bipotentials $b_{\Psi_{n}}$, i.e.,

$$
\Lambda_{\mathrm{b}_{\Psi_{n}}}:=\left\{(\tau, v, \xi) \in[0,+\infty) \times \mathbb{R}^{d} \times \mathbb{R}^{d}:\langle v, \xi\rangle=\mathrm{b}_{\Psi_{n}}(\tau, v, \xi)\right\} .
$$

Observe that for every $n \in \mathbb{N}$

(1) $\Lambda_{\mathrm{b}_{\Psi_{n}}} \cap\{0\} \times \mathbb{R}^{d} \times \mathbb{R}^{d}=\{0\} \times\{0\} \times \mathbb{R}^{d}$;

(2) for $\tau>0$, if $(\tau, v, \xi) \in \Lambda_{\mathrm{b}_{\Psi_{n}}}$, then $\tau \in \operatorname{Argmin}_{\sigma \in(0,+\infty)}\left(\sigma \Psi_{n}\left(\frac{v}{\sigma}\right)+\sigma \Psi_{n}^{*}(\xi)\right)$.

The following closedness property may be easily derived from (4.2).

Lemma 4.8 Assume Hypothesis 4.1. Then,

$$
\left\{\begin{array}{l}
\left(\tau_{n}, v_{n}, \xi_{n}\right) \in \Lambda_{b_{\Psi_{n}}}, \\
\left(\tau_{n}, v_{n}, \xi_{n}\right) \rightarrow(\tau, v, \xi)
\end{array} \quad \Rightarrow \quad(\tau, v, \xi) \in \Lambda_{p} .\right.
$$

We are now in a position to carry out the proof of Theorem 4.2 by verifying that $p$ complies with properties (1)-(5) from Definition 3.2.

Properties (1) and (2) are guaranteed by Lemma 4.3, whereas (3) ensues from (4.8) in Lemma 4.4. Concerning property (5), observe that (4.5) ensues from (4.8) for $\tau>0$. For $\tau=0$, it directly follows from (4.9) in the case $\Psi_{0}^{*}(\xi)<+\infty$, whereas for $\Psi_{0}^{*}(\xi)=+\infty$ we use the monotonicity property (4.13), giving

$$
(\mathrm{p}(1, v, \xi)-\mathrm{p}(1, v, 0))(\mathrm{p}(0, v, \xi)-\mathrm{p}(0, v, 0)) \geq 0 .
$$

Now, $\mathrm{p}(1, v, \xi)=\Psi_{0}(v)+\Psi_{0}^{*}(\xi)=+\infty$, hence we deduce that $\mathrm{p}(0, v, \xi) \geq \mathrm{p}(0, v, 0) \geq$ $\Psi_{0}(v)$ (here we have used that $\Psi_{0}^{*}(0)=0$ ).

Under additional (4.6), it is immediate to check that $\Psi_{0}$ given by (4.7) is non-degenerate, whence the validity of property (4). This concludes the proof of Theorem 4.2.

\section{Main results}

Let us consider a sequence $\left(\Psi_{n}\right)_{n}$ of dissipation potentials on $\mathbb{R}^{d}$ with superlinear growth at infinity, namely fulfilling (3.2) for every $n \in \mathbb{N}$. A straightforward extension of the by now classical results by COLLI\&VISINTIN for doubly nonlinear evolution equations (cf. $[15,16])$ yields that for every $n \in \mathbb{N}$ there exists at least a solution $u \in \operatorname{AC}\left([0, T] ; \mathbb{R}^{d}\right.$ ) of (the Cauchy problem for) the generalized gradient system $\left(\Psi_{n}, \mathcal{E}\right)$, with $\mathcal{E}$ complying with (E). Namely, $u$ solves the doubly nonlinear differential inclusion

$$
\partial \Psi_{n}(\dot{u}(t))+\mathrm{D} \mathcal{E}(t, u(t)) \ni 0 \quad \text { for a.a. } t \in(0, T) .
$$

As we have seen with Lemma 3.1, the solutions to (5.1) coincide with the null-minimizers for the (positive) functional of trajectories $\mathcal{J}_{\Psi_{n}, \mathcal{E}}: \mathrm{AC}\left([0, T] ; \mathbb{R}^{d}\right) \rightarrow[0,+\infty)$ defined by

$$
\begin{aligned}
\mathcal{J}_{\Psi_{n}, \mathcal{E}}(u):= & \int_{0}^{T}\left(\Psi_{n}(\dot{u}(s))+\Psi_{n}^{*}(-\mathrm{D} \mathcal{E}(s, u(s)))\right) \mathrm{d} s \\
& +\mathcal{E}(T, u(T))-\mathcal{E}(0, u(0))-\int_{0}^{T} \partial_{t} \mathcal{E}(s, u(s)) \mathrm{d} s .
\end{aligned}
$$

The main results of this paper, Theorems 5.2 and 5.9 ahead, concern the Moscoconvergence to the functional $\mathscr{J} \Psi_{0}, \mathrm{p}, \mathcal{E}$ from (3.27), with respect to the weak-strict topology 


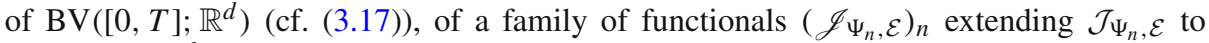
$\mathrm{BV}\left([0, T] ; \mathbb{R}^{d}\right)$. Namely, we define

$$
\begin{aligned}
& \mathscr{J}_{\Psi_{n}, \mathcal{E}}: \operatorname{BV}\left([0, T] ; \mathbb{R}^{d}\right) \rightarrow[0,+\infty] \text { by } \\
& \qquad \mathscr{J}_{\Psi_{n}, \mathcal{E}}(u):= \begin{cases}\mathcal{J}_{\Psi_{n}, \mathcal{E}}(u) & \text { if } u \in \mathrm{AC}\left([0, T] ; \mathbb{R}^{d}\right), \\
+\infty & \text { otherwise. }\end{cases}
\end{aligned}
$$

More precisely, Sect. 5.1 is centered around the $\Gamma$-lim inf result, Theorem 5.2, which implies (cf. Theorem 5.3) the Evolutionary $\Gamma$-convergence of the gradient systems (5.1) to a limiting rate-independent system (understood in the balanced viscosity sense). Theorems 5.2 and 5.3 are valid under the condition that the bipotentials $\left(\mathrm{b}_{\Psi_{n}}\right)_{n}$ associated with the functionals $\left(\Psi_{n}\right)_{n}$ comply with Hypothesis 4.1. In Sect. 5.2, we show that Hypothesis 4.1 is, in particular, verified by two classes of dissipation potentials approximating a 1-homogeneous one, namely for 'vanishing-viscosity' approximations and for the 'stochastic' approximation advanced in [12]. For these two cases (the vanishing-viscosity one further particularized), in Sect. 5.3 we state our $\Gamma$-lim sup result, Theorem 5.9, along with the reverse approximation Theorem 5.12.

With the exception of Proposition 5.8, the proofs of all the upcoming results in this section shall be carried out throughout Sect. 6 .

\subsection{The Г-liminf result}

First of all, let us fix the compactness properties of a sequence $\left(u_{n}\right)_{n} \subset \mathrm{BV}\left([0, T] ; \mathbb{R}^{d}\right)$ with $\sup _{n} \mathscr{J}_{\Psi_{n}, \mathcal{E}}\left(u_{n}\right) \leq C$, assuming that the potentials $\Psi_{n}$ comply with a suitable coercivity property.

Proposition 5.1 Let $\left(\Psi_{n}\right)_{n}$ be a family of dissipation potentials with superlinear growth at infinity and assume that

$$
\exists M_{1}, M_{2}>0 \forall n \in \mathbb{N} \forall v \in \mathbb{R}^{d}: \Psi_{n}(v) \geq M_{1}\|v\|-M_{2}
$$

for some norm $\|\cdot\|$ on $\mathbb{R}^{d}$. Let $\left(u_{n}\right)_{n} \subset \mathrm{BV}\left([0, T] ; \mathbb{R}^{d}\right)$ fulfill $\left\|u_{n}(0)\right\|+\mathscr{J}_{\Psi_{n}, \mathcal{E}}\left(u_{n}\right) \leq C$ for some constant $C>0$, uniform w.r.t. $n \in \mathbb{N}$. Then, there exist a subsequence $k \mapsto n_{k}$ and a curve $u$ such that $u_{n_{k}} \rightarrow u$ in $\mathrm{BV}\left([0, T] ; \mathbb{R}^{d}\right)$.

We are now in a position to state the $\Gamma$ - $\lim$ inf result for the sequence $\left(\mathscr{J}_{\Psi_{n}, \mathcal{E}}\right)_{n}$.

Theorem 5.2 Let $\left(\Psi_{n}\right)_{n}$ be a family of dissipation potentials with superlinear growth at infinity such that the associated bipotentials $\left(b_{\Psi_{n}}\right)_{n}$ comply with Hypothesis 4.1 , with limiting viscosity contact potential p. Let $\Psi_{0}$ be the 1-positively homogeneous dissipation potential defined by $\Psi_{0}(v):=p(1, v, 0)$, and suppose that $\Psi_{0}$ is non-degenerate.

Then, for every $\left(u_{n}\right)_{n}, u \in \mathrm{BV}\left([0, T] ; \mathbb{R}^{d}\right)$, we have that

$$
u_{n} \rightarrow u \text { in } \operatorname{BV}\left([0, T] ; \mathbb{R}^{d}\right) \Rightarrow \liminf _{n \rightarrow \infty} \mathscr{J}_{\Psi_{n}, \mathcal{E}}\left(u_{n}\right) \geq \mathscr{J}_{\Psi_{0}, p, \mathcal{E}}(u)
$$


More precisely, we have as $n \rightarrow \infty$

$$
\begin{aligned}
& \mathcal{E}\left(t, u_{n}(t)\right) \rightarrow \mathcal{E}(t, u(t)) \text { and } \\
& \quad \int_{0}^{t} \partial_{t} \mathcal{E}\left(r, u_{n}(r)\right) \mathrm{d} r \rightarrow \int_{0}^{t} \partial_{t} \mathcal{E}(r, u(r)) \mathrm{d} r \text { for every } t \in[0, T], \\
& \liminf _{n \rightarrow \infty} \int_{s}^{t}\left(\Psi_{n}\left(\dot{u}_{n}(r)\right)+\Psi_{n}^{*}\left(-\mathrm{D} \mathcal{E}\left(r, u_{n}(r)\right)\right)\right) \mathrm{d} r \\
& \quad \geq \operatorname{Var}_{\Psi_{0}, p, \mathcal{E}}(u ;[s, t])+\int_{s}^{t} \Psi_{0}^{*}(-\mathrm{D} \mathcal{E}(t, u(r))) \mathrm{d} r
\end{aligned}
$$

for every $0 \leq s \leq t \leq T$.

A straightforward consequence of Theorem 5.2 is the following result.

Theorem 5.3 Under the assumptions of Theorem 5.2, let $\left(u_{n}\right)_{n} \subset \mathrm{AC}\left([0, T] ; \mathbb{R}^{d}\right)$ fulfill $\mathscr{J}_{\Psi_{n}}\left(u_{n}\right) \leq \varepsilon_{n}$ for every $n \in \mathbb{N}$, for some vanishing sequence $\left(\varepsilon_{n}\right)_{n}$.

Then, any limit point $u$ of $\left(u_{n}\right)_{n}$ with respect to the weak- $\mathrm{BV}\left([0, T] ; \mathbb{R}^{d}\right)$-topology is a balanced viscosity solution to the rate-independent system $\left(\Psi_{0}, p, \mathcal{E}\right)$, and, up to a subsequence, convergences (5.6) and

$$
\begin{aligned}
& \lim _{n \rightarrow \infty} \int_{s}^{t}\left(\Psi_{n}\left(\dot{u}_{n}(r)\right)+\Psi_{n}^{*}\left(-\mathrm{D} \mathcal{E}\left(r, u_{n}(r)\right)\right)\right) \mathrm{d} r \\
& =\operatorname{Var}_{\Psi_{0}, p, \mathcal{E}}(u ;[s, t])+\int_{s}^{t} \Psi_{0}^{*}(-\mathrm{D} \mathcal{E}(t, u(r))) \mathrm{d} r
\end{aligned}
$$

hold for all $0 \leq s \leq t \leq T$.

Remark 5.4 By virtue of Proposition 5.1, under the uniform coercivity condition (5.4) the set of the limit points of the sequence $\left(u_{n}\right)_{n}$ in the statement of Theorem 5.3 is non-empty (if, in addition, $\sup _{n \in \mathbb{N}}\left\|u_{n}(0)\right\| \leq C$ ). If (5.4) is strengthened to (4.6), we also have that the limiting dissipation potential $\Psi_{0}$ is non-degenerate.

\subsection{Examples}

We now focus on two classes of dissipation potentials $\left(\Psi_{n}\right)_{n}$, with superlinear growth at infinity, approximating a 1-positively homogeneous dissipation potential $\Psi_{0}$. In the first case, the dissipation potentials $\Psi_{n}$ are obtained by rescaling from a given dissipation potential $\Psi$ with superlinear growth at infinity and suitably converge to a 1-homogeneous potential $\Psi_{0}$. In the second case, we consider the stochastic model introduced in Sect. 2 and the associated potentials $\Psi_{n}$ given by (2.6): the limiting potential is $\Psi_{0}(v)=A\|v\|_{1}$, where $\|\cdot\|_{1}$ denotes the $L^{1}$-norm on $\mathbb{R}^{d}$

$$
\|v\|_{1}:=\sum_{i=1}^{d}\left|v_{i}\right| .
$$

We will show that in both cases Hypothesis 4.1 is fulfilled. 


\section{The vanishing-viscosity approximation}

We consider the dissipation potentials

$$
\Psi_{n}(v)=\frac{1}{\varepsilon_{n}} \Psi\left(\varepsilon_{n} v\right) \text { for all } v \in \mathbb{R}^{d} \text {, with } \varepsilon_{n} \downarrow 0,
$$

with $\Psi: \mathbb{R}^{d} \rightarrow[0,+\infty)$ a fixed potential with superlinear growth at infinity. We suppose that there exists a 1-homogeneous dissipation potential $\Psi_{0}$ such that

$$
\Psi_{0}(v)=\lim _{n \rightarrow \infty} \Psi_{n}(v)=\lim _{n \rightarrow \infty} \frac{1}{\varepsilon_{n}} \Psi\left(\varepsilon_{n} v\right) \text { for all } v \in \mathbb{R}^{d} .
$$

Example 5.5 In particular, we focus on these two cases (cf. [41, Ex. 2.3]):

(1) $\Psi_{0}$-viscosity: the superlinear dissipation potential $\Psi$ is obtained augmenting $\Psi_{0}$ by a superlinear function of $\Psi_{0}$ itself. Namely, given a convex superlinear function $F_{V}$ : $[0,+\infty) \rightarrow[0,+\infty)$, we set

$$
\begin{aligned}
& \Psi(v):=\Psi_{0}(v)+F_{V}\left(\Psi_{0}(v)\right), \text { whence } \\
& \Psi_{n}(v)=\Psi_{0}(v)+\frac{1}{\varepsilon_{n}} F_{V}\left(\varepsilon_{n} \Psi_{0}(v)\right) \text { for all } v \in \mathbb{R}^{d} .
\end{aligned}
$$

To fix ideas, we may think of $\Psi_{0}(v)=A\|v\|_{1}$ and $F_{V}(\rho)=\frac{1}{2} \rho^{2}$, giving rise to

$$
\Psi_{n}(v)=A\|v\|_{1}+\frac{\varepsilon_{n}}{2} A^{2}\|v\|_{1}^{2} .
$$

(2) 2-norm vanishing viscosity: Let us now consider a norm $\|\cdot\|$ on $\mathbb{R}^{d}$, different from that associated with $\Psi_{0}$. We set

$\Psi(v):=\Psi_{0}(v)+F_{V}(\|v\|), \quad$ whence $\Psi_{n}(v)=\Psi_{0}(v)+\frac{1}{\varepsilon_{n}} F_{V}\left(\varepsilon_{n}\|v\|\right)$ for all $v \in \mathbb{R}^{d}$,

with again $F_{V}:[0,+\infty) \rightarrow[0,+\infty)$ convex and superlinear. In this way, we generate, for example, the dissipation potentials

$$
\Psi_{n}(v)=A\|v\|_{1}+\frac{\varepsilon_{n}}{2}\|v\|_{2}^{2},
$$

with $\|v\|_{2}:=\left(\sum_{i=1}^{d}\left|v_{i}\right|^{2}\right)^{1 / 2}$ and, more in general,

$$
\Psi_{n}(v)=A\|v\|_{1}+\frac{\varepsilon_{n}^{p-1}}{p}\|v\|_{p}^{p} \quad \text { with }\|v\|_{p}:=\left(\sum_{i=1}^{d}\left|v_{i}\right|^{p}\right)^{1 / p} .
$$

This family of dissipation potentials comply with the hypotheses of Theorem 5.2, as shown by the following result.

Proposition 5.6 The dissipation potentials from (5.9) comply with (4.6) and with Hypothesis 4.1, where

$$
\begin{gathered}
p:[0,+\infty) \times \mathbb{R}^{d} \times \mathbb{R}^{d} \rightarrow[0,+\infty] \quad \text { is given by } \\
p(\tau, v, \xi):= \begin{cases}\Psi_{0}(v)+I_{K^{*}}(\xi) & \text { if } \tau>0, \\
\inf _{\varepsilon_{n}>0}\left(\Psi_{n}(v)+\Psi_{n}^{*}(\xi)\right) & \text { if } \tau=0 .\end{cases}
\end{gathered}
$$


The proof can be straightforwardly retrieved from the argument for [41, Lemma 6.1].

Example 5.7 (Example 5.5 continued) Following [41, Rem. 3.1], we explicitly calculate $\mathrm{p}(0, v, \xi)$, using formula (5.15), in the two cases of Example 5.5:

(1) $\Psi_{0}$-viscosity: We have

$$
\mathrm{p}(0, v, \xi):= \begin{cases}\Psi_{0}(v) & \text { if } \xi \in K^{*}, \\ \Psi_{0}(v) \sup _{v \neq 0} \frac{\langle\xi, v\rangle}{\Psi_{0}(v)} & \text { if } \xi \notin K^{*}\end{cases}
$$

Therefore, in the particular case $\Psi_{0}(v)=A\|v\|_{1}$, taking into account that

$$
K^{*}=\bar{B}_{A}^{\infty}(0):=\left\{\xi \in \mathbb{R}^{d}:\|\xi\|_{\infty} \leq A\right\} \quad \text { with }\|v\|_{\infty}:=\max _{i=1, \ldots, d}\left|v_{i}\right|,
$$

we retrieve the formula

$$
\mathrm{p}(0, v, \xi)=\|v\|_{1}\left(A \vee\|\xi\|_{\infty}\right)
$$

(here and in what follows, we use the notation $a \vee b$ for $\max \{a, b\}$ ).

(2) 2-norm vanishing viscosity: In this case, we have

$$
\mathrm{p}(0, v, \xi)=\Psi_{0}(v)+\|v\| \min _{\zeta \in K^{*}}\|\xi-\zeta\|_{*},
$$

where we have used the notation $\|\zeta\|_{*}:=\sup _{v \neq 0} \frac{\langle\zeta, v\rangle}{\|v\|}$.

The stochastic approximation. We now consider the dissipation potentials $\Psi_{n}$ from (2.6), i.e.,

$$
\begin{gathered}
\Psi_{n}(v)=\sum_{i=1}^{d} \psi_{n}\left(v_{i}\right)=\sum_{i=1}^{d} \frac{v_{i}}{n} \log \left(\frac{v_{i}+\sqrt{v_{i}^{2}+e^{-2 n A}}}{e^{-n A}}\right)-\frac{1}{n} \sqrt{v_{i}^{2}+e^{-2 n A}}+\frac{e^{-n A}}{n}, \\
\text { with } \Psi_{n}^{*}(\xi)=\sum_{i=1}^{d} \psi_{n}^{*}\left(\xi_{i}\right)=\sum_{i=1}^{d} \frac{e^{-n A}}{n}\left(\cosh \left(n \xi_{i}\right)-1\right) .
\end{gathered}
$$

Preliminarily, we observe that

$$
\left\{\begin{array}{l}
\Psi_{n}(v) \rightarrow \Psi_{0}(v)=A\|v\|_{1} \text { for all } v \in \mathbb{R}^{d}, \text { and } \Gamma-\lim _{n \rightarrow \infty} \Psi_{n}=\Psi_{0}, \\
\Psi_{n}^{*}(\xi) \rightarrow I_{K^{*}}(\xi) \text { with } K^{*}=\bar{B}_{A}^{\infty}(0) \text { for all } \xi \in \mathbb{R}^{d}, \text { and } \Gamma-\lim _{n \rightarrow \infty} \Psi_{n}^{*}=\Psi_{0}^{*} .
\end{array}\right.
$$

In order to check the above statement, e.g., for $\Psi_{n}(v)$, it is sufficient to recall that $\Psi_{n}(v)=$ $\sum_{i=1}^{d} \psi_{n}\left(v_{i}\right)$, and that the real functions $\left(\psi_{n}\right)_{n}$ pointwise and $\Gamma$-converge to the 1-positively homogeneous potential $\psi_{0}: \mathbb{R} \rightarrow \mathbb{R}$ given by $\psi_{0}(v)=A|v|$. We will now prove that the analogue of Proposition 5.6 holds for the potentials from (5.18).

Proposition 5.8 The dissipation potentials from (5.18) comply with (4.6) and with Hypothesis 4.1 , with limiting viscosity contact potential

$$
p:[0,+\infty) \times \mathbb{R}^{d} \times \mathbb{R}^{d} \rightarrow[0,+\infty] \text { given by } p(\tau, v, \xi):= \begin{cases}\Psi_{0}(v)+I_{K^{*}}(\xi) & \text { if } \tau>0 \\ \|v\|_{1}\left(A \vee\|\xi\|_{\infty}\right) & \text { if } \tau=0\end{cases}
$$


Proof We will split the proof in several claims.

Claim 1: (5.20) holds for $\tau>0$. It follows from the $\Gamma$-convergence properties in (5.19) that $\mathrm{p}=\Gamma-\liminf _{n \rightarrow \infty} \mathrm{b}_{\Psi_{n}}$ fulfills $\mathrm{p}(\tau, v, \xi) \geq \Psi_{0}(v)+I_{K^{*}}(\xi)$ for all $(v, \xi) \in \mathbb{R}^{d} \times \mathbb{R}^{d}$, if $\tau>0$. For the converse inequality, for every $\xi \in \mathbb{R}^{d}$ we take the constant sequence $\xi_{n} \equiv \xi$ and again choose for fixed $(\tau, v) \in(0,+\infty) \times \mathbb{R}^{d}$ the sequences $\tau_{n} \equiv \tau$ and $v_{n} \equiv v$. The pointwise convergences from (5.19) ensure that

$$
\mathrm{p}(\tau, v, \xi) \leq \limsup _{n \rightarrow \infty} \mathrm{b}_{\Psi_{n}}(\tau, v, \xi)=\tau \Psi_{0}\left(\frac{v}{\tau}\right)+\tau I_{K^{*}}(\xi)=\Psi_{0}(v)+I_{K^{*}}(\xi) .
$$

Hence, we conclude that $\mathrm{p}(\tau, v, \xi)=\Psi_{0}(v)+I_{K^{*}}(\xi)$, i.e., the validity of (5.20) for $\tau>0$.

Claim 2: (5.20) holds for $\tau=0$ and $v=0$. In this case, we have to check that $\mathrm{p}(0,0, \xi)=0$, which is equivalent to showing that $p(0,0, \xi) \leq 0$ as the functional $p$ is positive. To this aim, for every fixed $\xi \in \mathbb{R}^{d}$, we observe that for any null sequence $\tau_{n} \downarrow 0$

$$
\mathrm{p}(0,0, \xi) \leq \limsup _{n \rightarrow \infty} \mathrm{b}_{\Psi_{n}}\left(\tau_{n}, 0, \xi\right)=\limsup _{n \rightarrow \infty} \tau_{n} \Psi_{n}^{*}(\xi)
$$

and then we choose $\left(\tau_{n}\right)_{n}$ vanishing fast enough in such a way that the lim sup on the righthand side equals zero.

Claim 3: (5.20) holds for $\tau=0$ and $v \neq 0$. We will split the proof in several (sub-)claims. In the following calculations, taking into account that $\Psi_{n}=\sum_{i=1}^{d} \psi_{n}$ and $\Psi_{n}^{*}=\sum_{i=1}^{d} \psi_{n}^{*}$ with $\psi_{n}: \mathbb{R} \rightarrow \mathbb{R}$ and $\psi_{n}^{*}: \mathbb{R} \rightarrow \mathbb{R}$ even functions, we will often confine the discussion to the case in which $v=\left(v_{1}, \ldots, v_{d}\right)$ fulfills $v_{i} \geq 0$ for all $i=1, \ldots, d$, and analogously for $\xi=\left(\xi_{1}, \ldots, \xi_{d}\right)$.

Moreover, we will need to work with the perturbed bipotentials $b_{\Psi_{n}}^{\delta}:[0,+\infty) \times \mathbb{R}^{d} \times$ $\mathbb{R}^{d} \rightarrow[0,+\infty]$ given by

$$
\mathbf{b}_{\Psi_{n}}^{\delta}(\tau, v, \xi):= \begin{cases}\tau \Psi_{n}\left(\frac{v}{\tau}\right)+\tau \Psi_{n}^{*}(\xi)+\tau \delta & \text { for } \tau>0, \\ 0 & \text { for } \tau=0, v=0, \\ +\infty & \text { for } \tau=0 \text { and } v \neq 0\end{cases}
$$

with $\delta>0$ fixed. We remark that $\operatorname{Argmin}_{\tau>0} \mathrm{~b}_{\Psi_{n}}^{\delta}(\tau, v, \xi) \neq \emptyset$. Indeed, for every fixed $(v, \xi) \in \mathbb{R}^{d} \times \mathbb{R}^{d}$ the functional $\tau \mapsto \mathrm{b}_{\Psi_{n}}^{\delta}(\tau, v, \xi)$ is convex on $(0, \infty)$ and, since $v \neq$ 0 , it fulfills $\lim _{\tau \downarrow 0} \mathrm{~b}_{\Psi_{n}}^{\delta}(\tau, v, \xi)=\lim _{\tau \uparrow \infty} \mathbf{b}_{\Psi_{n}}^{\delta}(\tau, v, \xi)=+\infty$ by the superlinear growth property of the functionals $\Psi_{n}$, cf. also (3.3). A straightforward calculation also shows that for every $\xi \in \mathbb{R}^{d}$ the map $v \mapsto \min _{\tau>0} \mathrm{~b}_{\Psi_{n}}^{\delta}(\tau, v, \xi)$ is 1-positively homogeneous. Therefore, there exists a closed convex set $K_{n, \delta}^{*}(\xi)$ such that

$$
\min _{\tau>0} \mathrm{~b}_{\Psi_{n}}^{\delta}(\tau, v, \xi)=\sup \left\{\langle v, w\rangle: w \in K_{n, \delta}^{*}(\xi)\right\} .
$$

Indeed, it turns out (cf. [41, Thm. A.17]) that

$$
K_{n, \delta}^{*}(\xi)=\left\{w \in \mathbb{R}^{d}: \Psi_{n}^{*}(w) \leq \Psi_{n}^{*}(\xi)+\delta\right\} .
$$

We need an intermediate estimate before proving the $\geq$-inequality in (5.20), i.e., (5.24) below.

Claim 3.1: there holds

$$
\begin{aligned}
& \mathrm{p}(0, v, \xi) \geq \inf \left\{\liminf _{n \rightarrow \infty} \mathrm{b}_{\Psi_{n}}^{\delta}\left(\bar{\tau}_{n}^{\delta}, v_{n}, \xi_{n}\right): v_{n} \rightarrow v, \xi_{n} \rightarrow \xi\right\}, \quad \text { where } \\
& \bar{\tau}_{n}^{\delta} \in \underset{\tau>0}{\operatorname{Argmin}} \mathrm{b}_{\Psi_{n}}^{\delta}\left(\tau, v_{n}, \xi_{n}\right) .
\end{aligned}
$$


This follows from

$$
\begin{aligned}
\mathrm{p}(0, v, \xi) & =\inf \left\{\liminf _{n \rightarrow \infty} \mathrm{b}_{\Psi_{n}}\left(\tau_{n}, v_{n}, \xi_{n}\right): \tau_{n} \rightarrow 0, v_{n} \rightarrow v, \xi_{n} \rightarrow \xi\right\} \\
& =\inf \left\{\liminf _{n \rightarrow \infty}\left(\mathrm{b}_{\Psi_{n}}^{\delta}\left(\tau_{n}, v_{n}, \xi_{n}\right)\right)-\delta \tau_{n}: \tau_{n} \rightarrow 0, v_{n} \rightarrow v, \xi_{n} \rightarrow \xi\right\} \\
& \stackrel{(2)}{\geq} \inf \left\{\liminf _{n \rightarrow \infty} \min _{\tau>0} \mathrm{~b}_{\Psi_{n}}^{\delta}\left(\tau, v_{n}, \xi_{n}\right): v_{n} \rightarrow v, \xi_{n} \rightarrow \xi\right\},
\end{aligned}
$$

where (2) follows from the fact that $\lim _{n \rightarrow \infty} \delta \tau_{n}=0$ for every vanishing sequence $\left(\tau_{n}\right)$.

Claim 3.2: there holds

$$
\mathrm{p}(0, v, \xi) \geq\|v\|_{1}\left(A \vee\|\xi\|_{\infty}\right) .
$$

In view of (5.23), it is sufficient to prove that

$$
\inf \left\{\liminf _{n \rightarrow \infty} \mathrm{b}_{\Psi_{n}}^{\delta}\left(\bar{\tau}_{n}^{\delta}, v_{n}, \xi_{n}\right): v_{n} \rightarrow v, \xi_{n} \rightarrow \xi\right\} \geq\|v\|_{1}\left(A \vee\|\xi\|_{\infty}\right)
$$

Hence, we fix a sequence $\left(v_{n}, \xi_{n}\right) \rightarrow(v, \xi)$ and, for $n$ sufficiently big such that $\frac{1}{n} \log d<A$, define $w_{n} \in \mathbb{R}^{d}$ by

$$
w_{n}:=\left(\left(A \vee\left\|\xi_{n}\right\|_{\infty}\right)-\frac{1}{n} \log d, \cdots,\left(A \vee\left\|\xi_{n}\right\|_{\infty}\right)-\frac{1}{n} \log d\right) .
$$

Taking into account form (2.7) of $\Psi_{n}^{*}$, we estimate

$$
\Psi_{n}^{*}\left(w_{n}\right)=d \frac{e^{-n A}}{n}\left(\cosh \left(n\left\|w_{n}\right\|_{\infty}\right)-1\right)
$$

distinguishing the two cases $\left\|\xi_{n}\right\|_{\infty} \leq A$ and $\left\|\xi_{n}\right\|_{\infty}>A$. In the former situation, it is sufficient to observe that $\left\|w_{n}\right\|_{\infty} \leq A$, so that

$$
\Psi_{n}^{*}\left(w_{n}\right) \leq d \frac{e^{-n A}}{n}(\cosh (n A)-1)=\frac{d}{n}\left(\frac{1+e^{-2 n A}-2 e^{-n A}}{2}\right) \leq \frac{d}{n} \leq \delta
$$

for $n$ sufficiently big. In the case $\left\|\xi_{n}\right\|_{\infty}>A$, we use that

$$
\begin{aligned}
\Psi_{n}^{*}\left(w_{n}\right) & =d \frac{e^{-n A}}{n}\left(\cosh \left(n\left\|\xi_{n}\right\|_{\infty}-\log (d)\right)-1\right) \\
& =d \frac{e^{-n A}}{2 n}\left(e^{n\left\|\xi_{n}\right\|_{\infty}-\log d}+e^{-n\left\|_{n}\right\|_{\infty}+\log d}-2\right) \\
& =\frac{e^{-n A}}{2 n}\left(e^{n\left\|\xi_{n}\right\|_{\infty}}+d^{2} e^{-n\left\|\xi_{n}\right\|_{\infty}}-2 d\right) \\
& =\frac{e^{-n A}}{2 n}\left(e^{n\left\|\xi_{n}\right\|_{\infty}}+e^{-n\left\|\xi_{n}\right\|_{\infty}}-2 d\right)+\frac{e^{-n A}}{2 n}\left(d^{2}-1\right) e^{-n\left\|\xi_{n}\right\|_{\infty}} \leq \Psi_{n}^{*}\left(\xi_{n}\right)+\delta
\end{aligned}
$$

for $n$ sufficiently big such that $\frac{d^{2}-1}{2 n} \leq \delta$. All in all, (5.26) gives that

$$
\Psi_{n}^{*}\left(w_{n}\right) \leq \Psi_{n}^{*}\left(\xi_{n}\right)+\delta,
$$

which implies that $w_{n} \in K_{n, \delta}^{*}\left(\xi_{n}\right)$, for all $n$ sufficiently big. Now, using the representation formula (5.22a) for $\mathrm{b}_{\Psi_{n}}^{\delta}\left(\bar{\tau}_{n}^{\delta}, \cdot, \cdot\right)$, we find

$$
\mathbf{b}_{\Psi_{n}}^{\delta}\left(\bar{\tau}_{n}^{\delta}, v_{n}, \xi_{n}\right) \geq\left\langle v_{n}, w_{n}\right\rangle=\left\|v_{n}\right\|_{1}\left(A \vee\left\|\xi_{n}\right\|_{\infty}\right)-\frac{1}{n} \log d\left\|v_{n}\right\|_{1},
$$


where the last equality follows from the fact that $v_{n}=\left(v_{n}^{1}, \ldots, v_{n}^{d}\right)$ fulfills $v_{n}^{i} \geq 0$ for all $i=1, \ldots, d$. Hence, $\liminf _{n \rightarrow \infty} \mathrm{b}_{\Psi_{n}}^{\delta}\left(\bar{\tau}_{n}^{\delta}, v_{n}, \xi_{n}\right) \geq\|v\|_{1}\left(A \vee\|\xi\|_{\infty}\right)$ and, since the sequences $\left(v_{n}\right)_{n}$ and $\left(\xi_{n}\right)_{n}$ are arbitrary, we conclude (5.25), and thus (5.24).

In order to prove the converse of inequality (5.24), and thus conclude (5.20), we preliminarily need to investigate the properties of the sets $K_{n, \delta}^{*}$.

Claim 3.3: there holds

$\forall \delta>0 \exists n_{\delta} \in \mathbb{N} \forall n \geq n_{\delta} \forall \xi \in \mathbb{R}^{d} \forall w \in K_{n, \delta}^{*}(\xi): \quad\|w\|_{\infty} \leq A \vee\|\xi\|_{\infty}+\frac{1}{n} \log (2 e n \delta)$.

Indeed, every $w \in K_{n, \delta}^{*}(\xi)$ fulfills $\Psi_{n}^{*}(w) \leq \Psi_{n}^{*}(\xi)+\delta$. Using the explicit formula for $\Psi_{n}^{*}$, we obtain that

$$
\frac{e^{-n A}}{n} \cosh \left(n\|w\|_{\infty}\right) \leq \frac{d e^{-n A}}{n} \cosh \left(n\|\xi\|_{\infty}\right)+\delta
$$

whereby

$$
\frac{e^{-n A}}{2 n} e^{n\|w\|_{\infty}} \leq \frac{d e^{-n A}}{2 n} e^{n\|\xi\|_{\infty}}+\frac{d e^{-n A}}{2 n}+\delta \leq \frac{d e^{-n A}}{n} e^{n\|\xi\|_{\infty}}+\delta,
$$

and thus

$$
\|w\|_{\infty} \leq \frac{1}{n} \log \left(2 n \delta e^{n A}+2 d e^{n\|\xi\|_{\infty}}\right) .
$$

Now, doing some algebraic manipulations on the logarithmic term on the right-hand side, we find

$$
\begin{aligned}
\log \left(2 n \delta e^{n A}+2 d e^{n\|\xi\|_{\infty}}\right) & =\log \left(e^{n A+\log n \delta}\left(1+e^{n\left(\|\xi\|_{\infty}-A\right)+\log d-\log n \delta}\right)\right)+\log 2 \\
& \stackrel{(1)}{\leq} \log \left(1+e^{n\left(\|\xi\|_{\infty}-A\right)_{+}}\right)+n A+\log n \delta+\log 2 \\
& \stackrel{(2)}{\leq} n\left(A \vee\|\xi\|_{\infty}\right)+1+\log 2 n \delta,
\end{aligned}
$$

where for (1) we have used that $n \delta>d$ for $n$ sufficiently big and for (2) we have estimated $\log \left(1+e^{n\left(\|\xi\|_{\infty}-A\right)_{+}}\right)=\log \left(e^{n\left(\|\xi\|_{\infty}-A\right)_{+}}\right)+\log \left(e^{-n\left(\|\xi\|_{\infty}-A\right)_{+}}+1\right) \leq \log \left(e^{n\left(\|\xi\|_{\infty}-A\right)_{+}}\right)+1$. Then, (5.27) ensues.

Claim 3.4: for every $(v, \xi) \in \mathbb{R}^{d} \times \mathbb{R}^{d}$ and all sequences $\left(v_{n}\right)_{n}$, $\left(\xi_{n}\right)_{n}$ with $v_{n} \rightarrow v$ and $\xi_{n} \rightarrow \xi$, for every $\bar{\tau}_{n}^{\delta} \in \operatorname{Argmin} \mathrm{b}_{\Psi_{n}}^{\delta}\left(\cdot, v_{n}, \xi_{n}\right)$ there holds

$$
\lim _{n \rightarrow \infty} \bar{\tau}_{n}^{\delta}=0 .
$$

We distinguish two cases: (1) $\Psi_{0}^{*}(\xi)=+\infty$ and (2) $\Psi_{0}^{*}(\xi)=0$.

(1) In the first case, we have $\liminf _{n \rightarrow \infty} \Psi_{n}^{*}\left(\xi_{n}\right)=+\infty$. Then, $\bar{\tau}_{n}^{\delta}$ must be vanishing to 'cancel' the $\tau \Psi_{n}^{*}$-contribution, cf. also the proof of Lemma 4.6.

(2) In the second case, to show (5.28) we will provide an estimate from above for $\bar{\tau}_{n}^{\delta}$ by exploiting the Euler-Lagrange equation for the minimization problem $\min _{\tau>0} b_{\Psi_{n}}^{\delta}$ $(\tau, v, \xi)$. Namely, observe that $\bar{\tau}_{n}^{\delta}$ complies with

$$
0 \in \partial_{\tau} \mathbf{b}_{\Psi_{n}}^{\delta}\left(\cdot, v_{n}, \xi_{n}\right)\left(\bar{\tau}_{n}^{\delta}\right)=\Psi_{n}\left(\frac{v_{n}}{\bar{\tau}_{n}^{\delta}}\right)-\left\langle\mathrm{D} \Psi_{n}\left(\frac{v_{n}}{\bar{\tau}_{n}^{\delta}}\right), \frac{v_{n}}{\bar{\tau}_{n}^{\delta}}\right\rangle+\Psi_{n}^{*}\left(\xi_{n}\right)+\delta .
$$


Using the explicit formula (5.18) for $\Psi_{n}$, we find

$$
\Psi_{n}\left(\frac{v_{n}}{\bar{\tau}_{n}^{\delta}}\right)-\left\langle\mathrm{D} \Psi_{n}\left(\frac{v_{n}}{\bar{\tau}_{n}^{\delta}}\right), \frac{v_{n}}{\bar{\tau}_{n}^{\delta}}\right\rangle=\frac{d e^{-n A}}{n}-\sum_{i} \frac{1}{n} \sqrt{\frac{\left(v_{n}^{i}\right)^{2}}{\left(\bar{\tau}_{n}^{\delta}\right)^{2}}+e^{-2 n A}} .
$$

Therefore, (5.29) yields

$$
\begin{gathered}
n \delta+d e^{-n A}+n \Psi_{n}^{*}\left(\xi_{n}\right)=\sum_{i} \sqrt{\frac{\left(v_{n}^{i}\right)^{2}}{\left(\bar{\tau}_{n}^{\delta}\right)^{2}}+e^{-2 n A}} \leq d \sqrt{\frac{\left\|v_{n}\right\|_{\infty}^{2}}{\left(\bar{\tau}_{n}^{\delta}\right)^{2}}+e^{-2 n A}}, \\
\text { whence }\left(\bar{\tau}_{n}^{\delta}\right)^{2} \leq \frac{d^{2}\left\|v_{n}\right\|_{\infty}^{2}}{n^{2} \delta^{2}+n^{2}\left(\Psi_{n}^{*}\left(\xi_{n}\right)\right)^{2}} \rightarrow 0 \quad \text { for } n \rightarrow \infty
\end{gathered}
$$

We are now in a position to conclude the proof of (5.20).

Claim 3.5: there holds

$$
\mathrm{p}(0, v, \xi) \leq\|v\|_{1}\left(A \vee\|\xi\|_{\infty}\right)
$$

We will in fact prove that

$$
\begin{aligned}
\forall \xi \in \mathbb{R}^{d} \exists\left(\xi_{n}\right)_{n} \subset \mathbb{R}^{d}: \xi_{n} \rightarrow \xi \text { and } \\
\forall v \in \mathbb{R}^{d} \exists\left(\tau_{n}, v_{n}\right)_{n} \text { s.t. }\left\{\begin{array}{l}
\tau_{n} \rightarrow 0, \\
v_{n} \rightarrow v, \\
\limsup _{n \rightarrow \infty} \mathrm{b}_{\Psi_{n}}\left(\tau_{n}, v_{n}, \xi_{n}\right) \leq\|v\|_{1}\left(A \vee\|\xi\|_{\infty}\right) .
\end{array}\right.
\end{aligned}
$$

Taking into account that $\mathrm{p}=\Gamma-\liminf _{n \rightarrow \infty} \mathrm{b}_{\Psi_{n}}$, we will then conclude (5.31). To check (5.32), let us choose the constant recovery sequences $\xi_{n} \equiv \xi$ and $v_{n} \equiv v$, and let $\tau_{n}:=$ $\bar{\tau}_{n}^{\delta} \in \operatorname{Argmin}_{\tau>0} \mathrm{~b}_{\Psi_{n}}^{\delta}(\tau, v, \xi)$. By the previous Claim 3.4, we have that $\tau_{n} \downarrow 0$. Now, in view of the representation formula (5.22a) for $\min _{\tau>0} \mathrm{~b}_{\Psi_{n}}^{\delta}(\tau, v, \xi)$, we can construct a sequence $\left(\tilde{\xi}_{n}\right)_{n} \subset K_{n, \delta}^{*}(\xi)$ such that

$$
\mathrm{b}_{\Psi_{n}}^{\delta}\left(\bar{\tau}_{n}^{\delta}, v, \xi\right) \leq\left\langle v, \tilde{\xi}_{n}\right\rangle+\frac{1}{n} \leq\|v\|_{1}\left(A \vee\|\xi\|_{\infty}\right)+\frac{\|v\|_{1}}{n} \log (2 e n \delta)+\frac{1}{n},
$$

where the second estimate ensues from (5.27). Therefore, $\lim _{\sup _{n \rightarrow \infty}} \mathrm{b}_{\Psi_{n}}^{\delta}\left(\bar{\tau}_{n}^{\delta}, v, \xi\right) \leq$ $\|v\|_{1}\left(A \vee\|\xi\|_{\infty}\right)$. Since $\lim \sup _{n \rightarrow \infty} \mathrm{b}_{\Psi_{n}}^{\delta}\left(\bar{\tau}_{n}^{\delta}, v, \xi\right)=\limsup _{n \rightarrow \infty} \mathrm{b}_{\Psi_{n}}\left(\bar{\tau}_{n}^{\delta}, v, \xi\right)$ as the sequence $\left(\bar{\tau}_{n}^{\delta}\right)_{n}$ is vanishing, we conclude the desired claim (5.32), and thus (5.31).

This finishes the proof of Proposition 5.8.

\subsection{The $\Gamma$-limsup result}

For the $\Gamma$-lim sup counterpart to Theorem 5.2, where we now consider the strict topology in $\mathrm{BV}\left([0, T] ; \mathbb{R}^{d}\right)$, we will focus on the 1-positively homogeneous potential

$$
\Psi_{0}(v)=A\|v\|_{1} \text { with } A>0,
$$

and the two following specific approximations of $\Psi_{0}$ :

vanishing viscosity: the dissipation potentials $\Psi_{n}$ are obtained by augmenting $\Psi_{0}$ by a quadratic term involving a (possibly) different norm $\|\cdot\|$ (cf. 5.12), i.e., 


$$
\Psi_{n}(v)=A\|v\|_{1}+\frac{\varepsilon_{n}}{2}\|v\|^{2} \text { with } \varepsilon_{n} \downarrow 0,
$$

with limiting viscosity contact potential

$$
\mathrm{p}(\tau, v, \xi)= \begin{cases}\Psi_{0}(v)+I_{K^{*}}(\xi) & \text { if } \tau>0, \\ \Psi_{0}(v)+\|v\| \min _{\zeta \in K^{*}}\|\xi-\zeta\|_{*} & \text { if } \tau=0 ;\end{cases}
$$

stochastic approximation: the dissipation potentials $\Psi_{n}$ are given by (5.18), with viscosity contact potential

$$
\mathrm{p}(\tau, v, \xi)= \begin{cases}\Psi_{0}(v)+I_{K^{*}}(\xi) & \text { if } \tau>0 \\ \|v\|_{1}\left(A \vee\|\xi\|_{\infty}\right) & \text { if } \tau=0\end{cases}
$$

Finally, let us mention in advance that, like in [12], for the lim sup-result we will need to impose some enhanced regularity for $\mathcal{E}(t, \cdot)$, namely

$$
\begin{aligned}
& \exists C_{\mathrm{E}}>0 \forall(t, u) \in[0, T] \times \mathbb{R}^{d}:\|\mathrm{D} \mathcal{E}(t, u)\| \leq C_{\mathrm{E}} \text { and } \\
& \quad \mathrm{D} \mathcal{E}(\cdot, u) \text { is uniformly Lipschitz continuous, i.e., } \\
& \exists L_{\mathrm{E}}>0 \forall t_{1}, t_{2} \in[0, T] \forall u \in \mathbb{R}^{d}: \\
& \quad\left\|\mathrm{D} \mathcal{E}\left(t_{1}, u\right)-\mathrm{D} \mathcal{E}\left(t_{2}, u\right)\right\| \leq L_{\mathrm{E}}\left|t_{1}-t_{2}\right| .
\end{aligned}
$$

Theorem 5.9 Let $\mathcal{E}$ comply with (E) and with (5.35), and let the dissipation potentials $\left(\Psi_{n}\right)_{n}$ be given either by (5.33) or by (5.18), with associated limiting bipotential $p$ from (5.33) or (5.34), respectively.

Then, for every $u \in \mathrm{BV}\left([0, T] ; \mathbb{R}^{d}\right)$ there exists a sequence $\left(u_{n}\right)_{n} \subset \mathrm{AC}\left([0, T] ; \mathbb{R}^{d}\right)$, converging to $u$ in the strict topology of $\mathrm{BV}\left([0, T] ; \mathbb{R}^{d}\right)$, such that

$$
\limsup _{n \rightarrow \infty} \mathscr{J}_{\Psi_{n}, \mathcal{E}}\left(u_{n}\right) \leq \mathscr{J}_{\Psi_{0}, p, \mathcal{E}}(u) .
$$

Remark 5.10 In [12], which focused on one-dimensional rate-independent systems, the $\Gamma$ lim sup result was obtained in a much larger generality, for a class of dissipation potentials $\Psi_{n}$ fulfilling suitable growth conditions and other properties. Such properties are satisfied in the two abovementioned particular cases (5.18) and (5.33).

We believe that, to some extent, the results in [12] could be extended to the present multi-dimensional context. Still, we have preferred to confine the discussion to the vanishingviscosity and the stochastic approximations, in order to develop more explicit calculations than those in the proof of [12, Thm. 4.2], significantly exploiting the specific structure of these examples.

Nonetheless, we will briefly comment in Remark 6.2 ahead how the $\Gamma$-lim sup result in the vanishing-viscosity case in fact extends to the broader class of dissipation potentials

$$
\Psi_{n}(v)=A\|v\|_{1}+\frac{\varepsilon_{n}^{p-1}}{p}\|v\|^{p} \text { with } \varepsilon_{n} \downarrow 0, \quad p \in(1,+\infty),
$$

which still have the limiting viscosity contact potential $\mathrm{p}$ from (5.33).

Clearly, Theorems 5.2 and 5.9 yield the Mosco-convergence of the functionals $\left(\mathscr{J}_{\Psi_{n}, \mathcal{E}}\right)_{n}$ to $\mathscr{J} \Psi_{0}, \mathrm{p}, \mathcal{E}$, in the vanishing-viscosity and stochastic cases.

Corollary 5.11 Let $\mathcal{E}$ comply with (E) and with (5.35), and let the dissipation potentials $\left(\Psi_{n}\right)_{n}$ be given either by (5.18), or by (5.33).

Then, the functionals $\left(\mathscr{J}_{\left.\Psi_{n}, \mathcal{E}\right)_{n}}\right.$ Mosco-converge to $\mathscr{J}_{\Psi_{0}, p, \mathcal{E}}$ with respect to the weakstrict topology of $\mathrm{BV}\left([0, T] ; \mathbb{R}^{d}\right)$. 
In the spirit of Theorem 5.3, we also have the following straightforward consequence of Theorem 5.9, of Lemma 3.1, and of Proposition 3.7. Theorem 5.12, whose proof is omitted, is a reverse approximation result.

Theorem 5.12 Let $\mathcal{E}$ comply with (E) and with (5.35). Consider the 1-homogeneous potential $\Psi_{0}(v)=A\|v\|_{1}$.

Consider the viscosity contact potential $p$ from (5.33). Then, for every balanced viscosity solution $u \in \mathrm{BV}\left([0, T] ; \mathbb{R}^{d}\right)$ to the rate-independent system $\left(\Psi_{0}, p, \mathcal{E}\right)$, there exists a sequence $\left(u_{n}\right)_{n} \subset \mathrm{AC}\left([0, T] ; \mathbb{R}^{d}\right)$ of solutions to the gradient systems $\left(\Psi_{n}, \mathcal{E}\right)$, with the dissipation potentials $\left(\Psi_{n}\right)_{n}$ given by $\Psi_{n}(v)=A\|v\|_{1}+\frac{\varepsilon_{n}}{2}\|v\|^{2}$ for all $n \in \mathbb{N}$, where $\left(\varepsilon_{n}\right)_{n} \subset(0,+\infty)$ is any vanishing sequence as $n \rightarrow \infty$, such that $u_{n} \rightarrow u$ as $n \rightarrow \infty$ strictly in $\mathrm{BV}\left([0, T] ; \mathbb{R}^{d}\right)$.

A completely analogous statement holds with the viscosity contact potential p from (5.34), and the dissipation potentials $\left(\Psi_{n}\right)_{n}$ from (5.18).

\section{Proofs}

In what follows, we will denote by $C$ a generic positive constant independent of $n$, whose meaning may vary even within the same line.

We will just outline the argument for the proof of Proposition 5.1, referring to the argument for [41, Thm. 4.1] (see also [12, Thm. 4.2]) for all details. Combining the information that $\mathscr{J}_{\Psi_{n}, \mathcal{E}}\left(u_{n}\right) \leq C$ with the power control condition from (E), we find that

$$
\int_{0}^{T}\left(\Psi_{n}(\dot{u}(s))+\Psi_{n}^{*}(-\mathrm{D} \mathcal{E}(s, u(s)))\right) \mathrm{d} s+\mathcal{E}\left(T, u_{n}(T)\right) \leq C+\int_{0}^{T} C_{1}\left|\mathcal{E}\left(s, u_{n}(s)\right)\right| \mathrm{d} s,
$$

where we have also used that $\left\|u_{n}(0)\right\| \leq C$, and thus $\sup _{n}\left|\mathcal{E}\left(0, u_{n}(0)\right)\right| \leq C$. Taking into account that both $\Psi_{n}$ and $\Psi_{n}^{*}$ are positive, via the Gronwall Lemma we deduce from the above inequality that $\sup _{t \in[0, T]}\left|\mathcal{E}\left(t, u_{n}(t)\right)\right| \leq C$, whence $\sup _{t \in[0, T]}\left|\partial_{t} \mathcal{E}\left(t, u_{n}(t)\right)\right| \leq C$. Hence,

$$
\int_{0}^{T}\left(\Psi_{n}\left(\dot{u}_{n}(s)\right)+\Psi_{n}^{*}(-\mathrm{DE}(s, u(s)))\right) \mathrm{d} s \leq C,
$$

which implies, thanks to the coercivity (5.4), that $\operatorname{Var}\left(u_{n} ;[0, T]\right) \leq C$. Then, the thesis readily follows from the Helly theorem.

Before developing the proof of Theorem 5.2, we preliminarily give the following lower semicontinuity result, in the spirit of [41, Lemma 4.3]).

Lemma 6.1 Let $m, d \geq 1$ and $\mathfrak{F}_{n}, \mathfrak{F}_{\infty}: \mathbb{R}^{m} \times \mathbb{R}^{d} \rightarrow[0,+\infty)$ be normal integrands (i.e., for $n \in \mathbb{N} \cup\{\infty\}$ the functionals $\mathfrak{F}_{n}$ are measurable, and for every $v \in \mathbb{R}^{m}$ the mappings $\xi \mapsto \mathfrak{F}_{n}(v, \xi)$ are lower semicontinuous) such that

(1) for fixed $\xi \in \mathbb{R}^{d}$ the functionals $\mathfrak{F}_{n}(\cdot, \xi)$ are convex for every $n \in \mathbb{N} \cup\{\infty\}$,

(2) there holds

$$
\Gamma-\liminf _{n \rightarrow \infty} \mathfrak{F}_{n} \geq \mathfrak{F}_{\infty} \quad \text { in } \mathbb{R}^{m} \times \mathbb{R}^{d} .
$$

Let $I$ be a bounded interval in $\mathbb{R}$, and let $w_{n}, w: I \rightarrow \mathbb{R}^{m}$ fulfill $w_{n} \rightarrow w$ in $L^{1}\left(I ; \mathbb{R}^{m}\right)$, and $\xi_{n}, \xi: I \rightarrow \mathbb{R}^{d}$ fulfill $\xi_{n}(s) \rightarrow \xi(s)$ for almost all $s \in I$. Then,

$$
\liminf _{n \rightarrow \infty} \int_{I} \mathfrak{F}_{n}\left(w_{n}(s), \xi_{n}(s)\right) \mathrm{d} s \geq \int_{I} \mathfrak{F}_{\infty}(w(s), \xi(s)) \mathrm{d} s .
$$


Proof We introduce the functional

$$
\overline{\mathfrak{F}}: \mathbb{N} \cup\{\infty\} \times \mathbb{R}^{m} \times \mathbb{R}^{d}, \quad \overline{\mathfrak{F}}(n, w, \xi):= \begin{cases}\mathfrak{F}_{n}(w, \xi) & \text { for } n \in \mathbb{N}, \\ \mathfrak{F}_{\infty}(w, \xi) & \text { for } n=\infty .\end{cases}
$$

It follows from (6.1) that $\overline{\mathfrak{F}}$ is lower semicontinuous on $\mathbb{N} \cup\{\infty\} \times \mathbb{R}^{m} \times \mathbb{R}^{d}$; hence, it is a positive normal integrand. Then, (6.2) follows from the Ioffe Theorem, cf. [24] and also, e.g., [53, Thm. 21].

Proof of Theorem 5.2. Let $\left(u_{n}\right)_{n} \subset \mathrm{BV}\left([0, T] ; \mathbb{R}^{d}\right)$ be a sequence weakly converging to $u \in \mathrm{BV}\left([0, T], \mathbb{R}^{d}\right)$. We may suppose that $\liminf _{n \rightarrow \infty} \mathscr{J}_{\Psi_{n}, \mathcal{E}}\left(u_{n}\right)<+\infty$, as otherwise there is nothing to prove. Therefore, up to a subsequence we have $\mathscr{J}_{\Psi_{n}, \mathcal{E}}\left(u_{n}\right) \leq C$, in particular yielding that $u_{n} \in \mathrm{AC}\left([0, T] ; \mathbb{R}^{d}\right)$ for every $n \in \mathbb{N}$. With the very same arguments as in the proof of Prop. 5.1, also based on the power control (E), we see that each contribution to $\mathscr{J}_{\Psi_{n}, \mathcal{E}}\left(u_{n}\right)$ is itself bounded. Convergences (5.6) follow from the pointwise convergence of $\left(u_{n}\right)_{n}$, the fact that $\mathcal{E} \in \mathrm{C}^{1}\left([0, T] \times \mathbb{R}^{d}\right)$, and the Lebesgue dominated convergence theorem, recalling that $\left(u_{n}\right)_{n}$ is bounded in $L^{\infty}\left(0, T ; \mathbb{R}^{d}\right)$. Moreover, we have that $\mathrm{DE}\left(t, u_{n}(t)\right) \rightarrow \mathrm{DE}(t, u(t))$ for every $t \in[0, T]$. Then, taking into account that the functionals $\left(\Psi_{n}^{*}\right)_{n} \Gamma$-converge to $\Psi_{0}^{*}$, we can apply Lemma 6.1 to the functionals $\mathfrak{F}_{n}(w, \xi):=\Psi_{n}^{*}(\xi)$ and $\mathfrak{F}(w, \xi):=\Psi_{0}^{*}(\xi)$ to obtain

$$
\begin{gathered}
\liminf _{n \rightarrow \infty} \int_{0}^{T} \Psi_{n}^{*}\left(-\mathrm{D} \mathcal{E}\left(t, u_{n}(t)\right)\right) \mathrm{d} t \geq \int_{0}^{T} \Psi_{0}^{*}(-\mathrm{D} \mathcal{E}(t, u(t))) \mathrm{d} t, \\
\text { whence }-\mathrm{D} \mathcal{E}(t, u(t)) \in K^{*} \text { for a.a. } t \in(0, T) .
\end{gathered}
$$

Define the nonnegative finite measures on $[0, T]$

$$
v_{n}:=\Psi_{n}\left(\dot{u}_{n}(\cdot)\right) \mathscr{L}^{1}+\Psi_{n}^{*}\left(-\mathrm{D} \mathcal{E}\left(\cdot, u_{n}(\cdot)\right)\right) \mathscr{L}^{1} \doteq \mu_{n}+\eta_{n} .
$$

Up to extracting a subsequence, we can suppose that they weakly* converge in duality with $\mathrm{C}^{0}([0, T])$ to a positive measure

$$
v=\mu+\eta \quad \text { with } \eta \geq \Psi_{0}^{*}(-\mathrm{DE}(\cdot, u(\cdot))) \mathscr{L}^{1} .
$$

Let us now preliminarily show that

$$
v \geq \Psi_{0}(\dot{u}) \mathscr{L}^{1}+\mu \Psi_{0}, \mathrm{C} .
$$

For this, we shall in fact observe that $\mu \geq \Psi_{0}(\dot{u}) \mathscr{L}^{1}+\mu_{\Psi_{0}, \mathrm{C}}$. This will follow upon proving that

$$
\mu([\alpha, \beta])=\lim _{n \rightarrow \infty} \int_{\alpha}^{\beta} \Psi_{n}\left(\dot{u}_{n}(t)\right) \mathrm{d} t \geq \operatorname{Var}_{\Psi_{0}}(u ;[\alpha, \beta]) \quad \text { for every }[\alpha, \beta] \subset[0, T] .
$$

Indeed, let us fix a partition $t_{0}=\alpha<t_{1}<\ldots<t_{k}=\beta$ of $[\alpha, \beta]$ and notice that

$$
\begin{aligned}
\lim _{n \rightarrow \infty} \int_{\alpha}^{\beta} \Psi_{n}\left(\dot{u}_{n}(t)\right) \mathrm{d} t & =\lim _{n \rightarrow \infty} \sum_{m=1}^{k} \int_{t_{m-1}}^{t_{m}} \Psi_{n}\left(\dot{u}_{n}(t)\right) \mathrm{d} t \\
& \stackrel{(1)}{\geq} \liminf _{n \rightarrow \infty} \sum_{m=1}^{k}\left(t_{m}-t_{m-1}\right) \Psi_{n}\left(\frac{\int_{t_{m-1}}^{t_{m}} \dot{u}_{n}(t) \mathrm{d} t}{t_{m}-t_{m-1}}\right) \\
& =\liminf _{n \rightarrow \infty} \sum_{m=1}^{k}\left(t_{m}-t_{m-1}\right) \Psi_{n}\left(\frac{u_{n}\left(t_{m}\right)-u_{n}\left(t_{m-1}\right)}{t_{m}-t_{m-1}}\right)
\end{aligned}
$$




$$
\begin{aligned}
& \stackrel{(2)}{\geq} \sum_{m=1}^{k}\left(t_{m}-t_{m-1}\right) \Psi_{0}\left(\frac{u\left(t_{m}\right)-u\left(t_{m-1}\right)}{t_{m}-t_{m-1}}\right) \\
& \stackrel{(3)}{=} \sum_{m=1}^{k} \Psi_{0}\left(u\left(t_{m}\right)-u\left(t_{m-1}\right)\right),
\end{aligned}
$$

where (1) follows from the Jensen inequality, (2) from the fact that the potentials $\left(\Psi_{n}\right)_{n}$ $\Gamma$-converge to $\Psi_{0}$ (cf. Lemma 4.4), and (3) from the 1-positive homogeneity of $\Psi_{0}$. Since the partition of $[\alpha, \beta]$ is arbitrary, we conclude (6.5).

However, we need to improve (6.4) by obtaining a finer characterization for the jump part of $v$. We will in fact prove that

$$
v(\{t\}) \geq \Delta_{\mathrm{p}, \mathcal{E}}\left(t ; u\left(t_{-}\right), u\left(t_{+}\right)\right) \quad \text { for every } t \in \mathrm{J}_{u}
$$

by adapting the argument in the proof of [42, Prop. 7.3]. To this end, for fixed $t \in \mathrm{J}_{u}$ let us pick two sequences $h_{n}^{-} \uparrow t$ and $h_{n}^{+} \downarrow t$ such that $u_{n}\left(h_{n}^{-}\right) \rightarrow u\left(t_{-}\right)$and $u_{n}\left(h_{n}^{+}\right) \rightarrow u\left(t_{+}\right)$. Define $\mathrm{s}_{n}:\left[h_{n}^{-}, h_{n}^{+}\right] \rightarrow \mathbb{R}$ by

$$
\mathrm{s}_{n}(h):=c_{n}\left(h-h_{n}^{-}+\int_{h_{n}^{-}}^{h}\left(\Psi_{n}\left(\dot{u}_{n}(t)\right)+\Psi_{n}^{*}\left(-\mathrm{D} \mathcal{E}\left(t, u_{n}(t)\right)\right)\right) \mathrm{d} t\right), \quad h \in\left[h_{n}^{-}, h_{n}^{+}\right],
$$

where the normalization constant $c_{n}$ is chosen in such a way that $\mathrm{s}_{n}\left(h_{n}^{+}\right)=1$. Therefore, $\mathrm{s}_{n}$ takes values in $[0,1]$. Observe that for every $n$ the function $s_{n}$ is strictly increasing and thus invertible, and let

There holds

$$
\mathrm{t}_{n}:=\mathrm{s}_{n}^{-1}:[0,1] \rightarrow\left[h_{n}^{-}, h_{n}^{+}\right] \text {and } \vartheta_{n}:=u_{n} \circ \mathrm{t}_{n} .
$$

$$
\begin{aligned}
\dot{\mathrm{t}}_{n}(s)+\left\|\dot{\vartheta}_{n}\right\|_{1}(s) & =\frac{1+\left\|\dot{u}_{n}\right\|_{1}\left(\mathrm{t}_{n}(s)\right)}{c_{n}\left(1+\Psi_{n}\left(\dot{u}_{n}\left(\mathrm{t}_{n}(s)\right)\right)+\Psi_{n}^{*}\left(-\mathrm{D} \mathcal{E}\left(\mathrm{t}_{n}(s), u_{n}\left(\mathrm{t}_{n}(s)\right)\right)\right)\right.} \\
& \leq C \quad \text { for a.a. } s \in(0,1) .
\end{aligned}
$$

Now, by the upper semicontinuity property of the weak*-convergence of measures on closed sets, we have

$$
\begin{aligned}
v(\{t\}) & \geq \limsup _{n \rightarrow \infty} v_{n}\left(\left[h_{n}^{-}, h_{n}^{+}\right]\right) \geq \liminf _{n \rightarrow \infty} \int_{h_{n}^{-}}^{h_{n}^{+}}\left(\Psi_{n}\left(\dot{u}_{n}(t)\right)+\Psi_{n}^{*}\left(-\mathrm{D} \mathcal{E}\left(t, u_{n}(t)\right)\right)\right) \mathrm{d} t \\
& \stackrel{(1)}{=} \liminf _{n \rightarrow \infty} \int_{0}^{1}\left(\Psi_{n}\left(\dot{u}_{n}\left(\mathrm{t}_{n}(s)\right)\right)+\Psi_{n}^{*}\left(-\mathrm{D} \mathcal{E}\left(\mathrm{t}_{n}(s), u_{n}\left(\mathrm{t}_{n}(s)\right)\right)\right)\right) \dot{\mathrm{t}}_{n}(s) \mathrm{d} s \\
& \stackrel{(2)}{=} \liminf _{n \rightarrow \infty} \int_{0}^{1} \mathrm{~b}_{\Psi_{n}}\left(\dot{\mathrm{t}}_{n}(s), \dot{\vartheta}_{n}(s),-\mathrm{D} \mathcal{E}\left(\mathrm{t}_{n}(s), \vartheta_{n}(s)\right)\right) \mathrm{d} s
\end{aligned}
$$

where (1) follows from a change of variables, and (2) from the very Definition (4.1) of $\mathrm{b}_{\Psi_{n}}$. Now, it follows from (6.8) and from the fact that the range of $\mathrm{t}_{n}$ is $\left[h_{n}^{-}, h_{n}^{+}\right]$that there exists $(\mathrm{t}, \vartheta) \in \mathrm{C}_{\text {lip }}^{0}\left([0,1] ;[0, T] \times \mathbb{R}^{d}\right)$ such that, up to a not relabeled subsequence,

$$
\begin{aligned}
& \mathrm{t}_{n}(s) \rightarrow \mathrm{t}(s) \equiv t, \quad \vartheta_{n}(s) \rightarrow \vartheta(s) \text { for all } s \in[0,1], \\
& \dot{\mathrm{t}}_{n} \rightarrow^{*} 0 \text { in } L^{\infty}(0,1), \quad \dot{\vartheta}_{n} \rightarrow^{*} \dot{\vartheta} \text { in } L^{\infty}\left(0,1 ; \mathbb{R}^{d}\right), \\
& \quad \text { so that } \vartheta(0)=\lim _{n \rightarrow \infty} u_{n}\left(h_{n}^{-}\right)=u\left(t_{-}\right) \text {and } \vartheta(1)=\lim _{n \rightarrow \infty} u_{n}\left(h_{n}^{+}\right)=u\left(t_{+}\right) .
\end{aligned}
$$

Therefore, applying Lemma 6.1 with the choices $m=d+1$ and, for $w=(\tau, v) \in \mathbb{R} \times \mathbb{R}^{d}$, with $\mathfrak{F}_{n}(w, \xi)=\mathfrak{F}_{n}(\tau, v, \xi):=\mathrm{b}_{\Psi_{n}}(\tau, v, \xi)$ and $\mathfrak{F}_{\infty}(w, \xi):=\mathrm{p}(\tau, v, \xi)$ (where we still 
denote by $\mathrm{b}_{\Psi_{n}}$ and by $\mathrm{p}$ their extensions to $\mathbb{R} \times \mathbb{R}^{d} \times \mathbb{R}^{d}$ by infinity), and taking into account (4.2) from Hypothesis 4.1, which ensures the validity of condition (6.1) in Lemma 6.1, we conclude

$$
\begin{aligned}
& \liminf _{n \rightarrow \infty} \int_{0}^{1} \mathrm{~b}_{\Psi_{n}}\left(\dot{\mathrm{t}}_{n}(s), \dot{\vartheta}_{n}(s),-\mathrm{D} \mathcal{E}\left(\mathrm{t}_{n}(s), \vartheta_{n}(s)\right)\right) \mathrm{d} s \\
& \geq \int_{0}^{1} \mathrm{p}(0, \dot{\vartheta}(s),-\mathrm{D} \mathcal{E}(t, \vartheta(s))) \mathrm{d} s \geq \Delta_{\mathrm{p}, \mathcal{E}}\left(t ; u\left(t_{-}\right), u\left(t_{+}\right)\right) .
\end{aligned}
$$

Similarly, we prove that

$\limsup _{n \rightarrow \infty} v_{n}\left(\left[h_{n}^{-}, t\right]\right) \geq \Delta_{\mathrm{p}, \mathcal{E}}\left(t ; u\left(t_{-}\right), u(t)\right), \quad \limsup _{n \rightarrow \infty} v_{n}\left(\left[t, h_{n}^{+}\right]\right) \geq \Delta_{\mathrm{p}, \mathcal{E}}\left(t ; u(t), u\left(t_{+}\right)\right)$.

Repeating the very same arguments as in the proof of [42, Prop. 7.3], we ultimately find that

$$
\begin{aligned}
& \liminf _{n \rightarrow \infty} \int_{s}^{t}\left(\Psi_{n}\left(\dot{u}_{n}(r)\right)+\Psi_{n}^{*}\left(-\mathrm{D} \mathcal{E}\left(r, u_{n}(r)\right)\right)\right) \mathrm{d} r \\
& \geq \operatorname{Var}_{\Psi_{0}, \mathrm{p}, \mathcal{E}}(u ;[s, t]) \text { for every } 0 \leq s \leq t \leq T,
\end{aligned}
$$

whence (5.7) also in view of (6.3). This concludes the proof.

Proof of Theorem 5.3. Let $u \in \mathrm{BV}\left([0, T] ; \mathbb{R}^{d}\right)$ be a limit point of the sequence $\left(u_{n}\right)_{n} \subset$ $\mathrm{AC}\left([0, T] ; \mathbb{R}^{d}\right)$. It follows from the lower-semicontinuity property $(5.5)$ that $\mathscr{J}_{\Psi_{0}, \mathrm{p}, \mathcal{E}}(u)=$ 0 ; hence, by Prop. $3.7 u$ is a balanced viscosity solution to $\left(\Psi_{0}, \mathrm{p}, \mathcal{E}\right)$. Moreover, for every $0 \leq s \leq t \leq T$, we have that

$$
\begin{aligned}
& \limsup _{n \rightarrow \infty} \int_{s}^{t}\left(\Psi_{n}\left(\dot{u}_{n}(r)\right)+\Psi_{n}^{*}\left(-\mathrm{D} \mathcal{E}\left(r, u_{n}(r)\right)\right)\right) \mathrm{d} r \\
& \stackrel{(1)}{\leq} \limsup _{n \rightarrow \infty}\left(\mathcal{E}\left(s, u_{n}(s)\right)-\mathcal{E}\left(t, u_{n}(t)\right)+\int_{s}^{t} \partial_{t} \mathcal{E}\left(r, u_{n}(r)\right) \mathrm{d} r+\varepsilon_{n}\right) \\
& \stackrel{(2)}{=} \mathcal{E}(s, u(s))-\mathcal{E}(t, u(t))+\int_{s}^{t} \partial_{t} \mathcal{E}(r, u(r)) \mathrm{d} r \\
& \stackrel{(3)}{=} \operatorname{Var}_{\Psi_{0}, \mathrm{p}, \mathcal{E}}(u ;[s, t])+\int_{s}^{t} \Psi_{0}^{*}(-\mathrm{D} \mathcal{E}(t, u(r))) \mathrm{d} r
\end{aligned}
$$

where (1) follows from $\mathscr{J}_{\Psi_{n}}\left(u_{n}\right) \leq \varepsilon_{n}$, (2) from convergences (5.6), and (3) from the fact that $\mathscr{J}_{\Psi_{0}, \mathrm{p}, \mathcal{E}}(u)=0$. Combining this with (5.7), we conclude the enhanced convergence properties (5.8).

Proof of Theorem 5.9. Given $u \in \mathrm{BV}\left([0, T], \mathbb{R}^{d}\right)$, we will construct a sequence $\left(u_{n}\right)_{n} \subset$ $\mathrm{AC}\left([0, T] ; \mathbb{R}^{d}\right)$ such that $u_{n} \rightarrow u$ strictly in $\mathrm{BV}\left([0, T] ; \mathbb{R}^{d}\right)$ and

$$
\limsup _{n \rightarrow \infty} \mathcal{J}_{\Psi_{n}, \mathcal{E}}\left(u_{n}\right) \leq \mathscr{J}_{\Psi_{0}, \mathrm{p}, \mathcal{E}}(u) .
$$

We split the proof of in several steps; for Steps 1-4, we suitably adapt the arguments from the proof of [12, Thm. 4.2].

Step 1: reparameterization. First, we reparameterize the curve $u$, in terms of a new time-like parameter $s$ on a domain $[0, S]$. The aim is to expand the jumps in $u$ into smooth connections. Following [41, Prop. 6.9], we define 


$$
\mathrm{s}(t):=t+\operatorname{Var}_{\Psi_{0}, \mathrm{p}, \mathcal{E}}(u ;[0, t]) .
$$

Then, there exists a Lipschitz parameterization $(\mathrm{t}, \mathrm{u}):[0, S] \rightarrow[0, T] \times \mathbb{R}$ such that $\mathrm{t}$ is non-decreasing,

$$
\mathrm{t}(\mathrm{s}(t))=t \quad \text { and } \quad \mathrm{u}(\mathrm{s}(t))=u(t) \text { for every } t \in[0, T],
$$

and such that

$$
\int_{0}^{S} \mathrm{p}\left(\dot{\mathrm{t}}(s), \dot{\mathrm{u}}(s),-\mathrm{D} \mathcal{E}(\mathrm{t}(s), \mathrm{u}(s)) \mathrm{d} s=\operatorname{Var}_{\Psi_{0}, \mathrm{p}, \mathcal{E}}(u ;[0, T])+\int_{0}^{T} \Psi_{0}^{*}(-\mathrm{D} \mathcal{E}(t, u(t))) \mathrm{d} t .\right.
$$

Moreover, it also holds that

$$
\operatorname{Var}_{\Psi_{0}}(\mathrm{u} ;[0, S])=\operatorname{Var}_{\Psi_{0}}(u ;[0, T]) .
$$

Step 2: preliminary remarks. Since we will construct a sequence $\left(u_{n}\right)_{n}$ strictly (and in particular pointwise) converging to $u$ in $\mathrm{BV}\left([0, T] ; \mathbb{R}^{d}\right)$, thanks to the smoothness of $\mathcal{E}(\mathrm{cf} .(\mathrm{E}))$, we will have for the first three contributions to $\mathcal{J}_{\Psi_{n}, \mathcal{E}}\left(u_{n}\right)$

$$
\begin{aligned}
& \mathcal{E}\left(T, u_{n}(T)\right)-\mathcal{E}\left(0, u_{n}(0)\right)-\int_{0}^{T} \partial_{t} \mathcal{E}\left(t, u_{n}(t)\right) \mathrm{d} t \\
& \quad \rightarrow \mathcal{E}(T, u(T))-\mathcal{E}(0, u(0))-\int_{0}^{T} \partial_{t} \mathcal{E}(t, u(t)) \mathrm{d} t
\end{aligned}
$$

as $n \rightarrow \infty$. Therefore, in order to prove (6.11), it will be sufficient to focus on the other terms in $\mathcal{J}_{\Psi_{n}, \mathcal{E}}$ and $\mathcal{J}_{\Psi_{0}, \mathrm{p}, \mathcal{E}}$. In view of (6.13), it will be sufficient to prove that

$$
\limsup _{n \rightarrow \infty} \int_{0}^{T}\left[\Psi_{n}\left(\dot{u}_{n}(t)\right)+\Psi_{n}^{*}\left(\mathrm{DE}\left(t, u_{n}(t)\right)\right)\right] \mathrm{d} t \leq \int_{0}^{S} \mathrm{p}(\dot{\mathrm{t}}(s), \dot{\mathrm{u}}(s), \mathrm{D} \mathcal{E}(\mathrm{t}(s), \mathrm{u}(s))) \mathrm{d} s .
$$

Step 3: definition of the new time $t_{n}$ and of the recovery sequence $u_{n}$. For the sake of simplicity, in what follows we construct a recovery sequence for a curve $u$ with jumps only at 0 and $T$, postponing to the end of the proof (cf. Step 7), the discussion of the case of a curve with countably many jumps. We define $u_{n}$ by first perturbing the time variable t: we fix $\delta>0$ and consider a selection

$$
\tau_{n}^{\delta}(s) \in \underset{\tau>0}{\operatorname{Argmin}} \mathrm{b}_{\Psi_{n}}^{\delta}(\tau, \dot{\mathrm{u}}(s),-\mathrm{D} \mathcal{E}(\mathrm{t}(s), \mathrm{u}(s)))
$$

with $b_{\Psi_{n}}^{\delta}$ given by (5.21). We define $t_{n}:[0, S] \rightarrow\left[0, T_{n}\right]$ as the solution of the differential equation

$$
\mathrm{t}_{n}(0)=0, \quad \dot{\mathrm{t}}_{n}(s)=\dot{\mathrm{t}}(s) \vee \tau_{n}^{\delta}(s) .
$$

Observe that $\dot{\mathrm{t}}(s)=0$ in $\left[0, \mathrm{~s}\left(0^{+}\right)\right] \cup\left[\mathrm{s}\left(T^{-}\right), S\right]$, but we can assume that $|\dot{\mathrm{u}}(s)|>0$ on $\left[0, \mathrm{~s}\left(0^{+}\right)\right] \cup \in\left[\mathrm{s}\left(T^{-}\right), S\right]$. This will be sufficient to guarantee that $\operatorname{Argmin}_{\tau>0} \mathrm{~b}_{\Psi_{n}}^{\delta}(\tau, \dot{\mathrm{u}}(s)$, $\mathrm{DE}(\mathrm{t}(s), \mathrm{u}(s))) \neq \emptyset$ for $s \in\left[0, \mathrm{~s}\left(0^{+}\right)\right] \cup \in\left[\mathrm{s}\left(T^{-}\right), S\right]$. Thus, $\tau_{n}^{\delta}$ shall be well defined on the latter set. On the other hand, for $s \in\left[\mathrm{s}\left(0^{+}\right), \mathrm{s}\left(T^{-}\right)\right]$, we have $\dot{\mathrm{t}}(s)=\left.\frac{1}{\dot{\mathrm{s}(t)}}\right|_{t=\mathrm{t}(s)}>0$. All in all, $\dot{\mathrm{t}}_{n}(s)>0$ for all $s \in[0, S]$. The range of $\mathrm{t}_{n}$ is $\left[0, T_{n}\right]$, with $T_{n} \geq T$; since the recovery sequence $u_{n}$ has to be defined on the interval $[0, T]$, we rescale $t_{n}$ by 


$$
\lambda_{n}:=\frac{T_{n}}{T} \geq 1,
$$

and define our recovery sequence as follows:

$$
u_{n}(t):=\mathrm{u}\left(\mathrm{t}_{n}^{-1}\left(t \lambda_{n}\right)\right), \quad \text { so that } \quad \dot{u}_{n}(t)=\frac{\dot{\mathrm{u}}}{\dot{\mathrm{t}}_{n}}\left(\mathrm{t}_{n}^{-1}\left(t \lambda_{n}\right)\right) \lambda_{n} .
$$

Now we substitute the explicit formula for $u_{n}$; we perform a change of variable and obtain

$$
\begin{aligned}
& \int_{0}^{T}\left(\Psi_{n}\left(\dot{u}_{n}(t)\right)+\Psi_{n}^{*}\left(t,-\mathrm{D} \mathcal{E}\left(t, u_{n}(t)\right)\right)\right) \mathrm{d} t \\
& \quad=\int_{0}^{T}\left(\Psi_{n}\left(\frac{\dot{\mathrm{u}}}{\dot{\mathrm{t}}_{n}}\left(\mathrm{t}_{n}^{-1}\left(t \lambda_{n}\right)\right) \lambda_{n}\right)+\Psi_{n}^{*}\left(-\mathrm{D} \mathcal{E}\left(t, \mathrm{u}\left(\mathrm{t}_{n}^{-1}\left(t \lambda_{n}\right)\right)\right)\right)\right) \mathrm{d} t \\
& \quad=\int_{0}^{S}\left(\Psi_{n}\left(\frac{\dot{\mathrm{u}}(s)}{\dot{\mathrm{t}}_{n}(s)} \lambda_{n}\right)+\Psi_{n}^{*}\left(-\mathrm{D} \mathcal{E}\left(\mathrm{t}_{n}(s) \lambda_{n}^{-1}, \mathrm{u}(s)\right)\right)\right) \frac{\dot{\mathrm{t}}_{n}(s)}{\lambda_{n}} \mathrm{~d} s,
\end{aligned}
$$

so that

$$
\begin{aligned}
& \int_{0}^{T}\left(\Psi_{n}\left(\dot{u}_{n}(t)\right)+\Psi_{n}^{*}\left(-\mathrm{D} \mathcal{E}\left(t, u_{n}(t)\right)\right)\right) \mathrm{d} t \\
& \quad=\int_{0}^{S} \mathrm{~b}_{\Psi_{n}}\left(\lambda_{n}^{-1} \dot{\mathrm{t}}_{n}(s), \dot{\mathrm{u}}(s),-\mathrm{D} \mathcal{E}\left(\mathrm{t}_{n}(s) \lambda_{n}^{-1}, \mathrm{u}(s)\right)\right) \mathrm{d} s .
\end{aligned}
$$

Step 4: Strict convergence of $\left(u_{n}\right)_{n}$. Recall that we need to prove the pointwise convergence $u_{n}(t) \rightarrow u(t)$ for all $t \in[0, T]$ and the convergence of the variations. For this, it will be crucial to have the following property that shall be verified (even uniformly w.r.t. $s \in[0, S]$ ) both in the stochastic (cf. (6.24)) and in the vanishing-viscosity cases (cf. (6.39)):

$$
\tau_{n}^{\delta}(s) \rightarrow 0 \text { as } n \rightarrow \infty \text { for a.a. } s \in(0, S) .
$$

This shall imply that $\dot{\mathrm{t}}_{n}(s) \rightarrow \dot{\mathrm{t}}(s)$ for almost all $s \in(0, S)$, and then it will hold

$$
\begin{aligned}
& \mathrm{t}_{n}(s) \rightarrow \mathrm{t}(s) \quad \text { for every } s \in[0, S] \\
& \quad \Longrightarrow \quad \lambda_{n} \rightarrow 1, \quad \mathrm{t}_{n}^{-1}\left(t \lambda_{n}\right) \rightarrow \mathrm{s}(t) \text { for every } t \in[0, T] .
\end{aligned}
$$

Moreover, $\dot{\mathrm{t}}_{n}(s)>0$ implies that $\mathrm{t}_{n}^{-1}(0)=0$ and $\mathrm{t}_{n}^{-1}\left(T_{n}\right)=S$, and so we will have the desired pointwise convergence

$$
u_{n}(t)=\mathrm{u}\left(\mathrm{t}_{n}^{-1}\left(t \lambda_{n}\right)\right) \rightarrow \mathrm{u}(\mathrm{s}(t)) \stackrel{(6.12)}{=} u(t) \quad \text { for every } t \in[0, T] .
$$

The convergence of the variations will be automatic, since by definition of $u_{n}$ we will have

$$
\int_{0}^{T} A\left\|\dot{u}_{n}(t)\right\|_{1} \mathrm{~d} t=\int_{0}^{S} A\|\dot{\mathrm{u}}(s)\|_{1} \mathrm{~d} s=\operatorname{Var}_{\Psi_{0}}(\mathrm{u} ;[0, S]) \stackrel{(6.14)}{=} \operatorname{Var}_{\Psi_{0}}(u ;[0, T]) .
$$

In view of the above observations, from now on we can concentrate on the proof of the lim sup estimate (6.15).

Step 5: strategy for (6.15). First of all, we will show the following pointwise lim supinequality

$$
\begin{aligned}
& \limsup _{n \rightarrow \infty} \mathrm{b}_{\Psi_{n}}\left(\lambda_{n}^{-1} \dot{\mathrm{t}}_{n}(s), \dot{\mathrm{u}}(s),-\mathrm{D} \mathcal{E}\left(\mathrm{t}_{n}(s) \lambda_{n}^{-1}, \mathrm{u}(s)\right)\right) \\
& \quad \leq \mathrm{p}(\dot{\mathrm{t}}(s), \dot{\mathrm{u}}(s),-\mathrm{D} \mathcal{E}(\mathrm{t}(s), \mathrm{u}(s)) \text { for a.a. } s \in(0, S) .
\end{aligned}
$$


Secondly, we will apply the following version of the Fatou's lemma

$$
\left.\begin{array}{rr}
\lim \sup _{n \rightarrow \infty} f_{n}(s) \leq f(s) \text { for a.a. } s \in(0, S), \\
f_{n}(s) \leq g_{n}(s) & \text { for a.a. } s \in(0, S), \\
g_{n} \rightarrow g & \text { in } L^{1}(0, S),
\end{array}\right\} \Longrightarrow \limsup _{n \rightarrow \infty} \int_{0}^{S} f_{n}(s) \mathrm{d} s \leq \int_{0}^{S} f(s) \mathrm{d} s,
$$

for measurable functions $\left(f_{n}\right)_{n}$ and $f$, in order to conclude that

$$
\begin{aligned}
& \underset{n \rightarrow \infty}{\limsup } \int_{0}^{S} \mathrm{~b}_{\Psi_{n}}\left(\lambda_{n}^{-1} \dot{\mathrm{t}}_{n}(s), \dot{\mathrm{u}}(s),-\mathrm{DE}\left(\mathrm{t}_{n}(s) \lambda_{n}^{-1}, \mathrm{u}(s)\right)\right) \mathrm{d} s \\
& \quad \leq \int_{0}^{S} \mathrm{p}(\dot{\mathrm{t}}(s), \dot{\mathrm{u}}(s),-\mathrm{DE}(\mathrm{t}(s), \mathrm{u}(s))) \mathrm{d} s,
\end{aligned}
$$

whence (6.15) and ultimately (6.11). For the proof of (6.21) and (6.23), we will distinguish the stochastic and the vanishing-viscosity cases.

Step 6a: proof of (6.21) and (6.23) for $\Psi_{n}$ given by (5.18) (stochastic approximation). Preliminarily, we observe that, with the very same calculations as for (5.30) (cf. Claim 3.4 in the proof of Proposition 5.8), one has

$$
\begin{aligned}
& \tau_{n}^{\delta}(s) \leq \sqrt{\frac{d^{2}\|\dot{u}(s)\|_{\infty}^{2}}{n^{2} \delta^{2}+n^{2}\left(\Psi_{n}^{*}(-\mathrm{DE}(\mathrm{t}(s), \mathrm{u}(s)))\right)^{2}}} \rightarrow 0 \text { for almost all } s \in(0, S), \quad \text { and thus } \\
& \sup _{s \in[0, S]} \tau_{n}^{\delta}(s) \leq \frac{C}{\delta n} \rightarrow 0 \quad \text { as } n \rightarrow \infty,
\end{aligned}
$$

(with a slight abuse of notation, we use the symbol sup also for an essential supremum) where we have exploited the Lipschitz continuity of $u$. In order to prove the pointwise estimate (6.21), we start with the following algebraic manipulation

$$
\begin{aligned}
\mathrm{b}_{\Psi_{n}} & \left(\lambda_{n}^{-1} \dot{\mathrm{t}}_{n}(s), \dot{\mathrm{u}}(s),-\mathrm{D} \mathcal{E}\left(\mathrm{t}_{n}(s) \lambda_{n}^{-1}, \mathrm{u}(s)\right)\right) \\
= & \mathrm{b}_{\Psi_{n}}^{\delta}\left(\tau_{n}^{\delta}(s), \dot{\mathrm{u}}(s),-\mathrm{D} \mathcal{E}(\mathrm{t}(s), \mathrm{t}(s))\right)-\tau_{n}^{\delta}(s) \delta \\
& +\dot{\mathrm{t}}_{n}(s) \Psi_{n}^{*}\left(-\mathrm{D} \mathcal{E}\left(\mathrm{t}_{n}(s) \lambda_{n}^{-1}, \mathrm{u}(s)\right)\right)-\tau_{n}^{\delta}(s) \Psi_{n}^{*}(-\mathrm{D} \mathcal{E}(\mathrm{t}(s), \mathrm{u}(s))) \\
& +\frac{\dot{\mathrm{t}}_{n}(s)}{\lambda_{n}} \Psi_{n}\left(\frac{\dot{\mathrm{u}}(s)}{\dot{\mathrm{t}}_{n}(s)} \lambda_{n}\right)-\tau_{n}^{\delta}(s) \Psi_{n}\left(\frac{\dot{\mathrm{u}}(s)}{\tau_{n}^{\delta}(s)}\right)
\end{aligned}
$$

and prove the following three claims for the terms on the right-hand side.

Claim 6.a.1: there holds

$$
\begin{aligned}
\limsup _{n \rightarrow \infty} & \mathrm{b}_{\Psi_{n}}^{\delta}\left(\tau_{n}^{\delta}(s), \dot{\mathrm{u}}(s),-\mathrm{D} \mathcal{E}(\mathrm{t}(s), \mathrm{u}(s))\right)-\tau_{n}^{\delta}(s) \delta \\
\leq & \mathrm{p}(\dot{\mathrm{t}}(s), \dot{\mathrm{u}}(s),-\mathrm{D} \mathcal{E}(\mathrm{t}(s), \mathrm{u}(s))) \quad \text { for a.a. } s \in(0, S),
\end{aligned}
$$

with $\mathrm{p}$ given by (5.20).

Indeed, the representation formula (5.22) for $\min _{\tau>0} \mathrm{~b}_{\Psi_{n}}^{\delta}\left(\tau_{n}^{\delta}(s), \dot{\mathrm{u}}(s),-\mathrm{D} \mathcal{E}(\mathrm{t}(s), \mathrm{u}(s))\right)$ and estimate (5.27) (cf. Claim 3.3 in the proof of Proposition 5.8) yield

$$
\begin{aligned}
\mathrm{b}_{\Psi_{n}}^{\delta}\left(\tau_{n}^{\delta}(s), \dot{\mathrm{u}}(s),-\mathrm{DE}(\mathrm{t}(s), \mathrm{u}(s))\right)= & \sup \left\{\langle\xi, \dot{\mathrm{u}}(s)\rangle \mid \xi \in K_{n, \delta}^{*}(-\mathrm{DE}(\mathrm{t}(s), \mathrm{u}(s)))\right\} \\
\leq & \sup \left\{\|\dot{\mathrm{u}}(s)\|_{1}\|\xi\|_{\infty} \mid \xi \in K_{n, \delta}^{*}(-\mathrm{DE}(\mathrm{t}(s), \mathrm{u}(s)))\right\} \\
\leq & \|\dot{\mathrm{u}}(s)\|_{1}\left(A \vee\|\mathrm{DE}(\mathrm{t}(s), \mathrm{u}(s))\|_{\infty}\right) \\
& +\frac{1}{n}\|\dot{\mathrm{u}}(s)\|_{1} \log (2 e n \delta),
\end{aligned}
$$


and we conclude sending $n \rightarrow \infty$. Furthermore, we observe that $\tau_{n}^{\delta}(s) \delta \rightarrow 0$ as $n \rightarrow \infty$ thanks to the previously proved (6.24).

Claim 6.a.2: there holds

$\limsup _{n \rightarrow \infty}\left(\dot{\mathrm{t}}_{n}(s) \Psi_{n}^{*}\left(-\mathrm{D} \mathcal{E}\left(\mathrm{t}_{n}(s) \lambda_{n}^{-1}, \mathrm{u}(s)\right)\right)-\tau_{n}^{\delta}(s) \Psi_{n}^{*}(-\mathrm{D} \mathcal{E}(\mathrm{t}(s), \mathrm{u}(s)))\right) \leq 0$ for a.a. $s \in(0, S)$.

Indeed, from the assumed uniform Lipschitz continuity of $\mathrm{DE}(\cdot, u)(\mathrm{cf} .(5.35))$, we gather that

$$
\begin{aligned}
\left|\mathrm{D}_{i} \mathcal{E}\left(\mathrm{t}_{n}(s) \lambda_{n}^{-1}, \mathrm{u}(s)\right)\right|-\left|\mathrm{D}_{i} \mathcal{E}(\mathrm{t}(s), \mathrm{u}(s))\right| \leq & \left|\mathrm{D}_{i} \mathcal{E}\left(\mathrm{t}_{n}(s) \lambda_{n}^{-1}, \mathrm{u}(s)\right)-\mathrm{D}_{i} \mathcal{E}(\mathrm{t}(s), \mathrm{u}(s))\right| \\
& \leq L_{\mathrm{E}}\left|\mathrm{t}_{n}(s) \lambda_{n}^{-1}-\mathrm{t}(s)\right|
\end{aligned}
$$

for all $s \in[0, S]$ and all $i=1, \ldots, d$. We now observe that

$$
\begin{aligned}
\left|\mathrm{t}_{n}(s) \lambda_{n}^{-1}-\mathrm{t}(s)\right| & \leq\left|\mathrm{t}_{n}(s) \lambda_{n}^{-1}-\mathrm{t}(s) \lambda_{n}^{-1}+\mathrm{t}(s) \lambda_{n}^{-1}-\mathrm{t}(s)\right| \\
& \leq \mathrm{t}(s)\left(1-\lambda_{n}^{-1}\right)+\lambda_{n}^{-1}\left|\mathrm{t}_{n}(s)-\mathrm{t}(s)\right| \\
& \leq T\left(1-\frac{1}{\lambda_{n}}\right)+\lambda_{n}^{-1} \int_{0}^{s}\left(\left(\dot{\mathrm{t}}(r) \vee \tau_{n}^{\delta}(r)\right)-\dot{\mathrm{t}}(r)\right) \mathrm{d} r \\
& \leq T\left(1-\frac{1}{\lambda_{n}}\right)+\int_{0}^{s} \tau_{n}^{\delta}(r) \mathrm{d} r
\end{aligned}
$$

where we have used the fact that $\lambda_{n} \geq 1$ and the definition of $t_{n}$ from (6.17). We also have

$$
\begin{aligned}
T\left(1-\lambda_{n}^{-1}\right)=\frac{T}{T_{n}}\left(T_{n}-T\right) \leq & \left(\int_{0}^{\mathrm{s}\left(0^{+}\right)} \tau_{n}^{\delta}(r) \mathrm{d} r+\int_{\mathrm{s}\left(0^{+}\right)}^{\mathrm{s}\left(T^{-}\right)}\left(\left(\dot{\mathrm{t}}(r) \vee \tau_{n}^{\delta}(r)\right)\right.\right. \\
& \left.-\dot{\mathrm{t}}(r)) d r+\int_{\mathrm{s}\left(T^{-}\right)}^{S} \tau_{n}^{\delta}(r) \mathrm{d} r\right) \\
\leq & \left(\int_{0}^{S} \tau_{n}^{\delta}(r) \mathrm{d} r\right) \leq\left(\sup _{s \in[0, S]} \tau_{n}^{\delta}(s)\right) S
\end{aligned}
$$

again using Definition (6.17) of $t_{n}$. Hence, combining estimate (6.29) with (6.30) and (6.31), we gather that

$$
\begin{aligned}
& \left|\mathrm{D}_{i} \mathcal{E}\left(\mathrm{t}_{n}(s) \lambda_{n}^{-1}, \mathrm{u}(s)\right)\right|-\left|\mathrm{D}_{i} \mathcal{E}(\mathrm{t}(s), \mathrm{u}(s))\right| \\
& \leq C \sup _{s \in[0, S]} \tau_{n}^{\delta}(s) \doteq \bar{C}(n) \text { for all } s \in[0, S], \quad \text { with } \\
& \quad \sup _{n \in \mathbb{N}} n \bar{C}(n) \doteq \bar{C}<\infty,
\end{aligned}
$$

the latter estimate due to (6.24). Therefore, using now the explicit formula (2.7) for $\Psi_{n}^{*}$ we get for almost all $s \in(0, S)$ that

$$
\begin{aligned}
& \dot{\mathrm{t}}_{n}(s) \Psi_{n}^{*}\left(-\mathrm{D} \mathcal{E}\left(\mathrm{t}_{n}(s) \lambda_{n}^{-1}, \mathrm{u}(s)\right)\right)-\tau_{n}^{\delta}(s) \Psi_{n}^{*}(-\mathrm{D} \mathcal{E}(\mathrm{t}(s), \mathrm{u}(s))) \\
& \stackrel{(1)}{\leq} \frac{\dot{\mathrm{t}}_{n}(s)}{n} e^{-n A} \sum_{i=1}^{d} \cosh \left(n\left|\mathrm{D}_{i} \mathcal{E}\left(\mathrm{t}_{n}(s) \lambda_{n}^{-1}, \mathrm{u}(s)\right)\right|\right) \\
& \stackrel{(2)}{\leq} \frac{\dot{\mathrm{t}}_{n}(s)}{n} e^{-n A} \sum_{i=1}^{d} \cosh \left(n\left|\mathrm{D}_{i} \mathcal{E}(\mathrm{t}(s), \mathrm{u}(s))\right|+n \bar{C}(n)\right)
\end{aligned}
$$




$$
\begin{aligned}
& \stackrel{\text { (3) }}{\leq} \frac{d \dot{\mathrm{t}}_{n}(s) e^{-n A}}{2 n}+\frac{\dot{\mathrm{t}}_{n}(s)}{2 n} e^{\bar{C}} e^{-n A} \sum_{i=1}^{d} e^{n\left|\mathrm{D}_{i} \mathcal{E}(\mathrm{t}(s), \mathrm{u}(s))\right|} \\
& \stackrel{(4))}{\leq} \begin{cases}\frac{d}{n} \dot{\mathrm{t}}_{n}(s)\left(1+e^{\bar{C}}\right) \doteq \frac{C_{1}}{n} & \text { for } s \in\left[\mathrm{s}\left(0^{+}\right), \mathrm{s}\left(T^{-}\right)\right], \\
C_{2}\left(\frac{1}{n}+\sup _{s \in[0, S]} \tau_{n}^{\delta}(s) \Psi_{n}^{*}(-\mathrm{D} \mathcal{E}(\mathrm{t}(s), \mathrm{u}(s)))\right) & \text { for } s \in\left[0, \mathrm{~s}\left(0^{+}\right)\right) \cup\left(\mathrm{s}\left(T^{-}\right), S\right],\end{cases}
\end{aligned}
$$

where (1) follows from the positivity of $\Psi_{n}^{*}$ and from the trivial inequality $\cosh (n x)-1 \leq$ $\cosh (n|x|)$, (2) from (6.29), (3) from (6.32) and using the estimate $\cosh (x) \leq \frac{e^{x}+1}{2}$ for all $x \geq 0$, and (4) is due to the fact that $\|\mathrm{DE}(\mathrm{t}(s), \mathrm{u}(s))\|_{\infty} \leq A$ for $s \in\left[\mathrm{s}\left(0^{+}\right), \mathrm{s}\left(T^{-}\right)\right]$, and to an elementary inequality on $\left[0, \mathrm{~s}\left(0^{+}\right)\right) \cup\left(\mathrm{s}\left(T^{-}\right), S\right]$. Clearly, $\frac{C_{1}}{n} \rightarrow 0$; on the other hand, it follows again from (6.24) and the Lipschitz continuity of $u$ that

$$
\begin{aligned}
\sup _{s \in[0, S]} \tau_{n}^{\delta}(s) \Psi_{n}^{*}(-\mathrm{D} \mathcal{E}(\mathrm{t}(s), \mathrm{u}(s))) & \leq \sup _{s \in[0, S]} \frac{d\|\dot{u}(s)\|_{\infty} \Psi_{n}^{*}(-\mathrm{D} \mathcal{E}(\mathrm{t}(s), \mathrm{u}(s)))}{n\left(\Psi_{n}^{*}(-\mathrm{D} \mathcal{E}(\mathrm{t}(s), \mathrm{u}(s)))\right)} \\
& \leq \frac{C_{3}}{n} \rightarrow 0 \text { as } n \rightarrow \infty
\end{aligned}
$$

Therefore, (6.28) ensues.

\section{Claim 6.a.3: there holds}

$$
\limsup _{n \rightarrow \infty}\left(\frac{\dot{\mathrm{t}}_{n}(s)}{\lambda_{n}} \Psi_{n}\left(\frac{\dot{\mathrm{u}}(s)}{\dot{\mathrm{t}}_{n}(s)} \lambda_{n}\right)-\tau_{n}^{\delta}(s) \Psi_{n}\left(\frac{\dot{\mathrm{u}}(s)}{\tau_{n}^{\delta}(s)}\right)\right) \leq 0 \quad \text { for a.a. } s \in(0, S) .
$$

We use the explicit formula (5.18) for $\Psi_{n}$, obtaining

$$
\begin{aligned}
& \frac{\dot{\mathrm{t}}_{n}(s)}{\lambda_{n}} \Psi_{n}\left(\frac{\dot{\mathrm{u}}(s)}{\dot{\mathrm{t}}_{n}(s)} \lambda_{n}\right) \\
& \stackrel{\dot{\mathrm{t}}_{n} \geq \tau_{n}^{\delta}}{\leq} \frac{\tau_{n}^{\delta}(s)}{\lambda_{n}} \Psi_{n}\left(\frac{\dot{\mathrm{u}}(s)}{\tau_{n}^{\delta}(s)} \lambda_{n}\right) \\
& \leq \frac{d \tau_{n}^{\delta}(s) e^{-n A}}{n}+\sum_{i=1}^{d}\left[\frac{\dot{\mathrm{u}}_{i}(s)}{n} \log \left(\lambda_{n} \frac{\frac{\dot{\mathrm{u}}_{i}(s)}{\tau_{n}^{\delta}(s)}+\sqrt{\left(\frac{\dot{\mathrm{u}}_{i}(s)}{\tau_{n}^{\delta}(s)}\right)^{2}+\frac{e^{-2 n A}}{\lambda_{n}^{2}}}}{e^{-n A}}\right)\right. \\
& \left.-\frac{1}{n} \sqrt{\dot{\mathrm{u}}_{i}(s)^{2}+\left(\frac{\tau_{n}^{\delta}(s) e^{-n A}}{\lambda_{n}}\right)^{2}}\right] \\
& \stackrel{\lambda_{n} \geq 1}{\leq} \tau_{n}^{\delta}(s) \Psi_{n}\left(\frac{\dot{\mathrm{u}}(s)}{\tau_{n}^{\delta}(s)}\right)+\sum_{i=1}^{d}\left[\frac{\dot{\mathrm{u}}_{i}(s)}{n} \log \left(\lambda_{n}\right)-\frac{1}{n} \sqrt{\dot{\mathrm{u}}_{i}(s)^{2}+\left(\frac{\tau_{n}^{\delta}(s) e^{-n A}}{\lambda_{n}}\right)^{2}}\right. \\
& \left.+\frac{1}{n} \sqrt{\dot{\mathrm{u}}_{i}(s)^{2}+\left(\tau_{n}^{\delta}(s) e^{-n A}\right)^{2}}\right],
\end{aligned}
$$

for almost all $s \in(0, S)$, whence 


$$
\begin{aligned}
& \dot{\mathrm{t}}_{n}(s) \frac{1}{\lambda_{n}} \Psi_{n}\left(\frac{\dot{\mathrm{u}}(s)}{\dot{\mathrm{t}}_{n}(s)} \lambda_{n}\right)-\tau_{n}^{\delta}(s) \Psi_{n}\left(\frac{\dot{\mathrm{u}}(s)}{\tau_{n}^{\delta}(s)}\right) \\
& \quad \leq \sum_{i=1}^{d}\left(\frac{\dot{\mathrm{u}}_{i}(s)}{n} \log \left(\lambda_{n}\right)-\frac{1}{n} \sqrt{\left(\dot{\mathrm{u}}_{i}(s)\right)^{2}+\left(\frac{\tau_{n}^{\delta}(s) e^{-n A}}{\lambda_{n}}\right)^{2}}+\frac{1}{n} \sqrt{\left(\dot{\mathrm{u}}_{i}(s)\right)^{2}+\left(\tau_{n}^{\delta}(s) e^{-n A}\right)^{2}}\right) .
\end{aligned}
$$

Observe that the right-hand side of (6.36) tends to zero as $n \rightarrow \infty$ taking into account that $\sup _{s \in[0, S]}\|\dot{\mathrm{u}}(s)\|_{\infty} \leq C$, that $\lambda_{n} \rightarrow 1$, and that $\sup _{s \in[0, S]} \tau_{n}^{\delta}(s) \rightarrow 0$ by (6.24). This yields (6.35) and, ultimately, (6.21).

Finally, we conclude the integrated lim sup-estimate (6.23) by observing that the Fatou's lemma (cf. (6.22)) applies: this can be checked combining (6.24), (6.25), (6.27) (taking into account that $\left.\sup _{s \in[0, S]}\|\dot{u}(s)\|_{1} \leq C\right),(6.33),(6.34)$, and (6.36).

Step 6b: proof of (6.21) and (6.23) for $\Psi_{n}$ given by (5.33) (vanishing-viscosity approximation).

To simplify the notation, in what follows we shall focus on the particular case in which the sequence $\left(\varepsilon_{n}\right)_{n}$ is given by

$$
\varepsilon_{n}=\frac{1}{n}
$$

Preliminarily, we recall that, in the case (5.33),

$$
\Psi_{n}^{*}(\xi)=\frac{1}{2 \varepsilon_{n}}\left(\min _{\zeta \in K^{*}}\|\xi-\zeta\|_{*}\right)^{2}=\frac{n}{2} d_{*}\left(\xi, K^{*}\right)^{2},
$$

where $\|\cdot\|_{*}$ is the dual norm to $\|\cdot\|$, and $d_{*}\left(\cdot, K^{*}\right)$ denotes the induced distance from the set $K^{*}$. Taking into account (6.37), we provide a bound for the parameter $\tau_{n}^{\delta}$ from (6.16) again resorting to the Euler-Lagrange equation (5.29). In the present case, it rewrites as

$A\left\|\frac{\dot{\mathrm{u}}(s)}{\tau_{n}^{\delta}(s)}\right\|_{1}-\frac{1}{2 n}\left\|\frac{\dot{\mathrm{u}}(s)}{\tau_{n}^{\delta}(s)}\right\|^{2}-\left\langle\partial \Psi_{0}\left(\frac{\dot{\mathrm{u}}(s)}{\tau_{n}^{\delta}(s)}\right), \frac{\dot{\mathrm{u}}(s)}{\tau_{n}^{\delta}(s)}\right\rangle+\frac{n}{2} d_{*}\left(-\mathrm{D} \mathcal{E}(\mathrm{t}(s), \mathrm{u}(s)), K^{*}\right)^{2}+\delta=0$,

where we have formally written $\partial \Psi_{0}$ as a single-valued mapping. Taking into account that $\left\langle\partial \Psi_{0}(\zeta), \zeta\right\rangle=\Psi_{0}(\zeta)$ for all $\zeta \in \mathbb{R}^{d}$, we ultimately conclude that

$$
\tau_{n}^{\delta}(s)=\frac{\|\dot{\mathrm{u}}(s)\|}{\sqrt{2 n \delta+n^{2} d_{*}\left(-\mathrm{D} \mathcal{E}(\mathrm{t}(s), \mathrm{u}(s)), K^{*}\right)^{2}}} \leq \frac{\|\dot{\mathrm{u}}(s)\|}{\sqrt{2 n \delta}} \quad \text { for a.a. } s \in(0, S) .
$$

In what follows, we will take

$$
\delta=\delta_{n} \text { such that } \delta_{n} \rightarrow \infty \text { and } \delta_{n} \frac{1}{n} \rightarrow 0 \text { as } n \rightarrow \infty
$$

but we will continue to write $\delta$ in place of $\delta_{n}$ for shorter notation. 
In order to show (6.21), we start from the very same algebraic manipulation as in (6.25) and prove that the terms on the right-hand side converge to the desired limit. We observe that

$$
\begin{aligned}
& \mathrm{b}_{\Psi_{n}}^{\delta}\left(\tau_{n}^{\delta}(s), \dot{\mathrm{u}}(s),-\mathrm{D} \mathcal{E}(\mathrm{t}(s), \mathrm{u}(s))\right) \\
& =\tau_{n}^{\delta}(s) \Psi_{n}\left(\frac{\dot{\mathrm{u}}(s)}{\tau_{n}^{\delta}(s)}\right)+\tau_{n}^{\delta}(s) \Psi_{n}^{*}(-\mathrm{D} \mathcal{E}(\mathrm{t}(s), \mathrm{u}(s)))+\tau_{n}^{\delta}(s) \delta \\
& \quad \stackrel{(6.39)}{=} \Psi_{0}(\dot{\mathrm{u}}(s))+\frac{\|\dot{\mathrm{u}}(s)\|}{2 n} \sqrt{2 n \delta+n^{2} d_{*}\left(-\mathrm{D} \mathcal{E}(\mathrm{t}(s), \mathrm{u}(s)), K^{*}\right)^{2}} \\
& \quad+\frac{n\|\dot{\mathrm{u}}(s)\| d_{*}\left(-\mathrm{D} \mathcal{E}(\mathrm{t}(s), \mathrm{u}(s)), K^{*}\right)^{2}}{2 \sqrt{2 n \delta+n^{2} d_{*}\left(-\mathrm{D} \mathcal{E}(\mathrm{t}(s), \mathrm{u}(s)), K^{*}\right)^{2}}}+\tau_{n}^{\delta}(s) \delta \\
& \quad \stackrel{n \rightarrow \infty}{\rightarrow} \Psi_{0}(\dot{\mathrm{u}}(s))+\|\dot{\mathrm{u}}(s)\| d_{*}\left(-\mathrm{D} \mathcal{E}(\mathrm{t}(s), \mathrm{u}(s)), K^{*}\right) \\
& \leq \mathrm{p}(\dot{\mathrm{t}}(s), \dot{\mathrm{u}}(s),-\mathrm{D} \mathcal{E}(\mathrm{t}(s), \mathrm{u}(s))),
\end{aligned}
$$

where the last inequality follows from the fact that $\mathrm{D} \mathcal{E}(\mathrm{t}(s), \mathrm{u}(s)) \in K^{*}$ when $\dot{\mathrm{t}}(s)>0$. Thus, we conclude the analogue of (6.26). Moreover, observe that, as a consequence of estimate (6.39) and of the scaling for $\delta_{n}$ from (6.40), we have

$$
\delta \sup _{s \in[0, S]} \tau_{n}^{\delta}(s) \rightarrow 0 \text { as } n \rightarrow \infty .
$$

We then proceed to show the counterpart to (6.28). The very same calculations as in (6.29) (cf. also (6.31)), and again (6.39) give for every $s \in[0, S]$ and all $i=1, \ldots, d$

$$
\left|\mathrm{D}_{i} \mathcal{E}\left(\mathrm{t}_{n}(s) \lambda_{n}^{-1}, \mathrm{u}(s)\right)-\mathrm{D}_{i} \mathcal{E}(\mathrm{t}(s), \mathrm{u}(s))\right| \leq C \sup _{s \in[0, S]} \tau_{n}^{\delta}(s) \leq \frac{C}{\sqrt{n \delta}} .
$$

Resorting now to the explicit formula (6.37) for $\Psi_{n}^{*}$ (and using $\xi_{n}(s)$ and $\xi(s)$ as placeholders for $-\mathrm{D} \mathcal{E}\left(\mathrm{t}_{n}(s) \lambda_{n}^{-1}, \mathrm{u}(s)\right)$ and $-\mathrm{D} \mathcal{E}(\mathrm{t}(s), \mathrm{u}(s))$, respectively, to avoid overburdening notation), we get

$$
\begin{aligned}
\dot{\mathrm{t}}_{n}(s) \Psi_{n}^{*}\left(\xi_{n}(s)\right)-\tau_{n}^{\delta}(s) \Psi_{n}^{*}(\xi(s)) & \stackrel{(1)}{=} \dot{\mathrm{t}}_{n}(s) \frac{n}{2} d_{*}\left(\xi_{n}(s), K^{*}\right)^{2} \\
& \stackrel{(2)}{\leq} \dot{\mathrm{t}}_{n}(s) \frac{n}{2}\left\|\xi_{n}(s)-\xi(s)\right\|_{*}^{2} \\
& \stackrel{(3)}{\leq} \frac{C}{\delta} \quad \text { for a.a. } s \in\left(\mathrm{s}\left(0^{+}\right), \mathrm{s}\left(T^{-}\right)\right) .
\end{aligned}
$$

In (6.44), (1) and (2) are due to the fact that $\mathrm{DE}(\mathrm{t}(s), \mathrm{u}(s)) \in K^{*}$ for almost all $s \in$ $\left(\mathrm{s}\left(0^{+}\right), \mathrm{s}\left(T^{-}\right)\right)$, so that $\Psi_{n}^{*}(\xi(s))=0$, and (3) follows from estimate (6.43). To prove the analogue of (6.28), we will first treat the case in which $s \in\left[0, \mathrm{~s}\left(0^{+}\right)\right) \cup\left(\mathrm{s}\left(T^{-}\right), S\right]$ (where $\dot{\mathrm{t}}_{n}(s)=\tau_{n}^{\delta}(s)$ ). Here, we use the Lipschitz estimate (6.43) and the explicit formula for $\Psi_{n}^{*}$. Thus, we find

$$
\begin{aligned}
& \tau_{n}^{\delta}(s)\left(\Psi_{n}^{*}\left(\xi_{n}(s)\right)-\Psi_{n}^{*}(\xi(s))\right) \\
& =\frac{n}{2} \tau_{n}^{\delta}(s)\left(d_{*}\left(\xi_{n}(s), K^{*}\right)^{2}-d_{*}\left(\xi(s), K^{*}\right)^{2}\right) \\
& \leq \frac{n}{2} \tau_{n}^{\delta}(s)\left(\left(d_{*}\left(\xi_{n}(s), \xi(s)\right)+d_{*}\left(\xi(s), K^{*}\right)\right)^{2}-d_{*}\left(\xi(s), K^{*}\right)^{2}\right) \\
& \leq \frac{n}{2} \tau_{n}^{\delta}(s)\left(d_{*}\left(\xi_{n}(s), \xi(s)\right)^{2}+2 d_{*}\left(\xi(s), K^{*}\right) d_{*}\left(\xi_{n}(s), \xi(s)\right)\right) \\
& \stackrel{(6.43)}{\leq} n \tau_{n}^{\delta}(s)\left(\frac{C}{n \delta}+\frac{C}{\sqrt{n \delta}}\right) \quad \text { for all } s \in\left[0, \mathrm{~s}\left(0^{+}\right)\right) \cup\left(\mathrm{s}\left(T^{-}\right), S\right]
\end{aligned}
$$


Combining (6.44) and (6.45) we infer (6.28), since $\delta=\delta_{n} \rightarrow \infty$ and $\frac{\delta_{n}}{n} \rightarrow 0$ as $n \rightarrow \infty$. In order to prove the analogue of (6.35), we use the explicit formula (5.33) of $\Psi_{n}$, obtaining

$$
\begin{aligned}
\frac{\dot{\mathrm{t}}_{n}(s)}{\lambda_{n}} \Psi_{n}\left(\frac{\dot{\mathrm{u}}(s)}{\dot{\mathrm{t}}_{n}(s)} \lambda_{n}\right) & \stackrel{\dot{\mathrm{t}}_{n} \geq \tau_{n}^{\delta}}{\leq} \frac{\tau_{n}^{\delta}(s)}{\lambda_{n}} \Psi_{n}\left(\frac{\dot{\mathrm{u}}(s)}{\tau_{n}^{\delta}(s)} \lambda_{n}\right)=\Psi_{0}(\dot{\mathrm{u}}(s))+\frac{\lambda_{n}}{2 n \tau_{n}^{\delta}(s)}\|\dot{\mathrm{u}}(s)\|^{2} \\
& =\tau_{n}^{\delta}(s) \Psi_{n}\left(\frac{\dot{\mathrm{u}}(s)}{\tau_{n}^{\delta}(s)}\right)+\left(\lambda_{n}-1\right) \frac{\|\dot{\mathrm{u}}(s)\|^{2}}{2 n \tau_{n}^{\delta}(s)} .
\end{aligned}
$$

It follows from (6.39) and (6.40) that, for $n$ sufficiently big,

$$
n \tau_{n}^{\delta}(s) \geq \frac{\|\dot{\mathrm{u}}(s)\|}{1+d_{*}\left(-\mathrm{D} \mathcal{E}(\mathrm{t}(s), \mathrm{u}(s)), K^{*}\right)},
$$

hence we deduce that

$$
\frac{\dot{\mathrm{t}}_{n}(s)}{\lambda_{n}} \Psi_{n}\left(\frac{\dot{\mathrm{u}}(s)}{\dot{\mathrm{t}}_{n}(s)} \lambda_{n}\right)-\tau_{n}^{\delta}(s) \Psi_{n}\left(\frac{\dot{\mathrm{u}}(s)}{\tau_{n}^{\delta}(s)}\right) \leq\left(\lambda_{n}-1\right) C \quad \text { for a.a. } s \in(0, S) .
$$

Then, (6.35), and ultimately (6.21), ensue, since $\lambda_{n} \rightarrow 1$.

It remains to verify the integrated inequality (6.23). For this, we apply the Fatou's lemma by checking the validity of conditions (6.22). They can be verified using (6.25) and resorting to the uniform (w.r.t. $s \in(0, S)$ ) estimates $(6.42),(6.44),(6.45)$, and (6.46), as well as to the following estimate

$$
\begin{aligned}
\mathrm{b}_{\Psi_{n}}\left(\tau_{n}^{\delta}(s), \dot{\mathrm{u}}(s),-\mathrm{DE}(\mathrm{t}(s), \mathrm{u}(s))\right) & =\sup \left\{\langle\xi, \dot{\mathrm{u}}(s)\rangle \mid \xi \in K_{n, \delta}^{*}(-\mathrm{DE}(\mathrm{t}(s), \mathrm{u}(s)))\right\} \\
& \leq \sup \left\{\|\dot{\mathrm{u}}(s)\|_{1}\|\xi\|_{\infty} \mid \xi \in K_{n, \delta}^{*}(-\mathrm{DE}(\mathrm{t}(s), \mathrm{u}(s)))\right\} \\
& \leq C\|\dot{\mathrm{u}}(s)\|_{1}\left(\|\mathrm{DE}(\mathrm{t}(s), \mathrm{u}(s))\|_{\infty}+\sqrt{\frac{\delta}{n}}\right),
\end{aligned}
$$

again by the general representation formula (5.22). We then conclude (6.22) by taking into account the boundedness of $\|\dot{\mathrm{u}}(s)\|_{1},\|\mathrm{DE}(\mathrm{t}(s), \mathrm{u}(s))\|_{\infty}$ and sending $n \rightarrow \infty$. Thus, (6.23) is proven.

Step 7: recovery sequence for a general curve u and conclusion of the proof. Now we construct a recovery sequence for a curve with countably many jumps, following the argument from the proof of [12, Thm. 4.2]. Given the jump set $\mathrm{J}_{u}$, fix $\varepsilon>0$, consider a countable set $\left\{t^{i}\right\}_{i \in I} \subseteq \mathrm{J}_{u} \cup\{0, T\}$ (with $t^{i}<t^{i+1}$ for all $i \in I$ ) such that

$$
\operatorname{Jmp}_{\mathrm{p}, \mathcal{E}}\left(u ;[0, T] \backslash\left\{t^{i}\right\}\right)<\varepsilon,
$$

(recall the definition (3.22) of $\mathrm{Jmp}_{\mathrm{p}, \mathcal{E}}$ ), and such that the interval $[0, T]$ can be written as the union of disjoint subintervals

$$
[0, T]=\bigcup_{i \in I} \Sigma^{i} \quad \text { where } \Sigma^{i}=\left[t^{i}, t^{i+1}\right] .
$$

Then, we let $t_{n}^{i}=\mathrm{t}_{n}\left(\mathrm{~s}\left(t^{i}\right)\right)$, and set

$$
\lambda_{n}^{i}:=\frac{t_{n}^{i+1}-t_{n}^{i}}{t^{i+1}-t^{i}}
$$

We define the recovery sequence by

$$
u_{n}(t):=\mathrm{u}\left(\mathrm{t}_{n}^{-1}\left(\lambda_{n}^{i}\left(t-t^{i}\right)+t_{n}^{i}\right)\right) \quad \text { for } t \in \Sigma^{i},
$$


so that

$$
\dot{u}_{n}(t)=\frac{\dot{\mathrm{u}}}{\dot{\mathrm{t}}_{n}}\left(\mathrm{t}_{n}^{-1}\left(\lambda_{n}^{i}\left(t-t^{i}\right)+t_{n}^{i}\right)\right) \lambda_{n}^{i} \quad \text { for } t \in\left(\Sigma^{i}\right)^{\circ} .
$$

We have now that

$$
\begin{aligned}
\int_{0}^{T}( & \left.\Psi_{n}\left(\dot{u}_{n}(t)\right)+\Psi_{n}^{*}\left(-\mathrm{D} \mathcal{E}\left(t, u_{n}(t)\right)\right)\right) \mathrm{d} t \\
= & \sum_{i} \int_{\Sigma^{i}}\left(\Psi_{n}\left(\frac{\dot{\mathrm{u}}}{\dot{\mathrm{t}}_{n}}\left(\mathrm{t}_{n}^{-1}\left(\lambda_{n}^{i}\left(t-t^{i}\right)+t_{n}^{i}\right)\right) \lambda_{n}^{i}\right)\right. \\
& \left.+\Psi_{n}^{*}\left(-\mathrm{D} \mathcal{E}\left(t, \mathrm{u}\left(\mathrm{t}_{n}^{-1}\left(\lambda_{n}^{i}\left(t-t^{i}\right)+t_{n}^{i}\right)\right)\right)\right)\right) \mathrm{d} t \\
= & \sum_{i} \int_{\mathrm{s}\left(t^{i}\right)}^{\mathrm{s}\left(t^{i+1}\right)}\left[\Psi_{n}\left(\frac{\dot{\mathrm{u}}^{(}(s)}{\dot{\mathrm{t}}_{n}(s)} \lambda_{n}^{i}\right)+\Psi_{n}^{*}\left(-\mathrm{D} \mathcal{E}\left(\left(\lambda_{n}^{i}\right)^{-1}\left(\mathrm{t}_{n}(s)-t_{n}^{i}\right)+t^{i}, \mathrm{u}(s)\right)\right)\right] \frac{\dot{\mathrm{t}}_{n}(s)}{\lambda_{n}^{i}} \mathrm{~d} s .
\end{aligned}
$$

Applying estimate (6.21) in every subinterval $\left[\mathrm{s}\left(t^{i}\right), \mathrm{s}\left(t^{i+1}\right)\right]$ and Fatou's lemma (cf. (6.22)) on the whole interval $[0, S]$, we obtain inequality $(6.15)$.

The convergence of the variations again follows by the definition of $u_{n}$. The pointwise convergence $u_{n}(t) \rightarrow u(t)$ for $t \in[0, T] \backslash \mathrm{J}_{u}$ is again trivial. The following calculations show that, by construction, the convergence holds also in the points $\left\{t^{i}\right\} \subseteq \mathrm{J}_{u}$. Indeed,

$$
u_{n}\left(t^{i}\right) \stackrel{(6.48)}{=} \mathrm{u}\left(\mathrm{t}_{n}^{-1}\left(t_{n}^{i}\right)\right)=\mathrm{u}\left(\mathrm{t}_{n}^{-1}\left(\mathrm{t}_{n}\left(\mathrm{~s}\left(t^{i}\right)\right)\right)\right)=\mathrm{u}\left(\mathrm{s}\left(t^{i}\right)\right) \stackrel{(6.12)}{=} u\left(t^{i}\right),
$$

while from (6.47) and the convergence of the variations we have that

$$
\lim _{n \rightarrow \infty}\left|u_{n}(t)-u(t)\right|<\varepsilon \quad \text { for all } t \in \mathbf{J}_{u} \backslash\left\{t^{i}\right\} .
$$

In fact, the recovery sequence $u_{n}$ has a hidden dependence on $\varepsilon$. Then taking $\varepsilon=n^{-1}$ we define a new recovery sequence, that we keep labeling $u_{n}$, and sending $n \rightarrow \infty$ ( $\varepsilon$ to zero) we conclude.

This finishes the proof of Theorem 5.9.

Remark 6.2 Let us briefly outline how the proof of Theorem 5.9 carries over to the case in which the dissipation potentials are given by (5.37). Clearly, it is sufficient to comment on the changes in the calculations carried out throughout Step $6 b$, starting from the Euler-Lagrange equation (5.29). In the case

$$
\Psi_{n}(v)=A\|v\|_{1}+\frac{1}{p^{p-1}}\|v\|^{p}, \quad \text { with } \Psi_{n}^{*}(\xi)=\frac{n}{p^{\prime}} d_{*}\left(\xi, K^{*}\right)^{p^{\prime}},
$$

and $p^{\prime}=\frac{p}{p-1}$ the conjugate exponent of $p,(5.29)$ becomes

$$
\left(1-\frac{1}{p}\right) \frac{1}{n^{p-1}}\left\|\frac{\dot{\mathrm{u}}(s)}{\tau_{n}^{\delta}(s)}\right\|^{2}+\frac{n}{p^{\prime}} d_{*}\left(-\mathrm{D} \mathcal{E}(\mathrm{t}(s), \mathrm{u}(s)), K^{*}\right)^{p^{\prime}}+\delta=0 \quad \text { for a.a. } s \in(0, S),
$$

whence we deduce that

$$
\tau_{n}^{\delta}(s)=\frac{1}{n} \frac{\|\dot{\mathrm{u}}(s)\|}{\left(d_{*}\left(-\mathrm{D} \mathcal{E}(\mathrm{t}(s), \mathrm{u}(s)), K^{*}\right)^{p^{\prime}}+2 \frac{\delta}{n}\right)^{1 / p}} \quad \text { for a.a. } s \in(0, S) .
$$

We now write the analogue of (6.41), taking into account formulae (6.49), and prove with straightforward algebra that 


$$
\limsup _{n \rightarrow \infty} \mathrm{b}_{\Psi_{n}}^{\delta}\left(\tau_{n}^{\delta}(s), \dot{\mathrm{u}}(s),-\mathrm{D} \mathcal{E}(\mathrm{t}(s), \mathrm{u}(s))\right) \leq \mathrm{p}(\dot{\mathrm{t}}(s), \dot{\mathrm{u}}(s),-\mathrm{D} \mathcal{E}(\mathrm{t}(s), \mathrm{u}(s))) .
$$

All the remaining calculations in Step $6 b$ extend to the case of (5.37).

Acknowledgements We would like to thank Mark Peletier for suggesting this problem to us and for his support. We are deeply grateful to Giuseppe Savaré for several enlightening discussions, in particular on the results of Sect. 4.

\section{References}

1. Adams, S., Dirr, N., Peletier, M.A., Zimmer, J.: From a large-deviations principle to the Wasserstein gradient flow: a new micro-macro passage. Commun. Math. Phys. 307(3), 791-815 (2011)

2. Adams, S., Drri, N., Peletier, M., Zimmer, J.:: Large deviations and gradient flows. J. Philos. Trans. R. Soc. Lond. Ser. A Math. Phys. Eng. Sci. 371(2005), 20120341 (2013)

3. Ambrosio, L., Fusco, N., Pallara, D.: Functions of Bounded Variation and Free Discontinuity Problems. Oxford University Press, Oxford (2005)

4. Attouch, H.: Variational Convergence for Functions and Operators. Applicable Mathematics Series. Pitman (Advanced Publishing Program), Boston, MA (1984)

5. Basinski, Z.S.: Thermally activated glide in face-centred cubic metals and its application to the theory of strain hardening. Philos. Mag. 4, 393-432 (1959)

6. Buliga, M., de Saxcé, G., Vallée, C.: Existence and construction of bipotentials for graphs of multivalued laws. J. Convex Anal. 15(1), 87-104 (2008)

7. Brézis, H., Ekeland, I.: Un principe variationnel associé à certaines équations paraboliques. Le cas dépendant du temps. C. R. Acad. Sci. Paris Sér. A-B 282((20):Ai), A1197-A1198 (1976)

8. Brézis, H., Ekeland, I.: Un principe variationnel associé à certaines équations paraboliques. Le cas indépendant du temps. C. R. Acad. Sci. Paris Sér. A-B 282((17):Aii), A971-A974 (1976)

9. Becker, R.: Über die plasticität amorpher und kristalliner fester körper. Phys. Z. 26, 919-925 (1925)

10. Berglund, N.: Kramers' law: validity, derivations and generalisations. Markov Process. Relat. Fields 19(3), 459-490 (2013)

11. Babadjian, J.-F., Francfort, G., Mora, M.G.: Quasistatic evolution in non-associative plasticity-the cap model. SIAM J. Math. Anal. 44, 245-292 (2012)

12. Bonaschi, G.A., Peletier, M.A.: Quadratic and rate-independent limits for a large-deviations functional. Contin. Mech. Thermodyn. 28, 1191-1219 (2016)

13. Braides, A.: Local Minimization, Variational Evolution and $\Gamma$-convergence, Volume 2094 of Lecture Notes in Mathematics. Springer, Cham (2014)

14. Crismale, V., Lazzaroni, G.: Viscous approximation of quasistatic evolutions for a coupled elastoplasticdamage model. Calc. Var. Partial Differ. Equ. 55(1), 17 (2016)

15. Colli, P.: On some doubly nonlinear evolution equations in Banach spaces. Jpn. J. Indust. Appl. Math. 9(2), 181-203 (1992)

16. Colli, P., Visintin, A.: On a class of doubly nonlinear evolution equations. Commun. Partial Differ. Equ. 15(5), 737-756 (1990)

17. Dal Maso, G., DeSimone, A., Solombrino, F.: Quasistatic evolution for cam-clay plasticity: a weak formulation via viscoplastic regularization and time rescaling. Calc. Var. Partial Differ. Equ. 40, 125-181 (2011)

18. Dal Maso, G., DeSimone, A., Mora, M.G., Morini, M.: A vanishing viscosity approach to quasistatic evolution in plasticity with softening. Arch. Ration. Mech. Anal. 189(3), 469-544 (2008)

19. Dal Maso, G., Toader, R.: A model for the quasi-static growth of brittle fractures: existence and approximation results. Arch. Ration. Mech. Anal. 162(2), 101-135 (2002)

20. Efendiev, M., Mielke, A.: On the rate-independent limit of systems with dry friction and small viscosity. J. Convex Anal. 13(1), 151-167 (2006)

21. Feng, J., Kurtz, T.G.: Large Deviations for Stochastic Processes, Volume 131 of Mathematical Surveys and Monographs. American Mathematical Society, Providence, RI (2006)

22. Francfort, G.A., Marigo, J.-J.: Revisiting brittle fracture as an energy minimization problem. J. Mech. Phys. Solids 46(8), 1319-1342 (1998)

23. Ghoussoub, N.: Self-Dual Partial Differential Systems and Their Variational Principles. Springer Monographs in Mathematics. Springer, New York (2009)

24. Ioffe, A.D.: On lower semicontinuity of integral functionals. I. SIAM J. Control Optim. 15(4), 521-538 (1977) 
25. Krausz, A.S., Eyring, H.: Deformation Kinetics. Wiley, New York (1975)

26. Knees, D., Mielke, A., Zanini, C.: On the inviscid limit of a model for crack propagation. Math. Models Methods Appl. Sci. 18(9), 1529-1569 (2008)

27. Kramers, H.A.: Brownian motion in a field of force and the diffusion model of chemical reactions. Physica 7(4), 284-304 (1940)

28. Knees, D., Rossi, R., Zanini, C.: A vanishing viscosity approach to a rate-independent damage model. Math. Models Methods Appl. Sci. 23(4), 565-616 (2013)

29. Liero, M., Mielke, A., Peletier, M.A., Renger, D.R.M.: On microscopic origins of generalized gradient structures. Discrete Contin. Dyn. Syst. Ser. S 10(1), 1-35 (2017)

30. Lazzaroni, G., Toader, R.: A model for crack propagation based on viscous approximation. Math. Models Methods Appl. Sci. 21(10), 2019-2047 (2011)

31. Mielke, A.: Energetic formulation of multiplicative elasto-plasticity using dissipation distances. Cont. Mech. Thermodyn. 15, 351-382 (2003)

32. Mielke, A.: Evolution in rate-independent systems (Ch.6). In: Dafermos, C.M., Feireisl, E. (eds.) Handbook of Differential Equations, Evolutionary Equations, vol. 2, pp. 461-559. Elsevier, Amsterdam (2005)

33. Mielke, A.: Differential, energetic, and metric formulations for rate-independent processes. In: Nonlinear PDE's and Applications, Volume 2028 of Lecture Notes in Mathematics, pp. 87-170. Springer, Heidelberg (2011)

34. Mielke, A.: On evolutionary $\Gamma$-convergence for gradient systems. In: Macroscopic and Large Scale Phenomena: Coarse Graining. Mean Field Limits and Ergodicity, Volume 3 of Lecture Notes in Applied Mathematics and Mechanics, pp. 187-249. Springer, Cham (2016)

35. Mainik, A., Mielke, A.: Existence results for energetic models for rate-independent systems. Calc. Var. Partial Differ. Equ. 22, 73-99 (2005)

36. Mach Nguyet, M.: BV solutions constructed using the epsilon-neighborhood method. ESAIM Control Optim. Calc. Var. 22(1), 188-207 (2016)

37. Moreau, J.-J.: Bounded variation in time. In: Moreau, J.-J., Panagiotopoulos, P.D., Strang, G. (eds.) Topics in Nonsmooth Mechanics, pp. 1-74. Birkhäuser, Basel (1988)

38. Mielke, A., Peletier, M.A., Renger, D.R.M.: On the relation between gradient flows and the large-deviation principle, with applications to Markov chains and diffusion. Potential Anal. 41(4), 1293-1327 (2014)

39. Mielke, A., Roubíček, T.: Rate-Independent Systems. Theory and Application, Volume 193 of Applied Mathematical Sciences. Springer, New York (2015)

40. Mielke, A., Roubíček, T., Stefanelli, U.: $\Gamma$-limits and relaxations for rate-independent evolutionary problems. Calc. Var. Partial Differ. Equ. 31, 387-416 (2008)

41. Mielke, A., Rossi, R., Savaré, G.: BV solutions and viscosity approximations of rate-independent systems. ESAIM Control Optim. Calc. Var. 18(1), 36-80 (2012)

42. Mielke, A., Rossi, R., Savaré, G.: Balanced viscosity (BV) solutions to infinite-dimensional rateindependent systems. J. Eur. Math. Soc. 18(9), 2107-2165 (2016)

43. Mielke, A., Theil, F.: A mathematical model for rate-independent phase transformations with hysteresis. In: Alber, H.-D., Balean, R.M., Farwig, R. (eds.) Proceedings of the Workshop on Models of Continuum Mechanics in Analysis and Engineering, pp. 117-129, Shaker, Aachen (1999)

44. Mielke, A., Theil, F.: On rate-independent hysteresis models. NoDEA Nonlinear Differ. Equ. Appl. 11(2), 151-189 (2004)

45. Nayroles, B.: Deux théorèmes de minimum pour certains systèmes dissipatifs. C. R. Acad. Sci. Paris Sér. A-B 282(17):Aiv, A1035-A1038 (1976)

46. Negri, M.: Quasi-static rate-independent evolutions: characterization, existence, approximation and application to fracture mechanics. ESAIM Control Optim. Calc. Var. 20(4), 983-1008 (2014)

47. Orowan, E.: Problems of plastic gliding. Proc. Phys. Soc. 52, 8-22 (1940)

48. Rossi, R., Savaré, G.: A characterization of energetic and BV solutions to one-dimensional rateindependent systems. Discrete Contin. Dyn. Syst. Ser. S 6(1), 167-191 (2013)

49. Serfaty, S.: Gamma-convergence of gradient flows on Hilbert and metric spaces and applications. Discrete Contin. Dyn. Syst. 31(4), 1427-1451 (2011)

50. Sandier, E., Serfaty, S.: Gamma-convergence of gradient flows with applications to Ginzburg-Landau. Commun. Pure Appl. Math. 57(12), 1627-1672 (2004)

51. Stefanelli, U.: The Brézis-Ekeland principle for doubly nonlinear equations. SIAM J. Control Optim. 47(3), 1615-1642 (2008)

52. Toader, R., Zanini, C.: An artificial viscosity approach to quasistatic crack growth. Boll. Unione Mat. Ital. 9(2), 1-35 (2009)

53. Valadier, M.: Young measures. In: Cellina, A. (ed.) Methods of nonconvex analysis (Varenna, 1989), pp. 152-188. Springer, Berlin (1990) 
54. Visintin, A.: Variational formulation and structural stability of monotone equations. Calc. Var. Partial Differ. Equ. 47(1-2), 273-317 (2013)

Publisher's Note Springer Nature remains neutral with regard to jurisdictional claims in published maps and institutional affiliations. 